$\frac{34}{10} \cdot 2996(2)$

SSCL-484

Superconducting Super Collider Laboratory

岁

The SSC Beam Scraper System

M. Maslov, N. Mokhov, and L. Yazynin

June 1991 


\title{
The SSC Beam Scraper System
}

\author{
M.A. Maslov, N.V. Mokhov, and I.A. Yazynin \\ Accelerator Division \\ Superconducting Super Collider Laboratory* \\ 2550 Beckleymeade Ave. \\ Dallas, TX 75237 \\ and \\ Institute for High Energy Physics \\ Protvino, U.S.S.R. \\ June 1991
}

\section{DISCLAIMER}

\begin{abstract}
This report was prepared as an account of work sponsored by an agency of the United States Government. Neither the United States Government nor any agency thereof, nor any of their employees, makes any warranty, express or implied, or assumes any legal liability or responsibility for the accuracy, completeness, or usefulness of any information, apparatus, product, or process disclosed, or represents that its use would not infringe privately owned rights. Reference herein to any specific commercial product, process, or service by trade name, trademark, manufacturer, or otherwise does not necessarily constitute or imply its endorsement, recommendation, or favoring by the United States Government or any agency thereof. The views and opinions of authors expressed herein do not necessarily state or reflect those of the United States Government or any agency thereof.
\end{abstract}

\footnotetext{
* Operated by the Universities Research Association, Inc., for the U.S. Department of Energy under Contract No. DE-AC02-89ER40486.
} 


\section{CONTENTS}

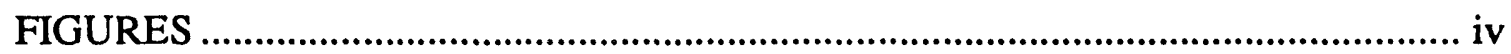

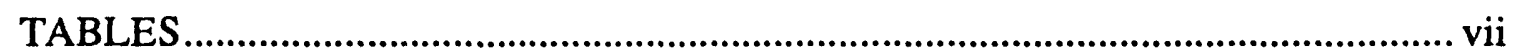

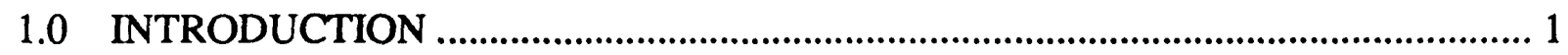

2.0 BEAM RELATED PARAMETERS …....................................................... 1

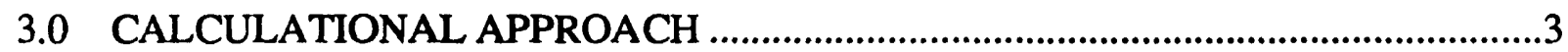

4.0 BEAM LOSS IN THE MACHINE................................................................ 4

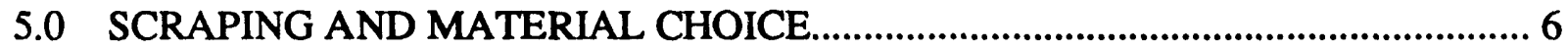

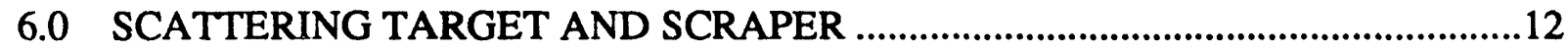

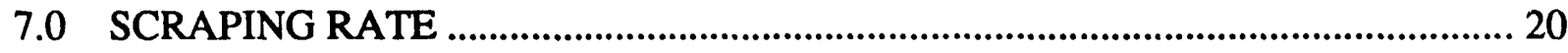

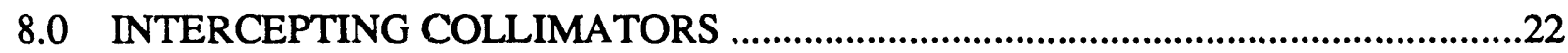

9.0 SCRAPER SYSTEM IN THE EAST CLUSTER .............................................. 23

10.0 SCRAPER: COOLING, SHIELDING, RADIOACTIVATION ............................. 39

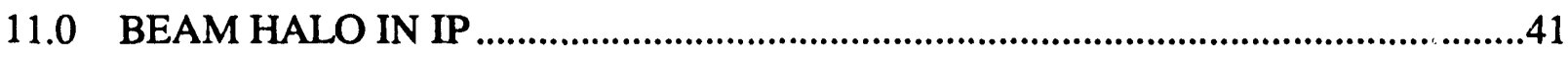

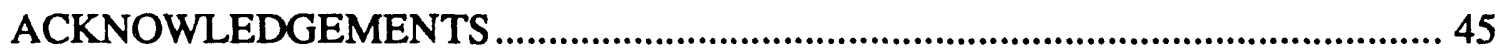

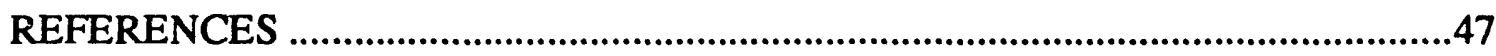




\section{FIGURES}

1. Beam halo rate due to $\mathrm{pp}$-collisions and beam-gas scattering versus distance from the beam centerline (from Reference 15) ............................................................... 5

2. Transverse distribution of protons from pp-diffraction for two values

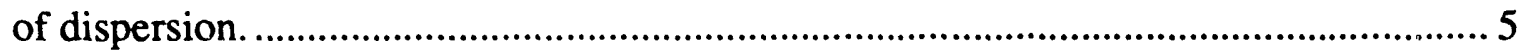

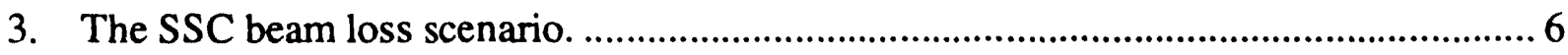

4. Dynamics of beam halo-scraper interaction: 1) beam lead up to the scraper (solid); 2) large amplitude protons (long dash); 3) off-momentum protons (short dash) ................................................................................................ 7

5. Proton impact parameter at the scraper versus transverse drift speed............................ 8

6. Comparison of the maximum energy deposition density as a function of longitudinal distance for tungsten, copper, and graphite scrapers. ......................... 9

7. Absorption efficiency for scrapers made of various materials as a function of the scraper length

8. Total beam loss in the SSC lattice per one scraped proton versus transverse drift speed

9. Total beam loss in the SSC lattice per one proton scraped by tungsten or copper scraper at injection and full energy as a function of the impact parameter.

10. Schematic of scraper, target and radiation shielding: a) longitudinal view; b) transverse cross section.

11. Comparison of proton impact parameter distribution at the scraper upstream end for the different scraping schemes with and without a scattering larget. Note effectiveness of target in spreading impact distribution.

12. Maximum energy deposition per scraped proton $(20 \mathrm{TeV})$ as a function of longitudinal distance in copper scraper: with no target, with 1-mm tungsten target, and with 5-mm silicon bent crystal at the front of the scraper.

Note dramatic decrease of energy deposition for first bin.

13. Maximum energy deposition per scraped proton $(2 \mathrm{TeV})$ as a function of longitudinal distance in copper scraper: with no target and with 1-mm tungsten target.

14. Absorption efficiency of copper scraper as a function of scraper length for different scraping modes at $20 \mathrm{TeV}$.

15. Absorption efficiency of copper scraper as a function of scraper length for different scraping modes at $2 \mathrm{TeV}$.

16. Amplitude distribution of outscattered protons to be lost on the machine aperture for scraping at $20 \mathrm{TeV}$ with and without target.

17. Momentum distribution of outscattered protons for scraping at $20 \mathrm{TeV}$ with and without target. 
18. Proton transverse displacement distribution on target (short dash) and scraper (solid) at $0.3 \mathrm{~mm} / \mathrm{s}$ transverse drift speed. Target inner edge is positioned at $0.8 \mathrm{~mm}$ and the scraper edge at $0.85 \mathrm{~mm}$.

19. Proton transverse displacement distribution on target (short dash) and scraper (solid) at $1.5 \mathrm{~mm} / \mathrm{s}$ transverse drift speed. Target inner edge is positioned at $0.8 \mathrm{~mm}$ and the scraper edge at $0.85 \mathrm{~mm}$.

20. Probability of target crossing and number of turns after the first pass through the target as a function of number of turns.

21. Total beam loss in the SSC lattice per one proton scraped by tungsten scraper versus scraper alignment angle.

22. Efficiency of interception by a collimator positioned at $n \sigma$ from the beam axis of protons leaked out with angle $\delta x^{\prime}$ of the scraper, positioned at $m \sigma$

23. Total beam loss in the SSC lattice per one 20-TeV proton scraped by tungsten or copper scraper versus $\beta$-function at the scraper position.

24. Total beam loss in the SSC lattice per one $20-\mathrm{TeV}$ proton scraped by copper scraper with and without tungsten target as a function of the accelerator admittance.

25. Maximum energy deposition (per one scraped proton) in the superconducting coils of two dipoles $7 \mathrm{~m}$ downstream of the $3 \mathrm{~m}$ copper scraper (with a target) with additional collimator with ID $=34 \mathrm{~mm}$, length $=3 \mathrm{~m}$ (run 1) and $\mathrm{ID}=20 \mathrm{~mm}$, length $=5 \mathrm{~m}$ (run 2) just before the first dipole.

26. Lattice functions and schematic magnet layout for an east utility straight section scraper system.

27. Trajectories of protons elastically scattered out of scraper.

28. Trajectories of protons which exit the scraper with various momentum change in the east utility straight section.

29. Trajectories of protons which exit the scraper with various momentum change in the east utility straight section, with a horizontal dogleg out of the ring center.

30. Trajectories of protons which exit the scraper with various momentum change in the east utility straight section, with a horizontal dogleg to the ring center.

31. Trajectories of protons which exit the scraper with various momentum change in the east utility straight section, with a vertical dogleg.

32. Horizontal and vertical distributions of protons to be lost on the machine aperture, at collimator $\mathrm{Cl}$ position

33. Angular distributions of protons to be lost on the machine aperture, at collimator $\mathrm{Cl}$ position

34. Vertical plane phase space portrait for protons to be lost in the machine, at collimator $\mathrm{C} 1 / \mathrm{C} 2$ position. 
35. Phase space portrait in horizontal plane of protons to be lost in the machine, at collimators $\mathrm{C} 1 / \mathrm{C} 2$ and $\mathrm{C} 3 / \mathrm{C} 4$ positions.

36. Phase space portrait in horizontal plane of protons to be lost in the machine, at collimators $\mathrm{C} 1 / \mathrm{C} 2$ and $\mathrm{C} 3 / \mathrm{C} 4$ positions, with collimator $\mathrm{C} 1$ aperture equal to $\pm 5 \mathrm{~mm}$.

37. Total beam loss in the SSC lattice for $10^{10}$ protons per second scraping rate at scraper SCR-X (the first $15 \mathrm{~min}$ at full energy) versus $\mathrm{C} 1 / \mathrm{C} 2$ aperture.

38. Total beam loss in the SSC lattice for $10^{10}$ protons per second scraping rate at vertical scraper SCR-Y (the first 15 min at full energy) versus C1/C2 aperture. ..... 34

39. Beam loss density distribution in the east utility downstream of the scrapers for a scraping rate of $10^{10}$ protons per second.

40. Beam loss density distribution in the east utility downstrean of the scrapers for a scraping rate of $10^{10}$ protons per second, with the bump magnet system

41. Beam loss density distribution in the east utility downstream of the scrapers for a scraping rate of $10^{10}$ protons per second, with the bump magnet system and with the additional collimators.

42. Beam loss density distribution in the dispersion suppressors.

43. Maximum energy deposition density in the superconducting coils of the dispersion suppressor elements as a function of distance from collimator C3/C4 ....... 38

44. Power deposited per $1 \mathrm{~m}$ of the dispersion suppressors for $10^{10} \mathrm{p} / \mathrm{s}$ scraping rate.

45. Longitudinal distribution of maximum energy deposition density in the copper scraper for chosen parameters of the scraper system.

46. Lateral distribution of energy deposition in the copper scraper at shower maximum and averaged over $120 \mathrm{~cm}$ of length.

47. Cumulative distribution of energy absorbed in the copper scraper..

48. Phase space portrait of the outscattered protons at the entrance to the scraper system

49. Normalized phase space portrait of protons outscattered from the scraper.

50. Distribution of halo particles with no scraping, with single scraper, with target/scraper couple, and with collimators $\mathrm{Cl}$ and $\mathrm{C} 3$ installed additionally at $7 \sigma$ from the beam centerline (histogram). 


\section{TABLES}

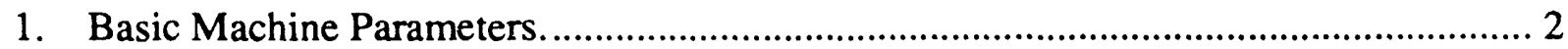

2. SSC Beam Parameters: Dispersion, Maximal $\beta$-Function, and r.m.s Beam Sizes........ 2

3. Definition of Maximum Beam Excursion at $N \sigma$................................................. 3

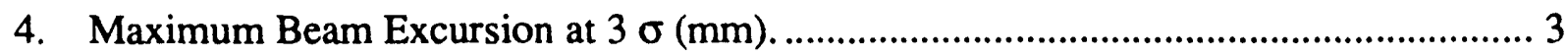

5. Maximum Beam Excursion at $6 \sigma(\mathrm{mm})$........................................................... 3

6. Energy Deposition $\mathrm{Q}$ and Temperature $\mathrm{T}_{\max }$ limits..............................................8 8

7. Number of Outscattered Protons (\%) per 1 Incident Proton and Yield Angle. ........... 16

8. Maximum Energy Deposition in the Scraper and Number of Protons in the Fast Spill Causing the Material Destruction..................................................................21

9. Maximum Scraping Rate in the Collider to Design the Scraper System.................... 21

10. Scraper System Parameters: Target Scrapers and Collimators...................................26

11. Scraper System Parameters: Dogleg Magnets. .........................................................27

12. Scraper System Parameters: Lead Up Magnets. ...................................................... 36

13. Maximum Energy Deposition Rate $q$, Deposited Power $P$, and Annual Dose $D$ in the Scraper System for "Peak" $\left(1.4 \times 10^{10} \mathrm{p} / \mathrm{s}\right)$ and "Steady State" $\left(1.6 \times 10^{8} \mathrm{p} / \mathrm{s}\right)$ Scraping Rates 


\subsection{INTRODUCTION}

In the Superconducting Super Collider (SSC), ${ }^{1}$ as in any other accelerator, the formation of a beam halo due to a variety of reasons is unavoidable. Proton scattering in pp-collisions and in beam-gas interactions and the diffusion of particles due to various non-linear phenomena out of the beam-core-all result in emittance growth and eventually in beam loss in the lattice. The radiation effects in the lattice elements, with emphasis on the superconducting magnets, and some possible protective measures have been analyzed in detail in an earliei report. ${ }^{2}$ Another consequence of the beam halo is the increase of the beam size at interaction points (IP) and of higher background rate at the experimental setups. Therefore, a very efficient beam scraper system must be designed and installed in order to provide reliable operation of the superconducting machine, to sustain favorable experimental conditions, and to have minimal impact of radiation on equipment, personnel, and the environment. A preliminary investigation on such a system for the SSC is described elsewhere. ${ }^{1-4}$ We note that there is experience on this subject at both Fermilab ${ }^{5}$ and CERN. ${ }^{6}$

In this paper we present the results of a full-scale study of a beam scraping system that is designed to guarantee reliable operation of the SSC throughout the whole cycle and for minimum background for experiments at the interaction regions. The machine aperture limits and beam loss formation are analyzed. Simulation programs and a calculational model are described. The physics of beam scraping is explored, and measures to increase significantly the system efficiency are determined. A tolerable scraping rate, taking into account scraper material integrity, quench limits in downstream superconducting magnets, radiation shielding requirements, and minimal beam halo levels at the IPs are also determined. Finally, a complete multi-component scraper system in the SS: East Cluster is proposed.

Throughout the paper we define a scraper as a primary absorber consisting of precise movable jaws that have a flat inner edge along the circulating beam and which may be forced to touch the beam halo in horizontal or vertical planes. Secondary absorbers-collimators-are destined to intercept outscattered protons and other particles produced in scraper material. All these are surrounded with a radiation shielding.

\subsection{BEAM RELATED PARAMETERS}

Basic SSC parameters ${ }^{1}$ related to the considered problem are listed in Tables 1 and 2. The number of protons circulating in each of the Collider rings is $1.3 \times 10^{14}$. The dipole aperture is 50 $\mathrm{mm}$, and the beam pipe diameter is $40 \mathrm{~mm}$. Dispersion and $\beta$-functions for the East Utility 
straight section given in Table 2 have been calculated with SYNCH by Al Garren ${ }^{7}$ within a new lattice designed to be compatible with our scraper system requirements.

Table 1. Basic Machine Parameters.

\begin{tabular}{|c|c|c|}
\hline & Injection & Collisions \\
\hline Energy (TeV) & 2 & 20 \\
\hline Gamma & 2131.6 & 21316.0 \\
\hline Bucket half height $x_{p}=\Delta p / p, 10^{-4}$ & 2 & 0.5 \\
\hline Emittance $(\pi \mathrm{mm}-\mathrm{mrad}), 10^{-4}$ & 4.7 & 0.47 \\
\hline Normalized emittance $\varepsilon$ ( $\pi \mathrm{mm}$-mrad) & 1 & 1 \\
\hline Closed orbit peak amplitude $A(\mathrm{~mm})$ arcs: & 2 & 2 \\
\hline other. & 1 & 1 \\
\hline
\end{tabular}

Table 2. SSC Beam Parameters: Dispersion, Maximal $\beta$-Function, and r.m.s Beam Sizes.

\begin{tabular}{lccccccc}
\hline & $\begin{array}{l}\eta_{x} \\
(\mathrm{~m})\end{array}$ & $\begin{array}{l}\beta_{x} \max \\
(\mathrm{m})\end{array}$ & $\begin{array}{l}\beta_{y} \max \\
(\mathrm{m})\end{array}$ & $\begin{array}{l}\sigma_{x} \\
(\mathrm{~mm})\end{array}$ & $\begin{array}{l}\sigma_{y} \\
(\mathrm{~mm})\end{array}$ & $\begin{array}{l}\sigma_{x} \\
(\mathrm{~mm})\end{array}$ & $\begin{array}{l}\sigma_{y} \\
(\mathrm{~mm})\end{array}$ \\
\hline Arcs & & & & \multicolumn{2}{c}{ Injection } & \multicolumn{2}{c}{ Collisions } \\
IR: IP & 1.82 & 305. & 305. & 0.38 & 0.38 & 0.12 & 0.12 \\
IR:QL2(2 TeV) & 0. & 0.5 & 0.5 & .05 & .01 & .005 & .005 \\
IR:QL2(20 TeV) & 0. & 600. & 600. & 0.53 & 0.53 & & \\
Util: SCR & 0. & 7752. & 7752. & & & 0.61 & 0.61 \\
Util: C1/C2 & -4. & 1200. & 480. & 0.75 & 0.48 & 0.24 & 0.15 \\
Util: C3/C4 & -4. & 360. & 500. & 0.41 & 0.48 & 0.13 & 0.15 \\
\hline \hline
\end{tabular}

The estimated beam excursions at different SSC regions defined in Table 3 are given in Tables 4 and 5.

The nominal position of a scraper at full energy is supposed to be close to $6 \sigma$, where $\sigma$ is the r.m.s. beam size. These results give a first guide to choosing the collimator positions throughout the machine. 
Table 3. Definition of Maximum Beam Excursion at $N \sigma$.

\begin{tabular}{lll}
\hline & $\Delta x$ & $\Delta y$ \\
\hline Arcs & $N \sigma+A+S+x_{p}$ & $N \sigma+A$ \\
IR: QL2 & $N \sigma+A+\operatorname{sep}$ & $N \sigma+A+c r o$ \\
Utility & $N \sigma+A$ & $N \sigma+A$ \\
\hline
\end{tabular}

$\mathrm{S}=1.4 \mathrm{~mm}$ - half horizontal sagitta.

sep $=10 \sigma / 2$ - separation, for QL2 sep $=3 \mathrm{~mm}$.

Crossing: for $\mathrm{QL2}$ cro $=5.5 \mathrm{~mm}$, due to crossing angle $=150 \mu \mathrm{rad}$.

Table 4. Maximum Beam Excursion at $3 \sigma(\mathrm{mm})$.

\begin{tabular}{lllll}
\hline & \multicolumn{2}{c}{ Injection } & \multicolumn{2}{c}{ Collisions } \\
& $\Delta x$ & $\Delta y$ & $\Delta x$ & $\Delta y$ \\
\hline Arcs & 5.0 & 3.2 & 3.9 & 2.4 \\
IR: QL2 & 5.3 & 8.1 & 5.8 & 8.3 \\
Util: SCR & 3.6 & 2.5 & 1.9 & 1.5 \\
Util: C1/C2 & 3.0 & 2.5 & 1.6 & 1.5 \\
\hline \hline
\end{tabular}

Table 5. Maximum Eeam Excursion at $6 \sigma(\mathrm{mm})$.

\begin{tabular}{llccr}
\hline & \multicolumn{2}{c}{ Injection } & \multicolumn{2}{c}{ Collisions } \\
& $\Delta x$ & $\Delta y$ & $\Delta x$ & $\Delta y$ \\
\hline Arcs & 6.1 & 4.3 & 4.2 & 2.7 \\
IR: QL2 & 6.9 & 9.7 & 7.6 & 10.1 \\
Util: SCR & 5.4 & 3.9 & 2.7 & 1.9 \\
Util: Ci/C2 & 4.2 & 3.9 & 2.0 & 1.9 \\
\hline \hline
\end{tabular}

\subsection{CALCULATIONAL APPROACH}

The techniques ${ }^{8-12}$ used in simulating the beam halo formation in the machine, the scraping process, the beam loss distribution in the SSC lattice, and the corresponding radiation effects, and then for designing an optimal beam scraping system, are the same as those described in a previous paper $^{2}$ and used at the Tevatron ${ }^{13}$ and at UNK. ${ }^{14}$ We adopt a four-step approach: calculation of beam halo production, simulation of interactions of the halo particles with a target'scraper system, multi-turn tracking of the outscattered protons through the lattice, and finally a full-scale 
simulation of hadronic-electromagnetic cascades in the lattice elements irradiated by lost outscattered protons and secondary debris.

The multi-turn tracking of protons with $p>0.7 p_{O}$ is done using the linear transfer matrix for each dipole, quadrupole, and drift space at about $3 \mathrm{~km}$ downstream of the initial source (target, scraper, or collimator) and then using a single matrix for the rest of the lattice. Information on the protons lost throughout the machine and those that form a beam halo at the IPs is stored in a file for further analysis. The variables recorded are as follows: longitudinal coordinate $s$; transverse coordinates $x, x^{\prime}, y, y^{\prime} ;$ momentum off-set $\Delta p=p_{O}-p$; and statistical weight $w$.

Inclusive simulation of particle interactions in matter in the $0.1 \mathrm{MeV}-20 \mathrm{TeV}$ energy range is done in this work with the newest version of the MARS system, ${ }^{12}$ MARS 12 code. Proton leakage, three-dimensional distributions of energy deposition, and particle fluence are calculated where all the details of element geometry and magnetic field in each region of interest are taken into account up to about $1 \mathrm{~km}$ length.

Typical statistics are 20,000 particles for the scraping and tracking studies and 30,000 cascades for the energy deposition exploration in the scraper system components and in the superconducting magnets.

\subsection{BEAM LOSS IN THE MACHINE}

As mentioned in the introduction, there are several reasons for beam halo creation and consequent beam loss in the machine. Experience at existing accelerators is that beam loss with a complex space-time structure occurs through the whole machine cycle. Without special protective measures, such loss can easily result in exceeding a quench level in superconducting magnets ${ }^{2}$ and in unacceptably large beam size and background in collider experiments.

The quantitative influence of all the non-linear phenomena on the particle transverse diffusion cannot be precisely predicted theoretically. ${ }^{6}$ The beam loss rate in the SSC is about $10^{8} \mathrm{p} / \mathrm{s}$ for beam-gas scattering 2 and $10^{8} \mathrm{p} / \mathrm{s}$ for each IR at $10^{33} \mathrm{~cm}^{-2} \mathrm{~s}^{-1}$ luminosity. A corresponding beam halo rate at a few beam- $\sigma$ level is calculated to be $2-4 \times 10^{7} \mathrm{p} / \mathrm{s}^{15}$ (Figures 1 and 2). Various instabilities, injection and other errors, RF-noise, magnetic imperfections, ripple of power supplies, etc., can easily raise this number by one order of magnitude or even more.

Figure 3 shows the SSC beam loss scenario based on Tevatron experience and design report ${ }^{1}$ assumptions.

A peak at the beginning of acceleration, which exists for all machines, is due to protons not captured into the stable acceleration region during the injection stage. One can expect a modest peak in the transition region. As for CDF experience, a fe w percent of the beam can be lost at the beginning of the collider run during the beam cleaning procedure. 


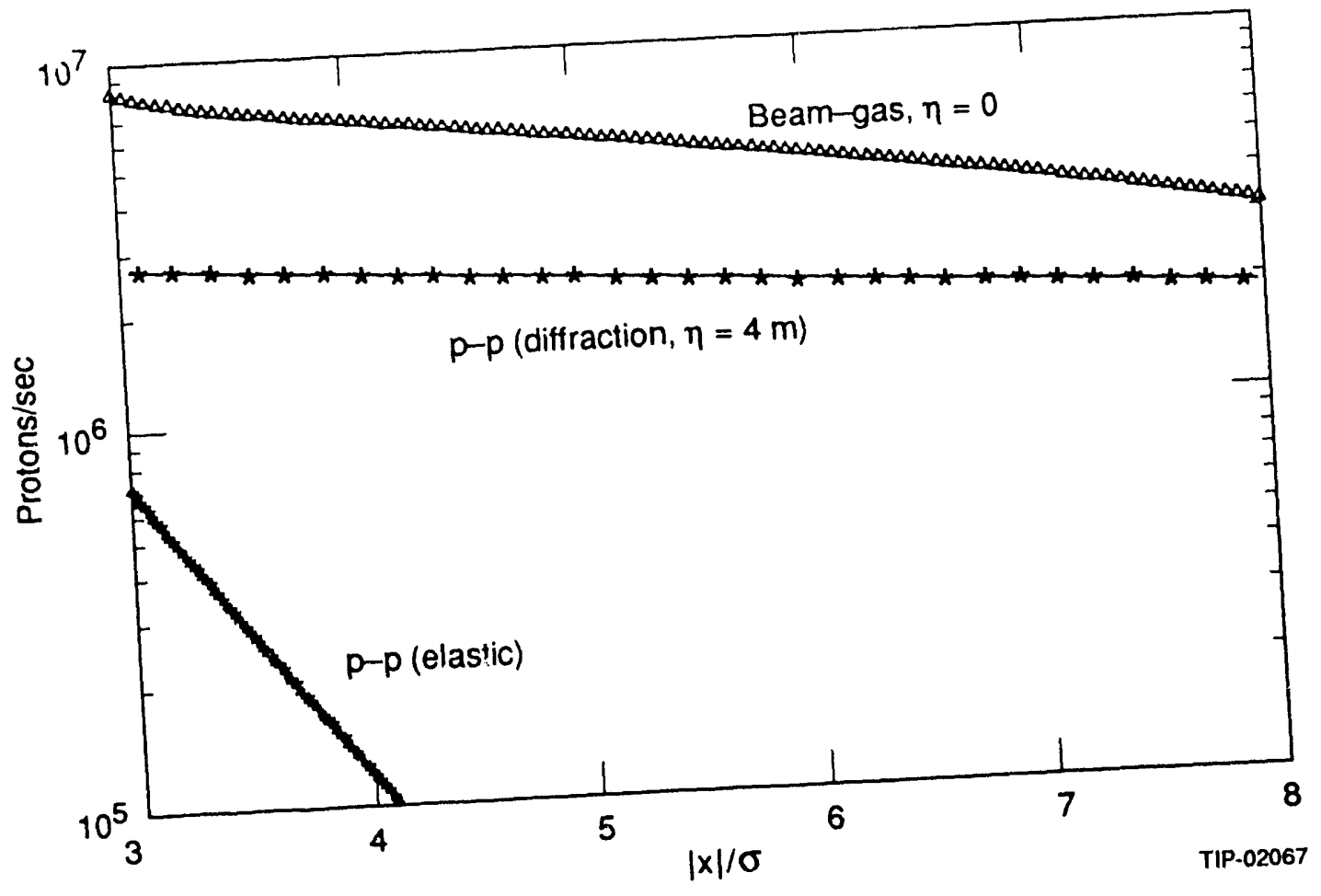

Figure 1. Beam Halo Rate Due to pp-Collisions and Beam-Gas Scattering Versus Distance from the Beam Centerline (from Reference 15).

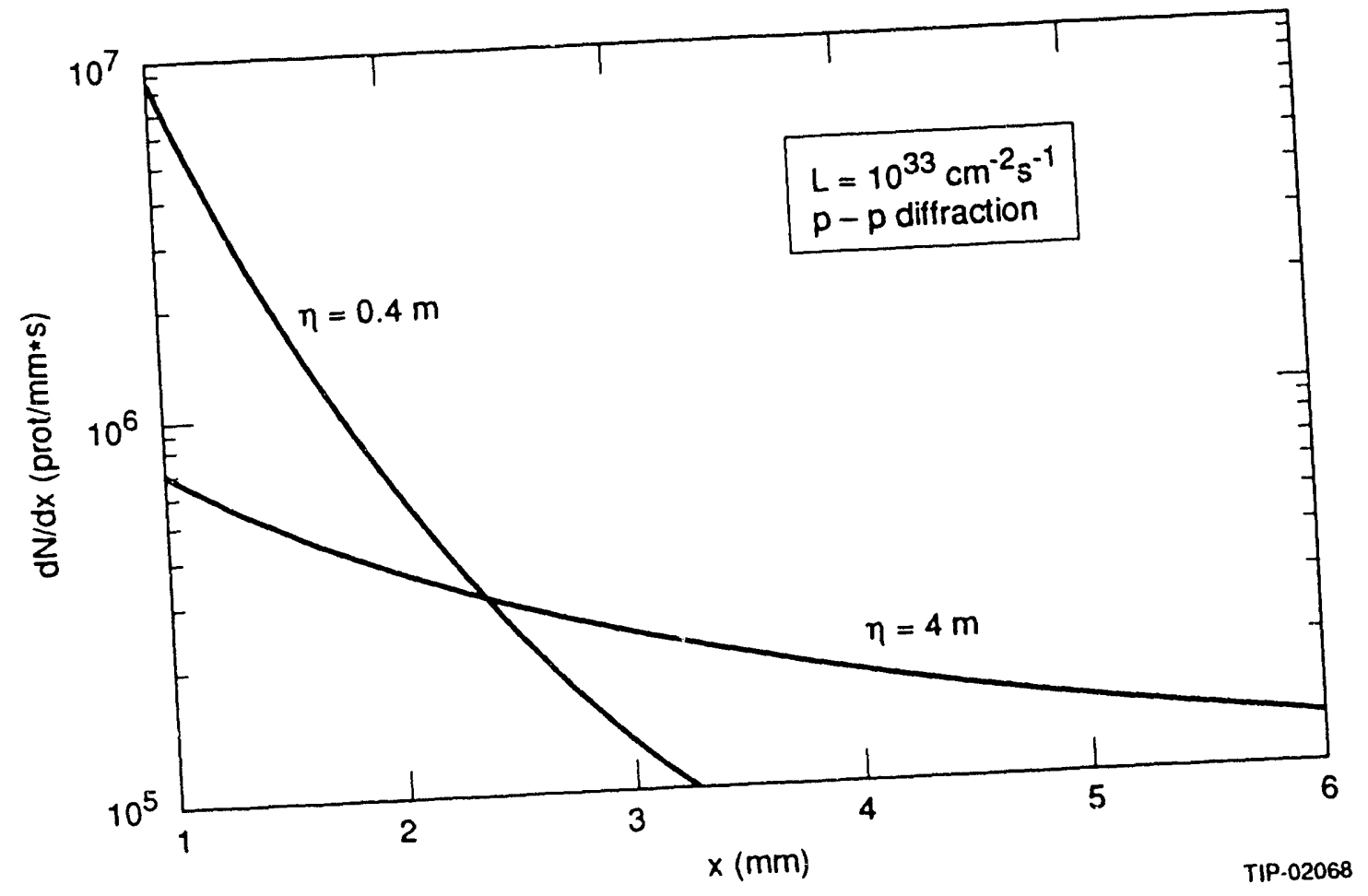

Figure 2. Transverse Distribution of Protons from pp-Diffraction for Two Values of Dispersion. 


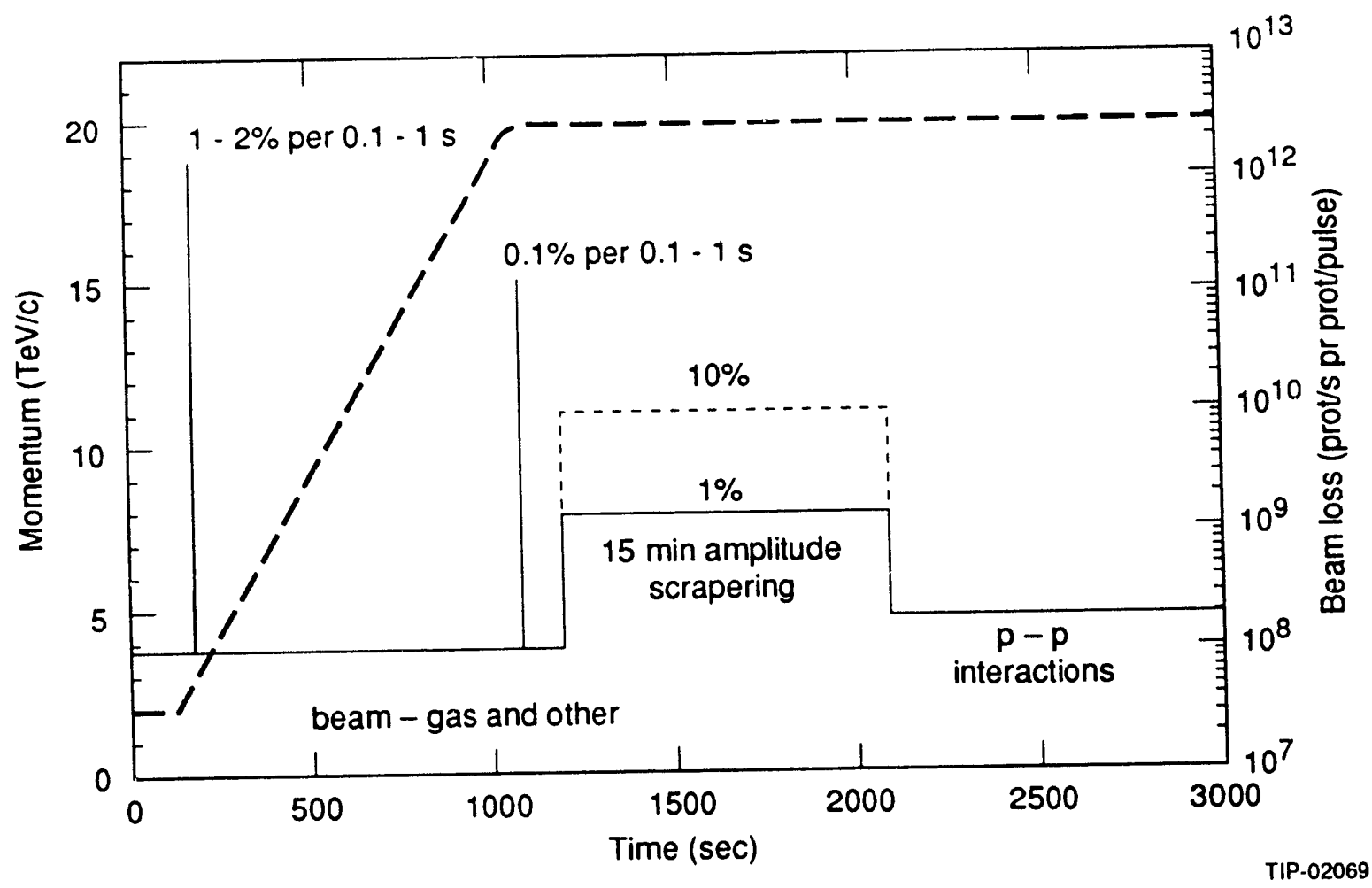

Figure 3. The SSC Beam Loss Scenario.

\subsection{SCRAPING AND MATERIAL CHOICE}

A beam scraping system must minimize beam loss in the machine throughout the cycle, form the required transverse emittance before the collisions, and save this emittance during the whole collider run. Such a system should be capable of intercepting a high halo rate and absorbing most of its energy with minimal effect to the downstream equipment and IP. Most of the above beam halo particles have to be trapped with a scraper at a distance from the beam axis $x_{O}$, which defines the minimum machine aperture (Figure 4). To intercept protons with the large amplitude one needs to put the scraper in the region with the largest $\beta$-function. To trap off-momentum protons a nonzero dispersion at that position is required to provide $\Delta=\eta \Delta p / p \neq 0$ (Figure 4). The primary $x_{0}$ is chosen to be $10 \sigma$ (see Tables 2-5 and Reference 6). To provide favorable experimental conditions, according to Fermilab ${ }^{5}$ and $C E R N^{6}$ experience, this distance should be as small as $6 \sigma$ or even less $(3 \sigma)$. This can be achieved by moving the beam slowly with some velocity in the 3$10 \sigma$ range with the simple bump-magnet (see Section 9.0).

The average impact parameter $\Delta$ of the halo particles at the scraper face grows linearly with this velocity and with the proton cransverse drift speed $V$. Figure 5 shows this dependence for the average and mäimum $\Delta$ for $\beta=400 \mathrm{~m}$ and scraper in final position $=3 \sigma$. According to CERN experience ${ }^{6} V \sim 1 \sigma / \mathrm{s}$ and $6 \sigma / \mathrm{s}$ at $x_{O}=3 \sigma$ and $6 \sigma$, respectively. It corresponds to $V=0.14$ and 
$0.84 \mathrm{~mm} / \mathrm{s}$ for $\beta=400 \mathrm{~m}$, and 0.24 and $1.44 \mathrm{~mm} / \mathrm{s}$ for $\beta=1200 \mathrm{~m}$ (see Table 2). For such velocities the impact parameter $\Delta$ of the halo particles on the scraper is extremely small $(\sim 1-2 \mu \mathrm{m})$, resulting in many difficulties in designing a scraper system.

A scraper cannot intercept all the incident protons because there is a high exit probability for particles traveling close to the material surface that undergo multiple Coulomb scattering and elastic and diffractive scattering. ${ }^{2}$ Having a very small impact parameter presents another problem: an extremely high energy deposition density at the shower maximum in the scraper. So, the following issues must be considered in the design of a scraper system:

- Material integrity: cracking/melting, cooling system, and radiation damage

- Outscattered protons: quenches in downstream magnets

- Outscattered protons: beam size and background in IPs

- Alignment requirements

- Muon vector downstream from the scraper

- Residual radioactivity

- Radiation shielding.

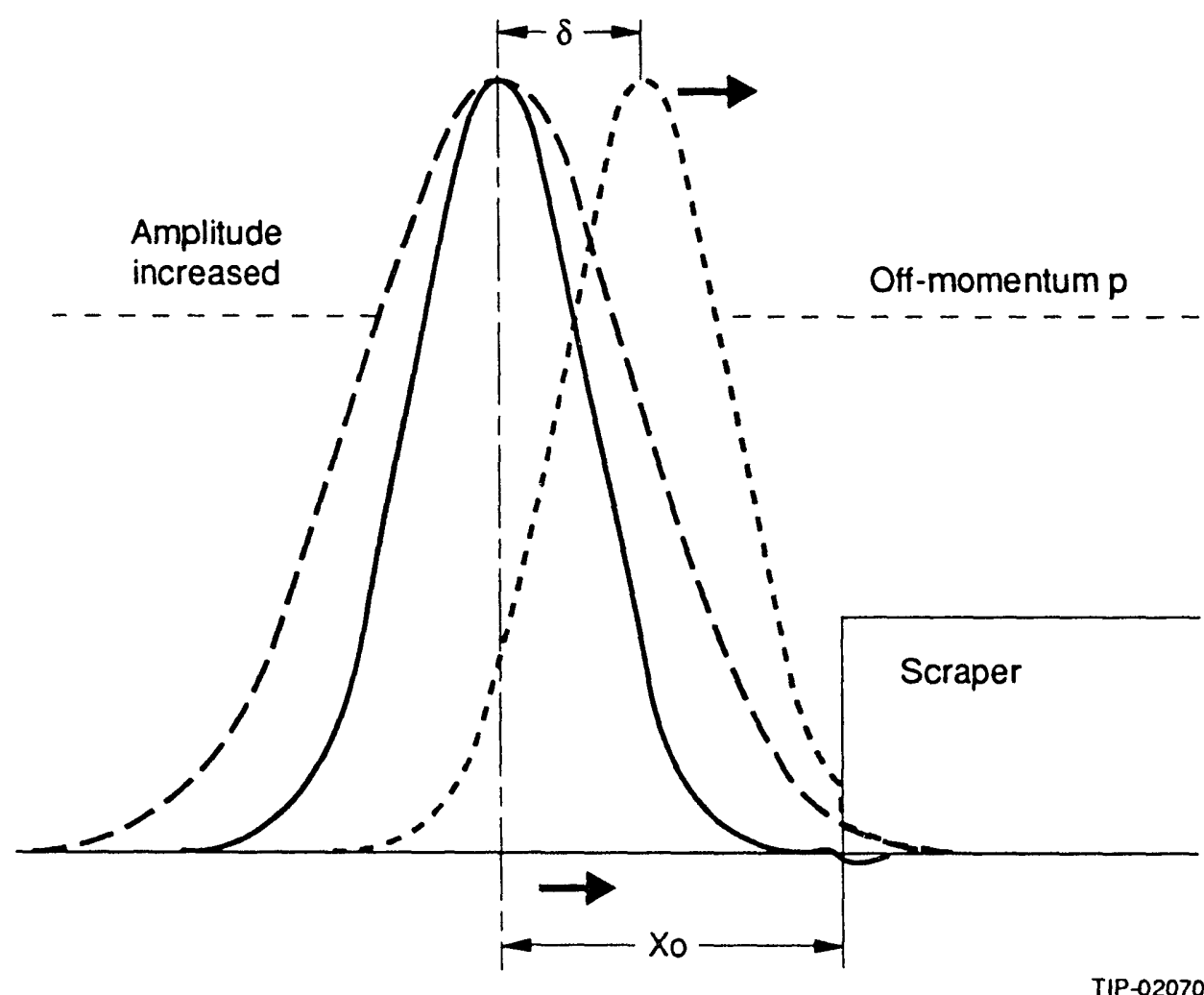

Figure 4. Dynamics of Beam Halo-Scraper Interaction: 1) Beam Lead Up to the Scraper (Solid); 2) Large Amplitude Protons (Long Dash); 3) Off-Momentum Protons (Short Dash). 


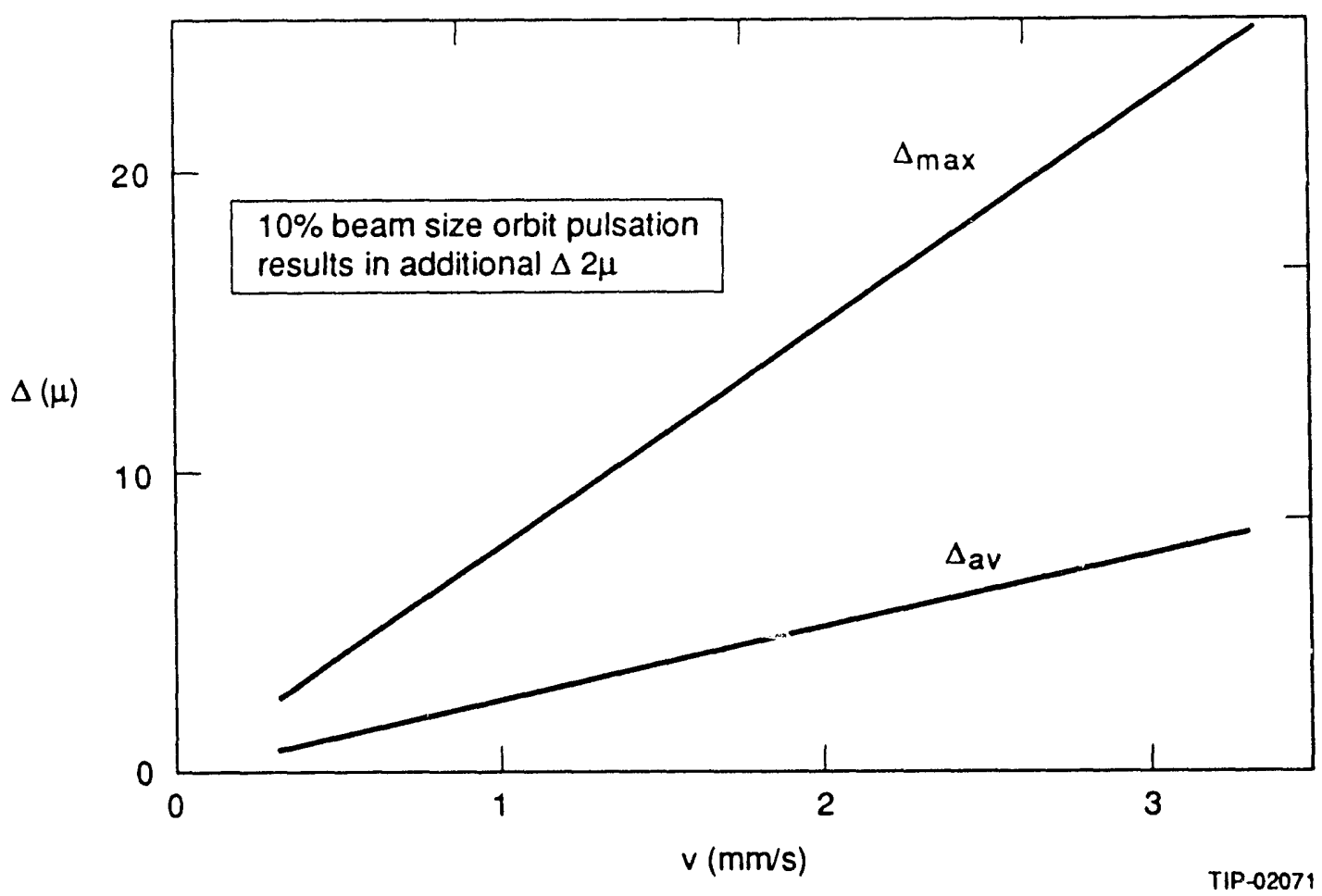

Figure 5. Proton Impact Parameter at the Scraper Versus Transverse Drift Speed.

All the above problems are connected to primary hadronic and electromagnetic cascades in the scraper material. Figure 6 shows the longitudinal distributions of a maximum energy deposition density in tungsten, copper, and graphite scrapers positioned at $\beta=400 \mathrm{~m}$ and $x_{O}=3 \sigma$ for the $20-\mathrm{TeV}$ beam. The transverse drift speed of the halo particles is supposed to be $V=0.67 \mathrm{~mm} / \mathrm{s}$. One can easily see that the maximum deposited energy exceeds the shock wave limits (Table 6) at a rather small number of incident protons. The energy absorbed in the whole scraper is only $20 \%$, even for a large thickness (Figure 7); the rest leaks out of the system and irradiates the downstream elements.

Table 6. Energy Deposition $Q$ and Temperature $T_{\max }$ Limits.

\begin{tabular}{lll}
\hline \hline Material & $\mathrm{Q}(\mathrm{J} / \mathrm{g})$ & $T_{\max }\left({ }^{\circ} \mathrm{C}\right)$ \\
\hline Tungsten & 200 & $\sim 1250$ \\
Copper & 300 & $\sim 740$ \\
Graphite & 1300 & $\sim 1100$ \\
\hline \hline
\end{tabular}

The choice of the scraper material is determined by its ability to absorb a high rate of energy deposition in a limited volume and thus to maintain its integrity. The critical parameters to be 
considered include thermal conductivity properties, lowest yield of the outscattered protons, and an ability to be polished and to remain optically flat. Investigations show that the best candidates are copper and tungsten. Outscattered protons with $\Delta p / p>0.01$ and emittance $>0.11 \pi \mathrm{mm}-\mathrm{mrad}$ (see Tables 1-5) are assumed to be lost in the machine. Figures 8 and 9 present the calculated beam loss of particles with $0.01<\Delta p / p<0.3$ in the whole SSC lattice as a function of the transverse drift speed $V$ and the impact parameter $\Delta$ at the scraper front face. A higher $V$ (or the lead-up velocity) means a larger impact parameter and higher overall scraper efficiency. If the scraper longitudinal thickness is the same in nuclear interaction lengths, there is no significant difference between tungsten $(L=80 \mathrm{~cm})$ and copper $(\mathrm{L}=123 \mathrm{~cm})$ in the meaningful region of velocities and impact parameters.

There is a strong dependence of a proton leakage out of the scraper and correspondingly the beam loss in the machine on the impact parameter in the range 1-100 $\mu \mathrm{m}$ (Figure 9): the beam loss is reduced 10-100 times in this interval. At the same time the beam loss for a copper scraper is about twice that for tungsten at $\Delta>10 \mu \mathrm{m}$. The beam loss in the machine for 2-TeV protons at equal $\Delta$ is about 10 times that for the top energy; thus the largest impact parameters are required during injection.

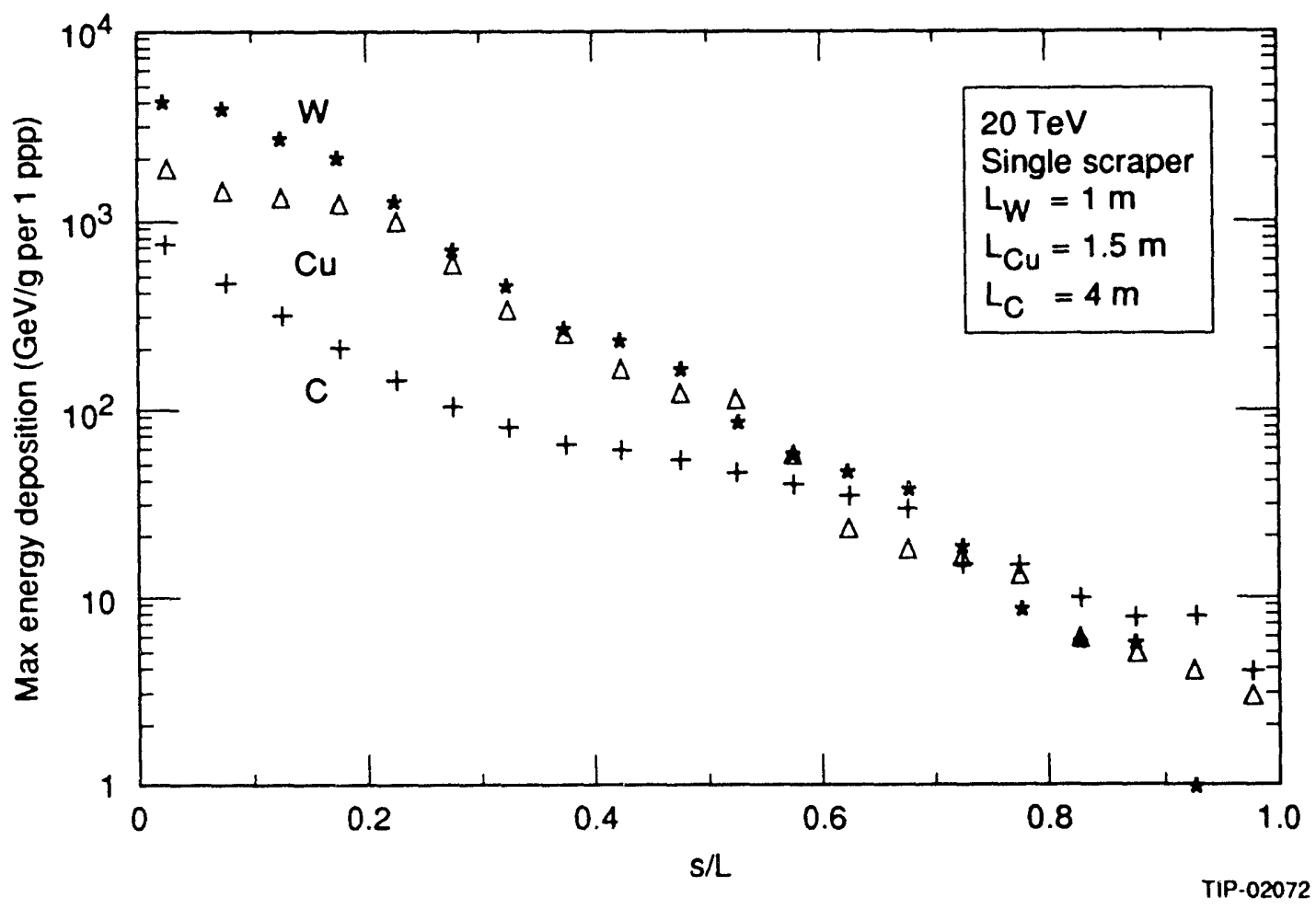

Figure 6. Comparison of the Maximum Energy Deposition Density as a Function of Longitudinal Distance for Tungsten, Copper, and Graphite Scrapers. 


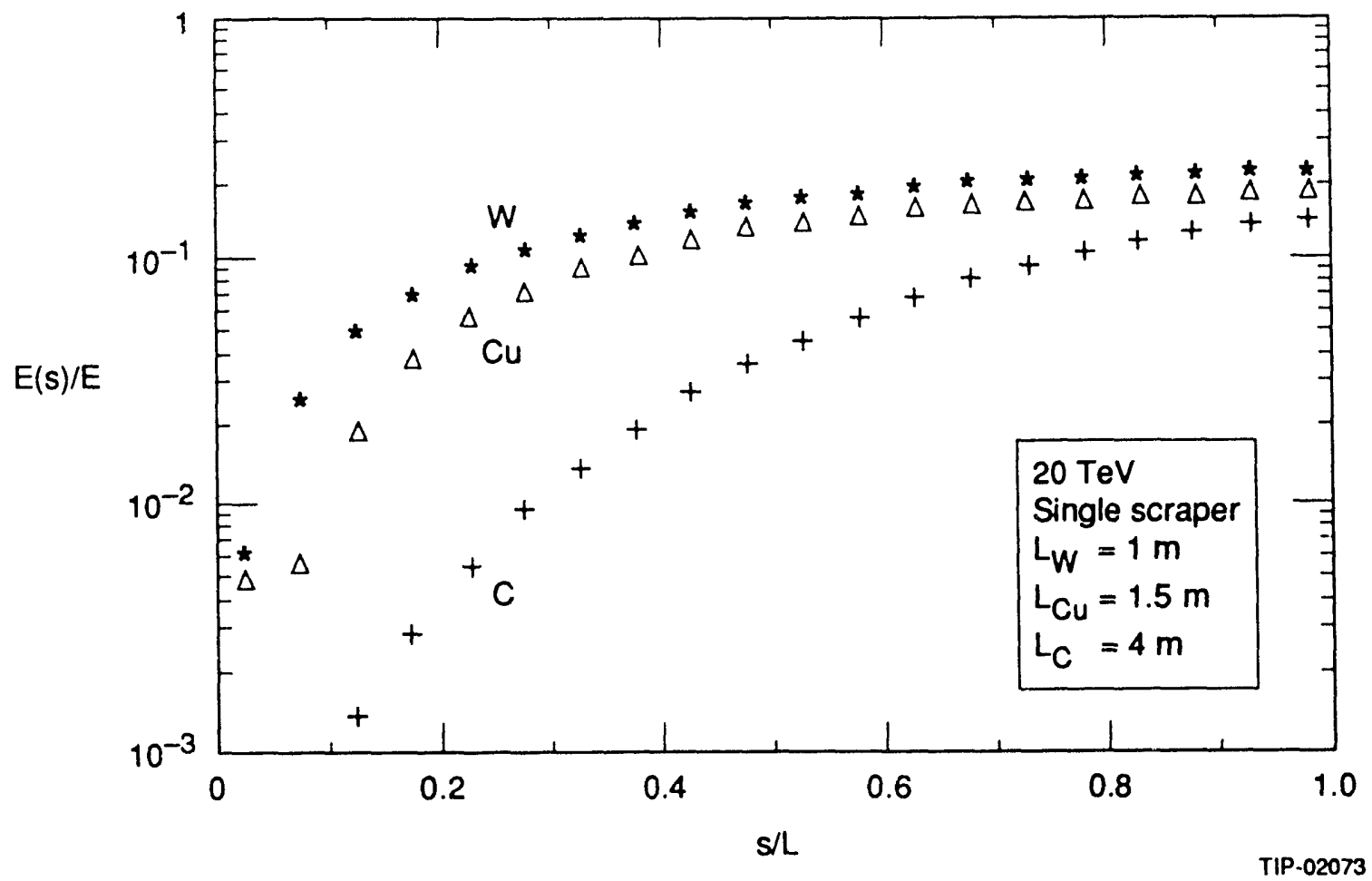

Figure 7. Absorption Efficiency for Scrapers Made of Various Materials as a Function of the Scraper Length.

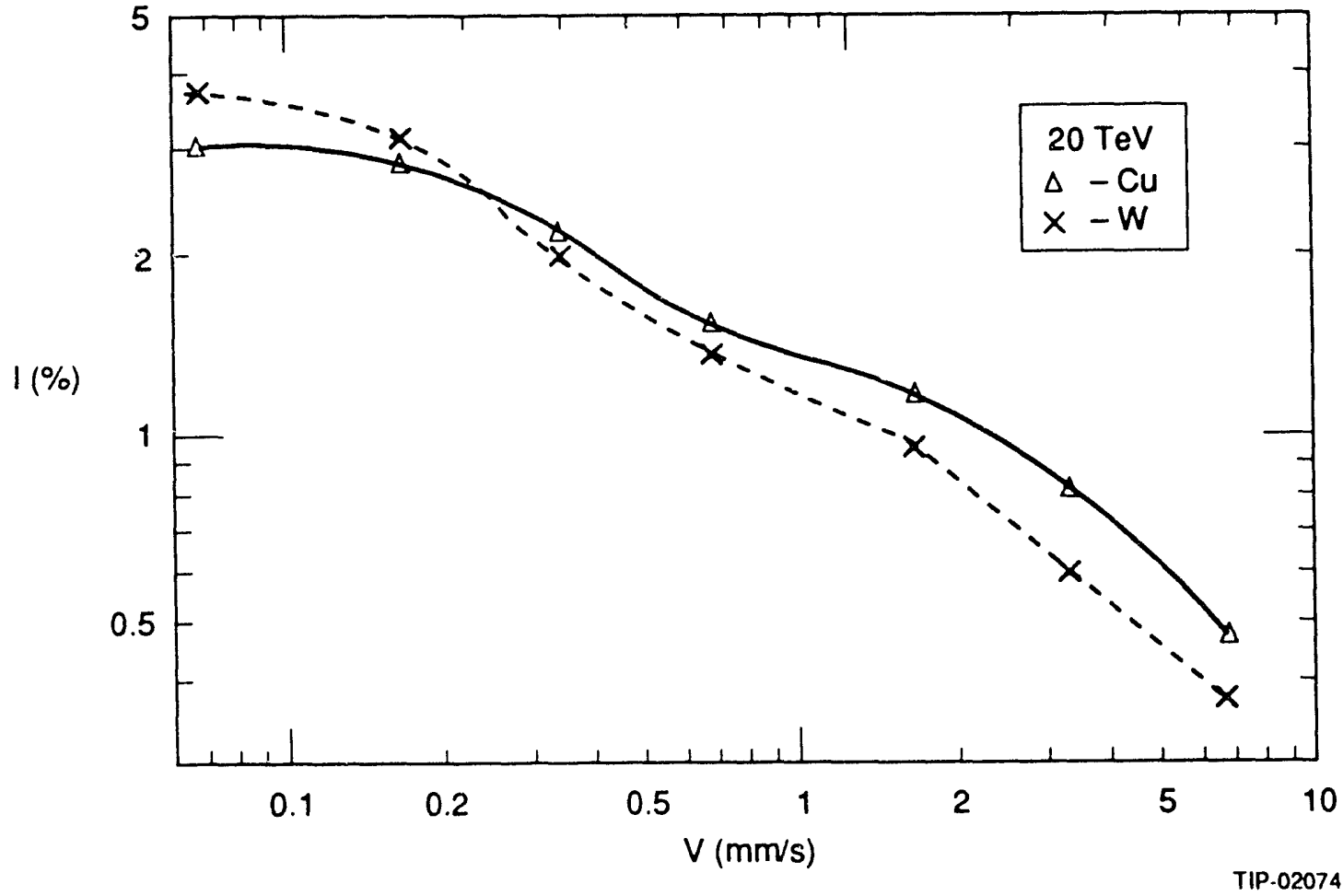

Figure 8. Total Beam Loss in the SSC Lauice Per One Scraped Proton Versus Transverse Drift Speed. 


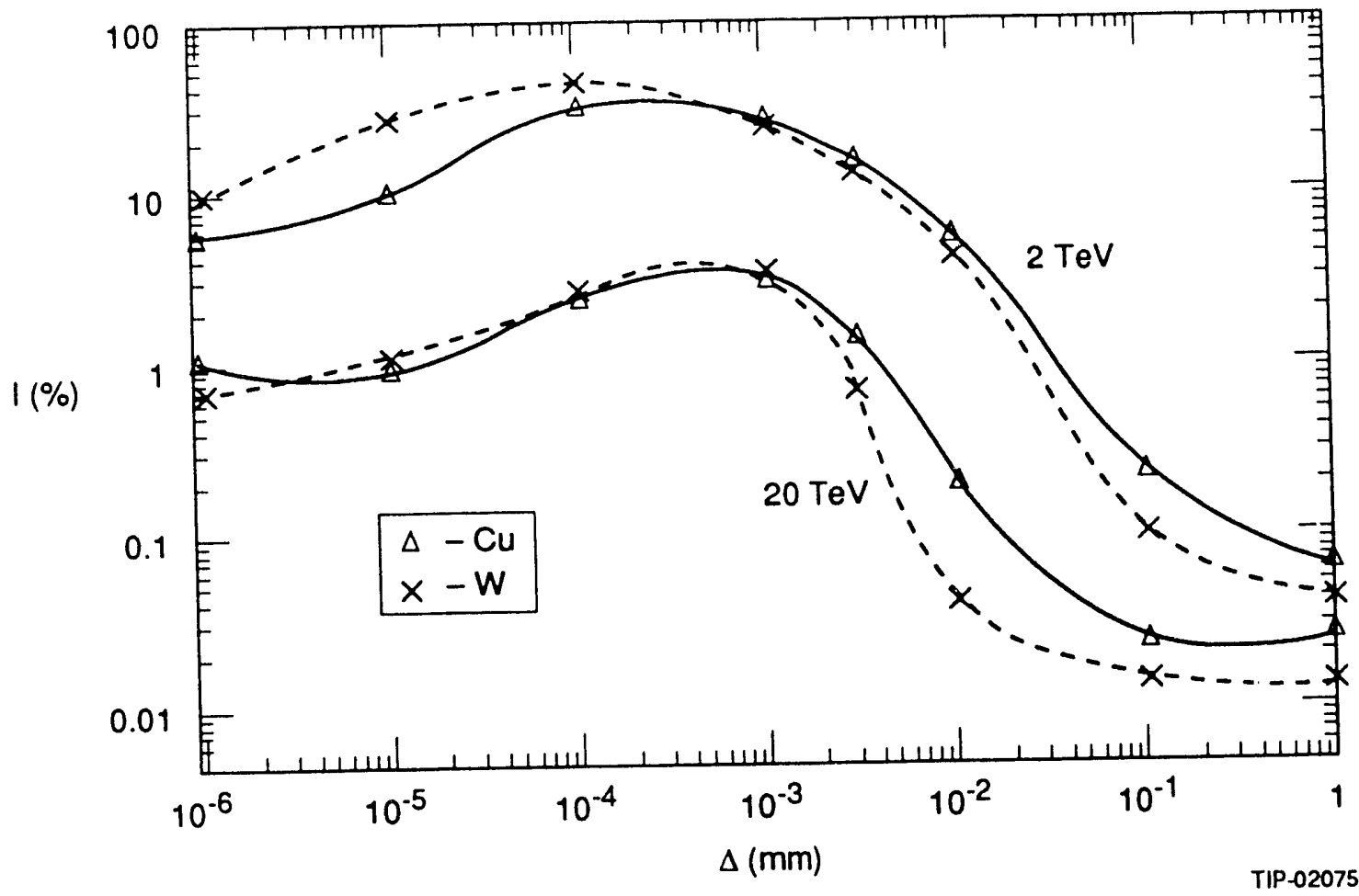

Figure 9. Total Beam Loss in the SSC Lattice Per One Proton Scraped by Tungsten or Copper Scraper at Injection and Full Energy as a Function of the Impact Parameter.

All of the abovt lead to the following conclusions:

- maximum energy deposition density in a tungsten scraper is 2.4 times that in copper, and the maximum allowable scraping rate for a copper scraper is 3.6 times higher compared to tungsten (see Table 8 );

- yield of outscattered protons is almost the same for copper and tungsten scrapers at small impact parameters;

- taking into account the superior heat transfer properties of copper and the cost, one needs to select copper as the best material for scrapers at small impact parameters (with no target, see Section 6.0); it is true also for collimators, though at low intensities of particles at collimators copper can be easily replaced by stainless steel;

- at the same time increasing the transverse displacement of the halo particles at the scraper is very desirable to reduce significantly the beam loss in the machine;

- for such larger impact parameters, the beam loss downstream of the tungsten scraper with a target is about half that of a copper one; so the choice of material for the "primary" scraper should be carefully optimized for the finally selected structure and parameters. 


\subsection{SCATTERING TARGET AND SCRAPER}

High yield of the outscattered protons, leakage of a shower energy, and energy deposition density in the scraper lead to the idea of using a thin target to increase a transverse displacement of the halo particles at the upstream end of the scraper. As a result of multiple Coulomb scattering in the target, protons obtain an increased amplitude and can be intercepted by the scraper on one of the next turns. The target should be rather thin to decrease the probability of nuclear interactions in it and to minimize the energy deposition, but it should be thick enough to provide a large displacement of protons on the scraper in a few turns. First estimation showed significant increase of the impact parameter with the help of a tungsten target of a few millimeters installed at the front face of the scraper and positioned at about half- $\sigma(50-100 \mu \mathrm{m})$ closer to the beam than the inner scraper edge. As demonstrated in Figure 9, use of such a target is outstanding, especially at the injection energy.

Scraping efficiency can also be increased by use of a bent crystal instead of a scattering target. Some results are presented below for such a case, but we feel a few other aspects should be clarified before seriously considering this possibility for the scraper application.

A possible solution for the device is shown in Figure 10. The cylindrical scraper (about $1 \mathrm{~m}$ long and $7 \mathrm{~cm}$ in diameter with a $0.5-1 \mu \mathrm{mm}$ tungsten disc of $100 \mu \mathrm{m}$ larger diameter at the front) is surrounded with a steel shield (see below). Calculated distributions of the impact parameter for 20-TeV protons are presented in Figure 11 for the cases with no target and with a tungsten target of two thicknesses. The difference in the $\Delta$ distribution is drastic: $\langle\Delta\rangle=2 \mu \mathrm{m}$ in the classical case and $\langle\Delta\rangle=100-280 \mu \mathrm{m}$ when one uses a target. It was shown in the previous paper ${ }^{3}$ that the optimum length of the scraper for the SSC parameters is $\sim 80 \mathrm{~cm}$ for tungsten and $\sim 120 \mathrm{~cm}$ for copper. To study system efficiency, a few cases are considered below for the scraper located at $\beta=400 \mathrm{~m}$ both for injection and top energies:

Case 1. No target; copper (1.2-2 m), tungsten $(0.8-1 \mathrm{~m})$ and graphite $(4 \mathrm{~m})$ scrapers.

Case 2. 1-mm tungsten target at the front of copper scraper.

Case 3. 5-mm silicon bent crystal at the front of copper scraper.

Figures 12 and 13 show the longitudinal distribution of maximum energy deposition in the copper scraper for all three cases (see also Figures 6 and 7). Cumulative distributions of the energy absorbed in 7-cm-diameter copper scraper and in the first $5 \mathrm{~cm}$ of the surrounding steel are presented in Figures 14 and 15. One can see a factor of 2.5 reduction in energy density when introducing the target for both energies and 4.3 when using the bent crystal. At the same time the total intercepted energy is increased and is practically independent of the length above $1.2 \mathrm{~m}$ of copper. 


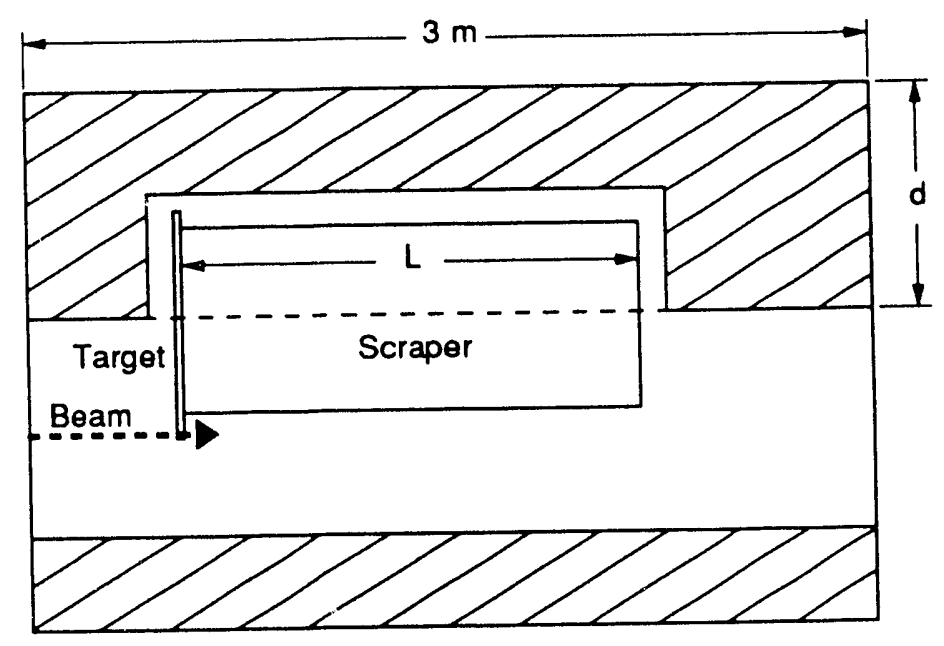

(a)

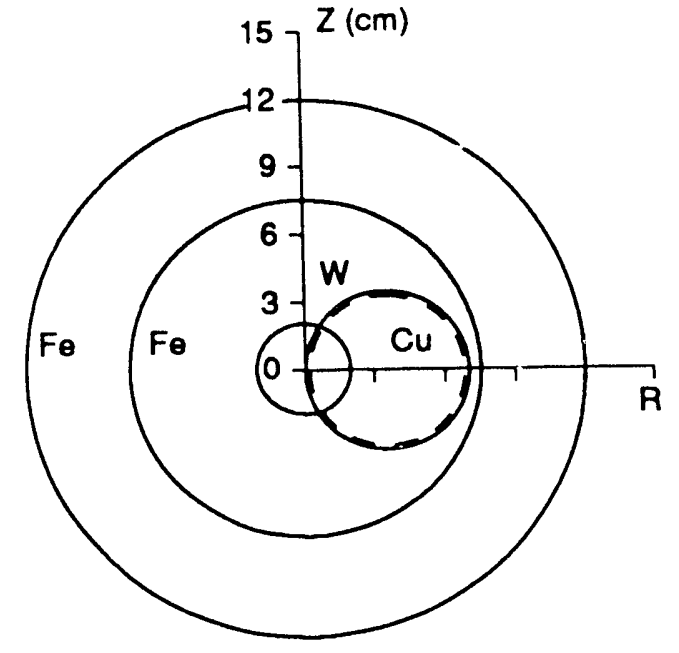

(b)

TIP-02076

Figure 10. Schematic of Scraper, Target and Radiation Shielding: a) Longitudinal View; b) Transverse Cross Section.

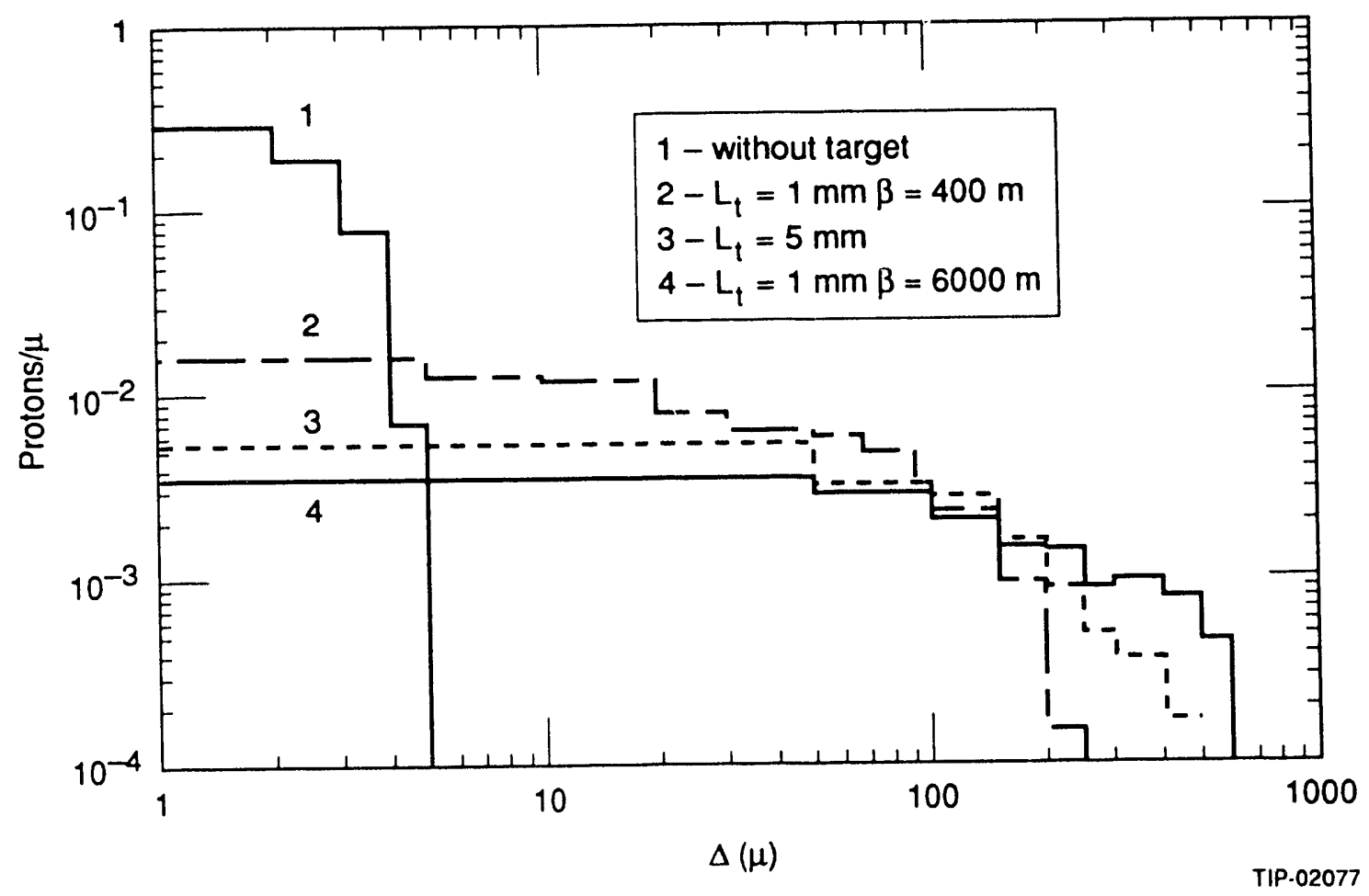

Figure 11. Comparison of Proton Impact Parameter Distribution at the Scraper Upstream End for the Different Scraping Schemes With and Without a Scattering Target. Note effectiveness of target in spreading impact distribution. 


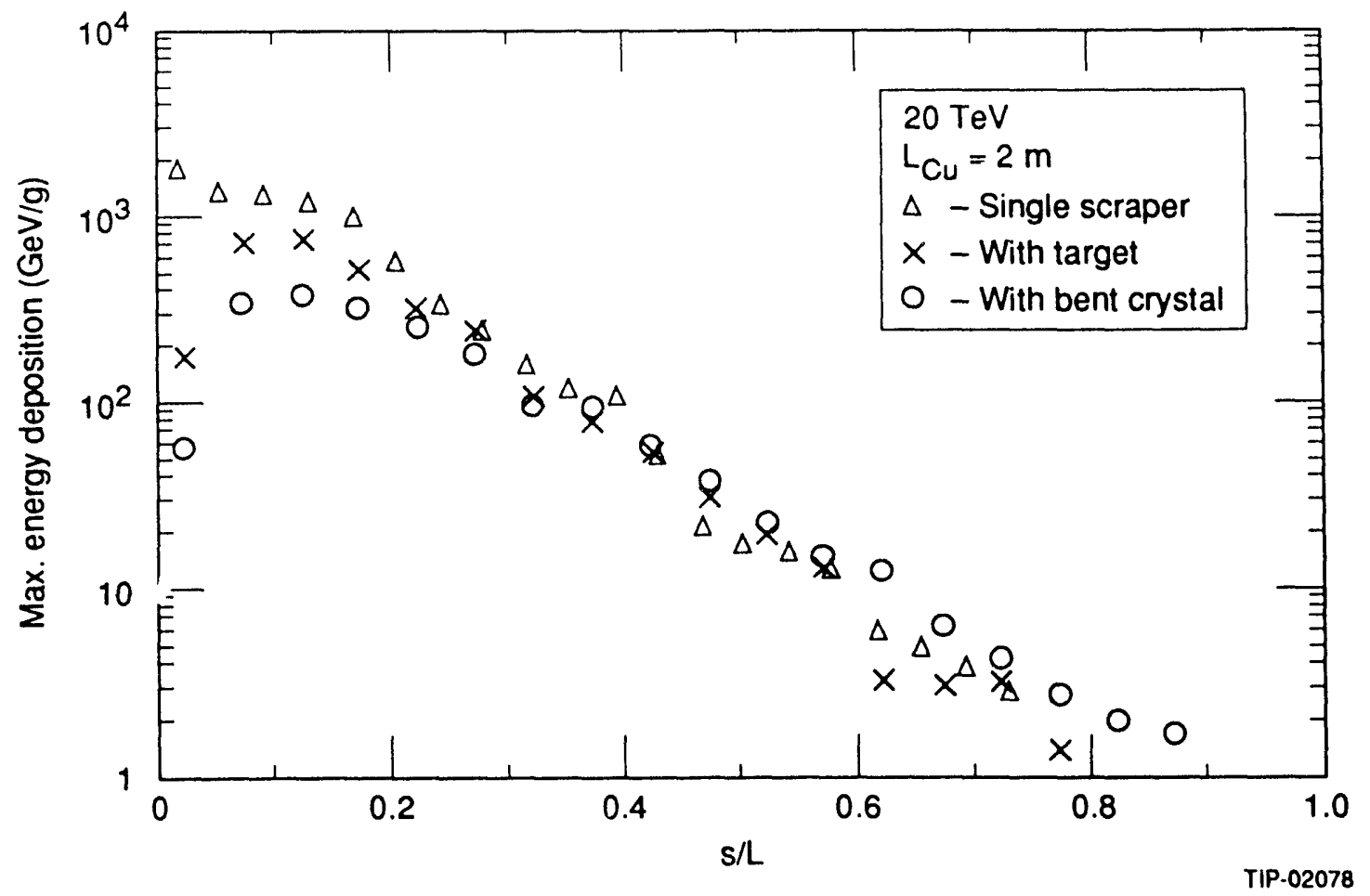

Figure 12. Maximum Energy Deposition Per Scraped Proton $(20 \mathrm{TeV})$ as a Function of Longitudinal Distance in Copper Scraper: With No Target, With 1-mm Tungsten Target, and With 5-mm Silicon Bent Crystal at the Front of the Scraper. Note dramatic decrease of energy deposition for first bin.

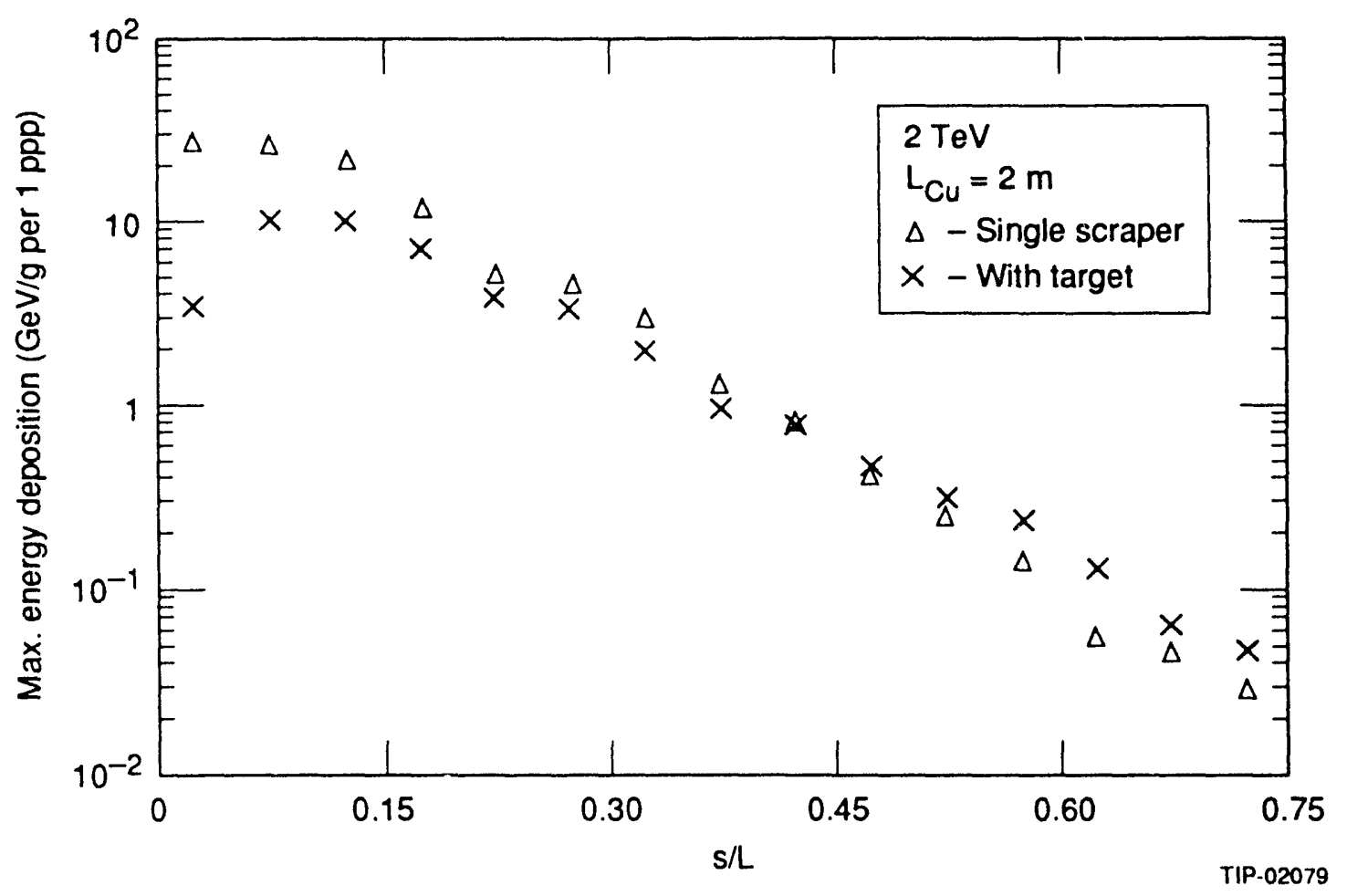

Figure 13. Maximum Energy Deposition Per Scraped Proton (2 TeV) as a Function of Longitudinal Distance in Copper Scraper: With No Target and With 1-mm Tungsten Target. 


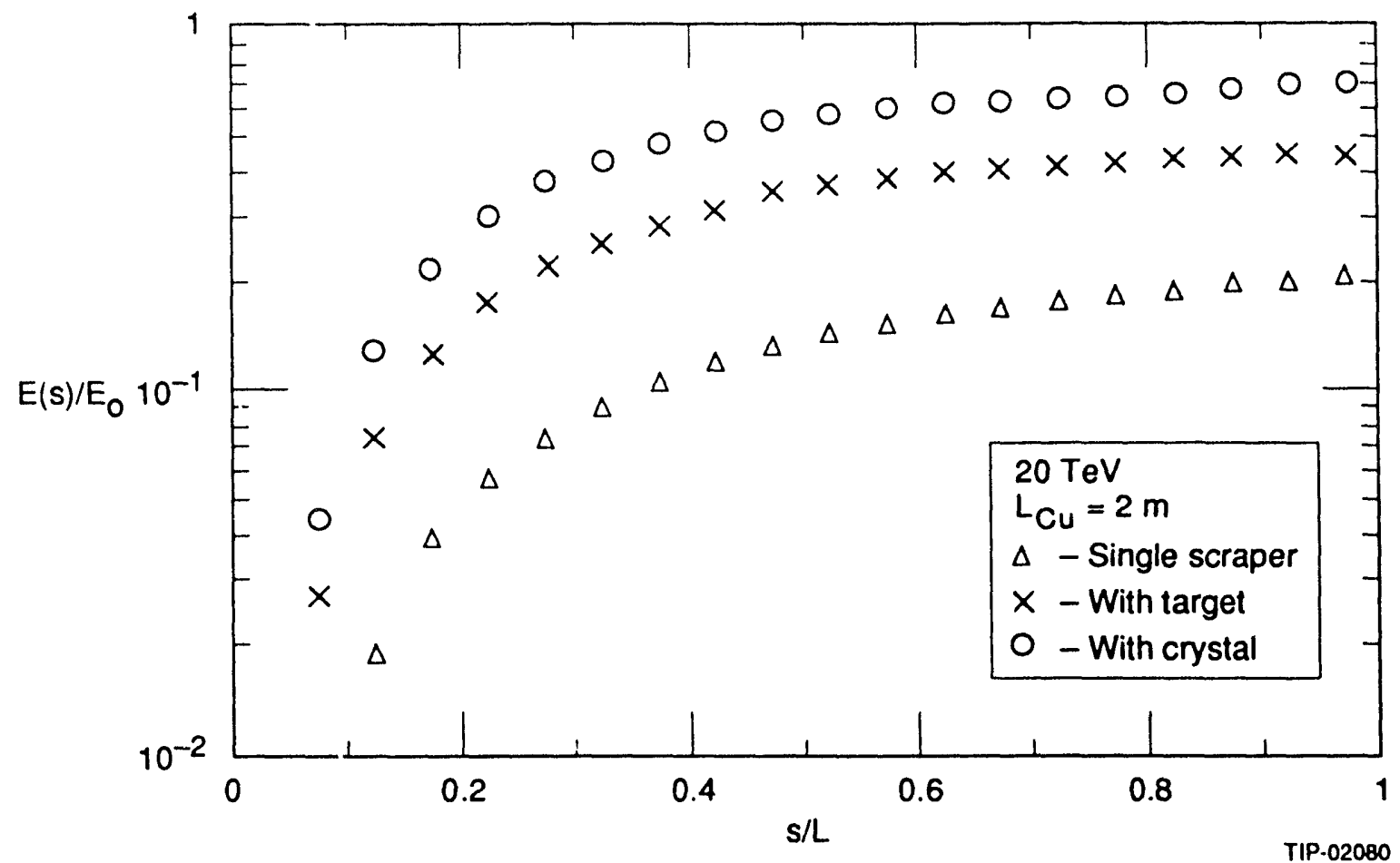

Figure 14. Absorption Efficiency of Copper Scraper as a Function of Scraper Length for Different Scraping Modes at $20 \mathrm{TeV}$.

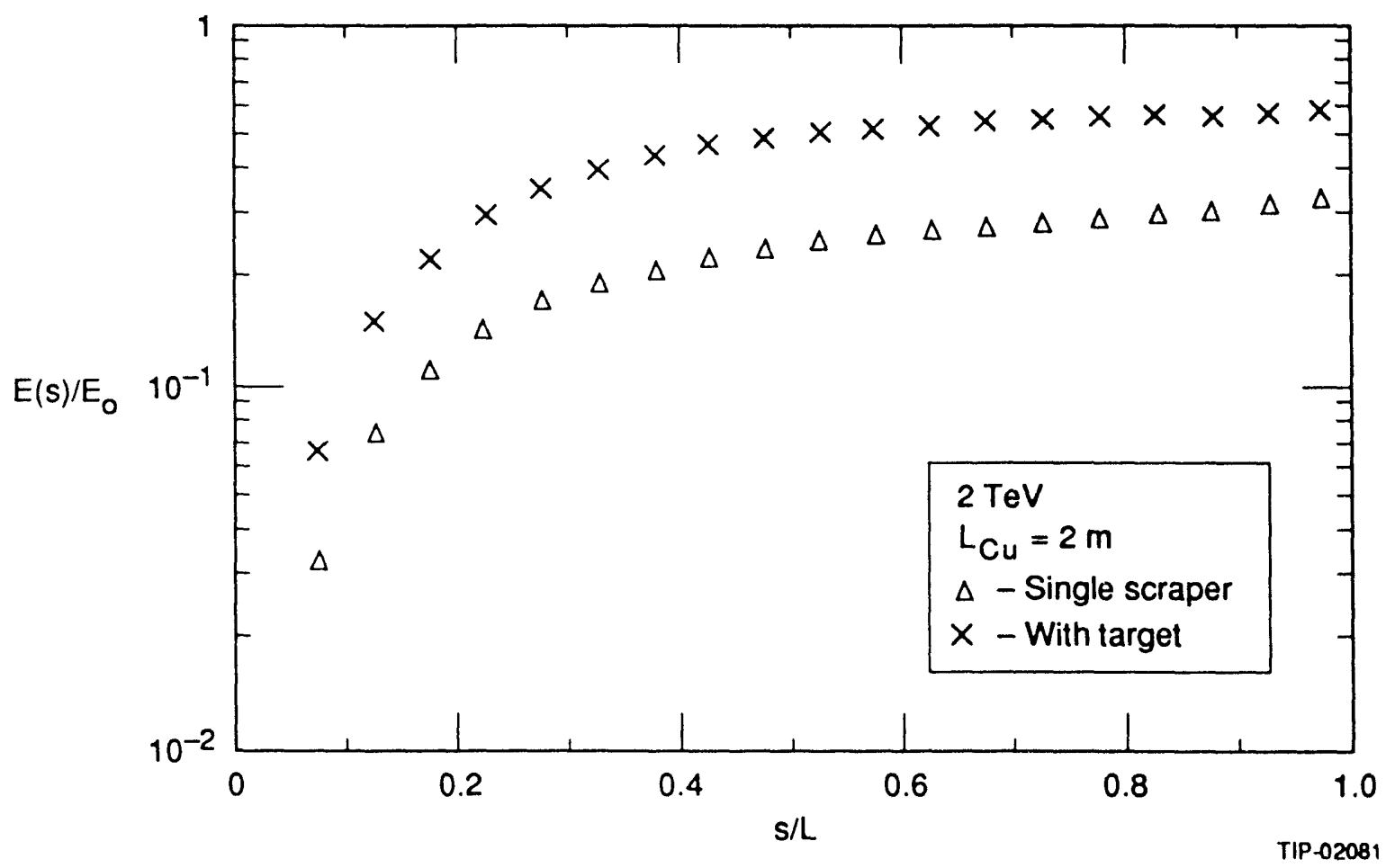

Figure 15. Absorption Efficiency of Copper Scraper as a Function of Scraper Length for Different Scraping Modes at $2 \mathrm{TeV}$. 
For all the cases considered above, the contributions to the proton yield from elastic scattering and other processes with various momentum transfer are presented in Table 7 . Notice the similarity of elastically scattered proton yields for tungsten and copper scrapers and the rather large difference in the other cases. The yield is lower by a factor of 20 when one puts a target at the front face of the scraper. The leakage angle for protons, which are candidates for the longrange loss $(\Delta p / p<0.01)$ doesn't exceed $50 \mu \mathrm{rad}$ at $20 \mathrm{TeV}$ and $1 \mathrm{mrad}$ at the injection energy.

Table 7. Number of Outscattered Protons (\%) per 1 Incident Proton and Yield Angle.

\begin{tabular}{lllllll}
\hline$E(T e V)$ & Case & $\begin{array}{l}\text { Scraper } \\
\text { material }\end{array}$ & Elastics & $\begin{array}{l}\text { Others } \\
<0.001\end{array}$ & $\begin{array}{l}\text { with } \\
<0.01\end{array}$ & $\begin{array}{l}\Delta \mathrm{p} / \mathrm{p} \\
<0.3\end{array}$ \\
\hline 20 & 1 & $\mathrm{Cu}$ & 4.460 & 0.026 & 0.053 & 2.520 \\
& 1 & $\mathrm{~W}$ & 4.160 & 0.150 & 0.347 & 1.730 \\
& 1 & $\mathrm{C}$ & 8.640 & 0.915 & 1.710 & 6.680 \\
& 2 & $\mathrm{Cu}$ & 0.240 & 0.020 & 0.031 & 0.175 \\
& 3 & $\mathrm{Cu}$ & 0.005 & 0 & 0 & 0.007 \\
\hline 2 & 1 & $\mathrm{Cu}$ & 6.920 & 0.154 & 0.551 & 3.140 \\
& 2 & $\mathrm{Cu}$ & 0.170 & 0.003 & 0.007 & 0.154 \\
\hline Yield angle & $(\mathrm{mrad})$ & at 20 TeV & $<0.05$ & $<0.05$ & $<0.05$ & $<0.3$ \\
& & at 2 TeV & $<1$ & $<1$ & $<1$ & $<3$ \\
\hline \hline
\end{tabular}

It was found that the best value for the target-scraper off-set is $50 \mu \mathrm{m}$, and the optimal tungsten target thickness is $0.5 \mathrm{~mm}$ for $\beta$-function $\sim 1200 \mathrm{~m}$ at the target/scraper position (see Section 8.0 ). About $1.5 \%$ of the protons intercepted by the scraper interact inelastically with the target nuclei. For the smaller $\beta$-function the optimal target thickness scales crudely with $1 / \beta$. The amplitude and momentum distributions of protons to be lost in the machine (A>20 mm in the arcs) are presented in Figures 16 and 17 for an $80-\mathrm{cm}$ tungsten scraper at $\beta=1200 \mathrm{~m}$ with and without a $0.5-\mathrm{mm}$ tungsten target. By introducing the target one decreases the total beam loss by about a factor of 10 .

Distributions of the transverse displacement of protons on the target and $80-\mathrm{cm}$ tungsten scraper at $\beta=1200 \mathrm{~m}$ are shown in Figures 18 and 19 for two transverse drift speeds $V$ at the final extreme position of the target $x_{O}=0.8 \mathrm{~mm}$ (corresponds to $3 \sigma$ ). One can see how wide the distribution is on the scraper compared to that on the target. 


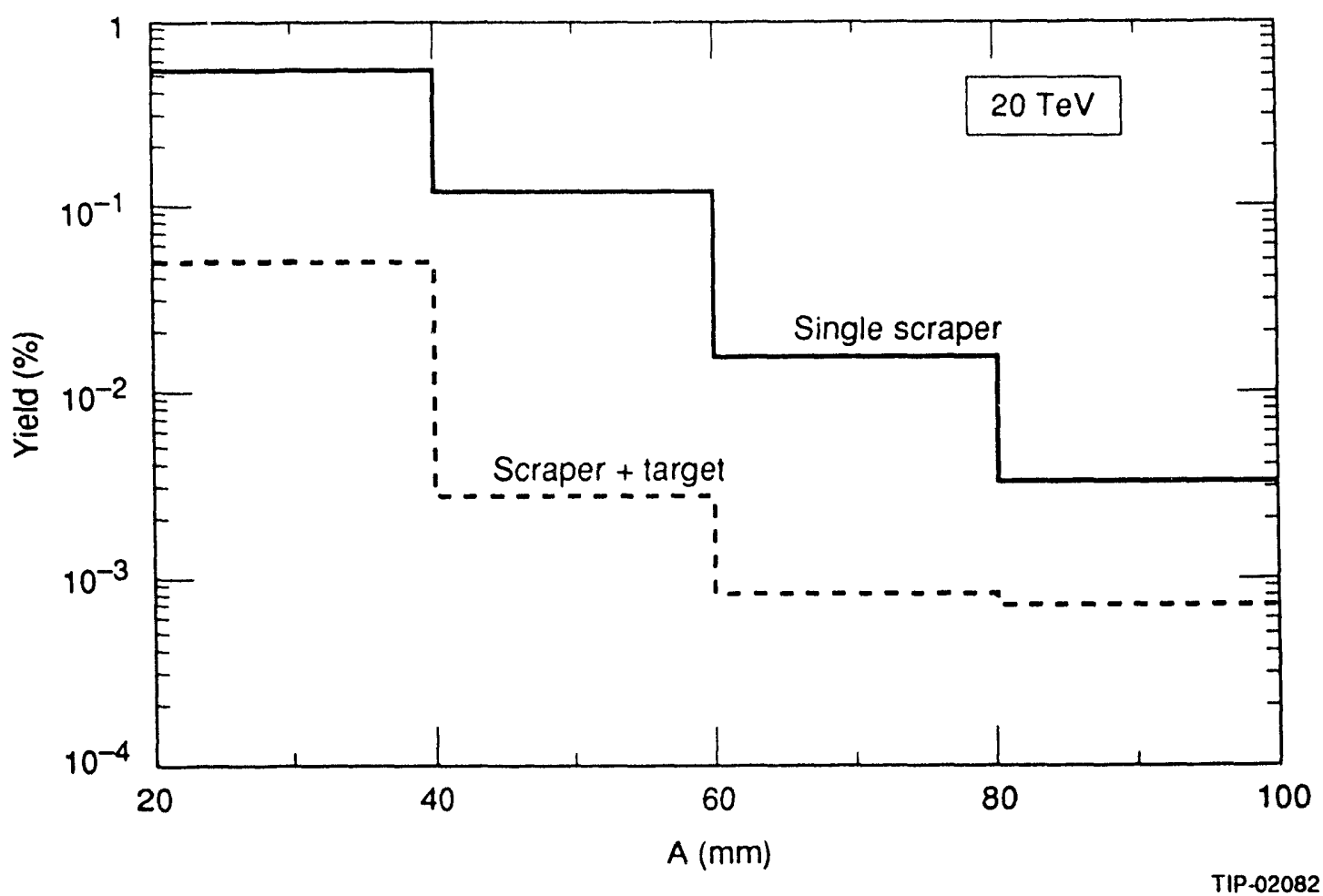

Figure 16. Amplitude Distribution of Outscattered Protons to be Lost on the Machine Aperture for Scraping at 20 TeV With and Without Target.

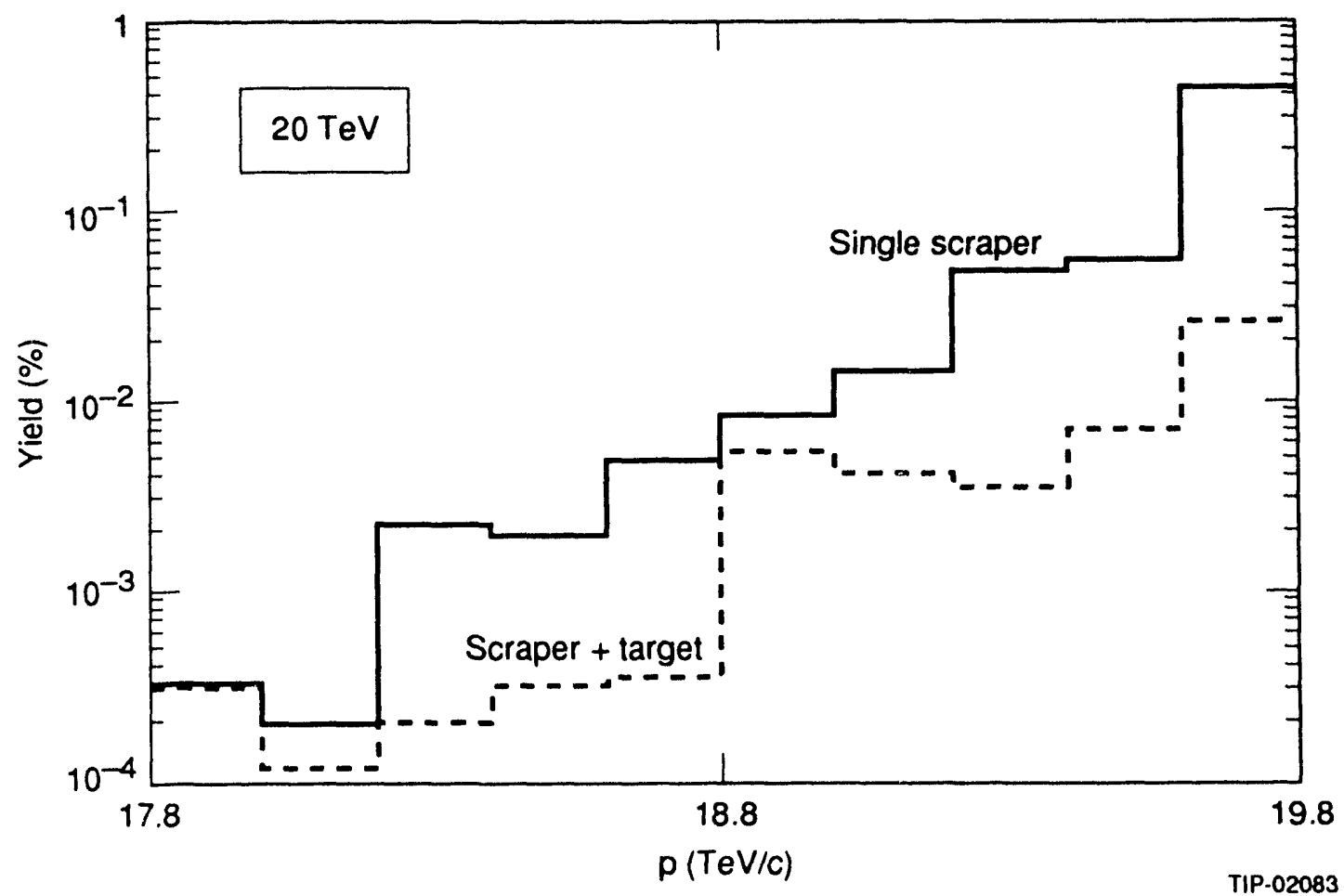

Figure 17. Momentum Distribution of Outscattered Protons for Scraping at $20 \mathrm{TeV}$ With and Without Target. 


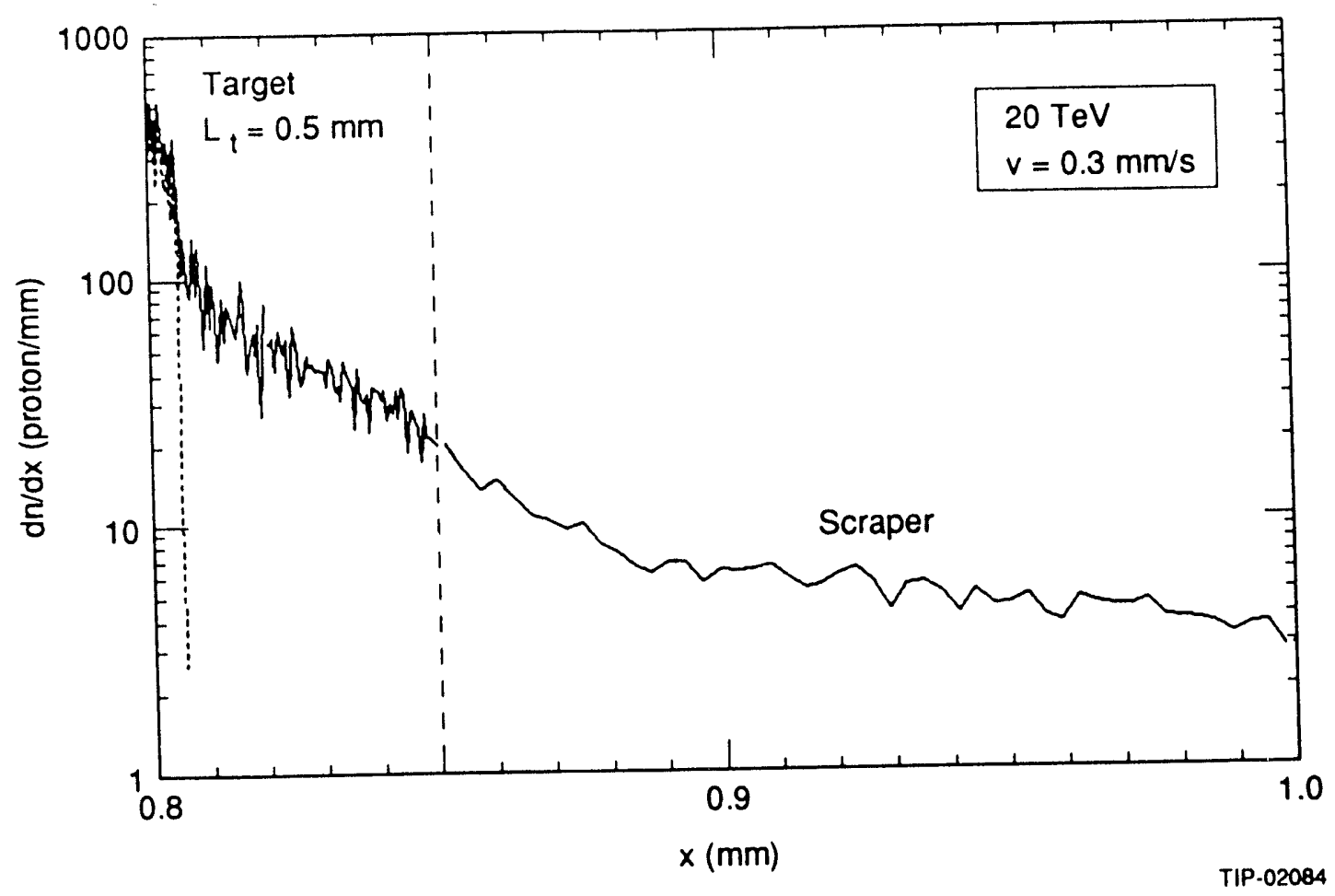

Figure 18. Proton Transverse Displacement Distribution on Target (Short Dash) and Scraper (Solid) at $0.3 \mathrm{~mm} / \mathrm{s}$ Transverse Drift Speed. Target inner edge is positioned at $0.8 \mathrm{~mm}$ and the scraper edge at $0.85 \mathrm{~mm}$.

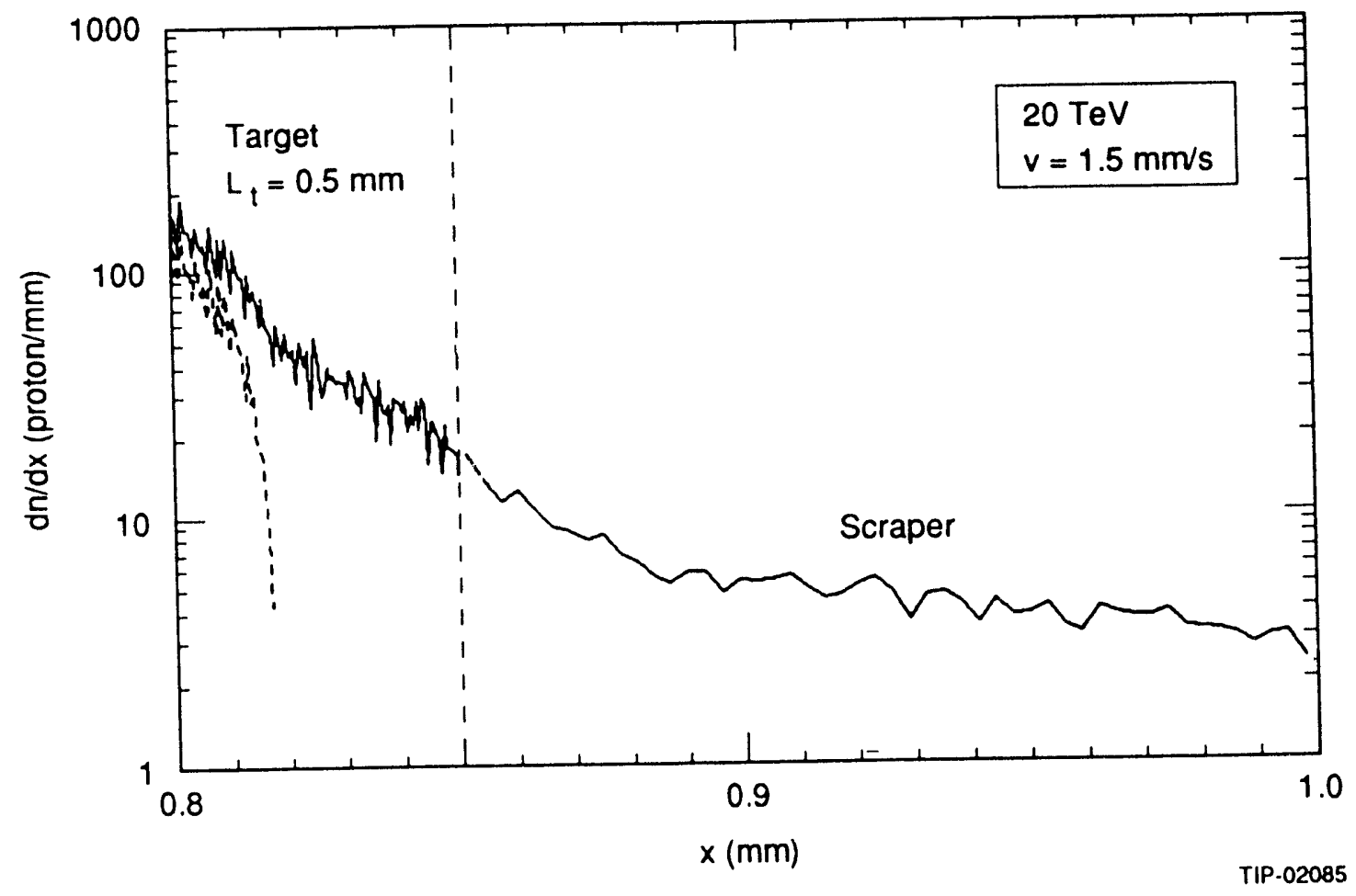

Figure 19. Proton Transverse Displacement Distribution on Target (Short Dash) and Scraper (Solid) at $1.5 \mathrm{~mm} / \mathrm{s}$ Transverse Drift Speed. Target inner edge is positioned at $0.8 \mathrm{~mm}$ and the scraper edge at $0.85 \mathrm{~mm}$. 
The distribution of the number of target crossings by halo protons before scraper interception and the consequent number of turns in the lattice after the first pass through the target are shown in Figure 20. One should notice that the protons do pass through the target an average of 2-3 times before interaction with the scraper, and it takes $20-30$ revolutions in the machine.

Figure 21 shows the total beam loss in the SSC lattice $(\Delta p / p>0.01$ and emittance $>0.11 \pi$ $\mathrm{mm}$-mrad) per one proton intercepted with the $80-\mathrm{cm}$ tungsten scraper as a function of the alignment angle. As one might expect, the beam loss for the scraper with target is less than 10\% of that with no target, and dependence on the angle between the beam axis and the inner edge of the scraper is much flatter.

So, introducing the scattering target in the scraper system results in:

- a factor of 2.5 reduction of maximum energy deposition density that simplifies the thermal conditions in the scraper material and extends its lifetime;

- an increase of total halo beam energy absorbed in the scraper (50\% vs $20 \%$ );

- a factor of 10 decrease of total beam loss downstream of the scraper, and

- a significant relaxation of requirements to the scraper surface finish and alignment.

All of these mean a considerable improvement in the scraper system efficiency.

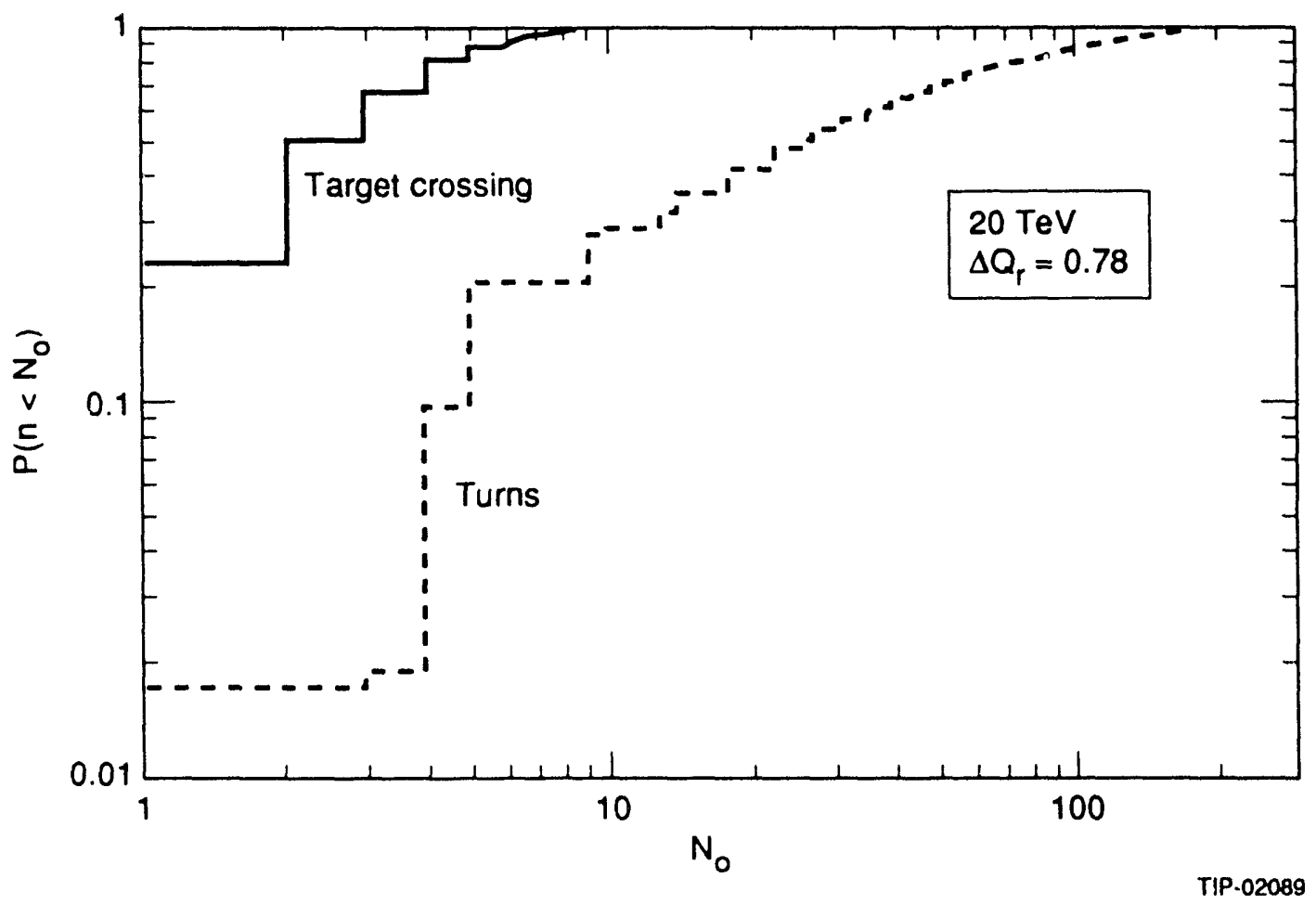

Figure 20. Probability of Target Crossing and Number of Turns After the First Pass Through the Target as a Function of Number of Turns. 


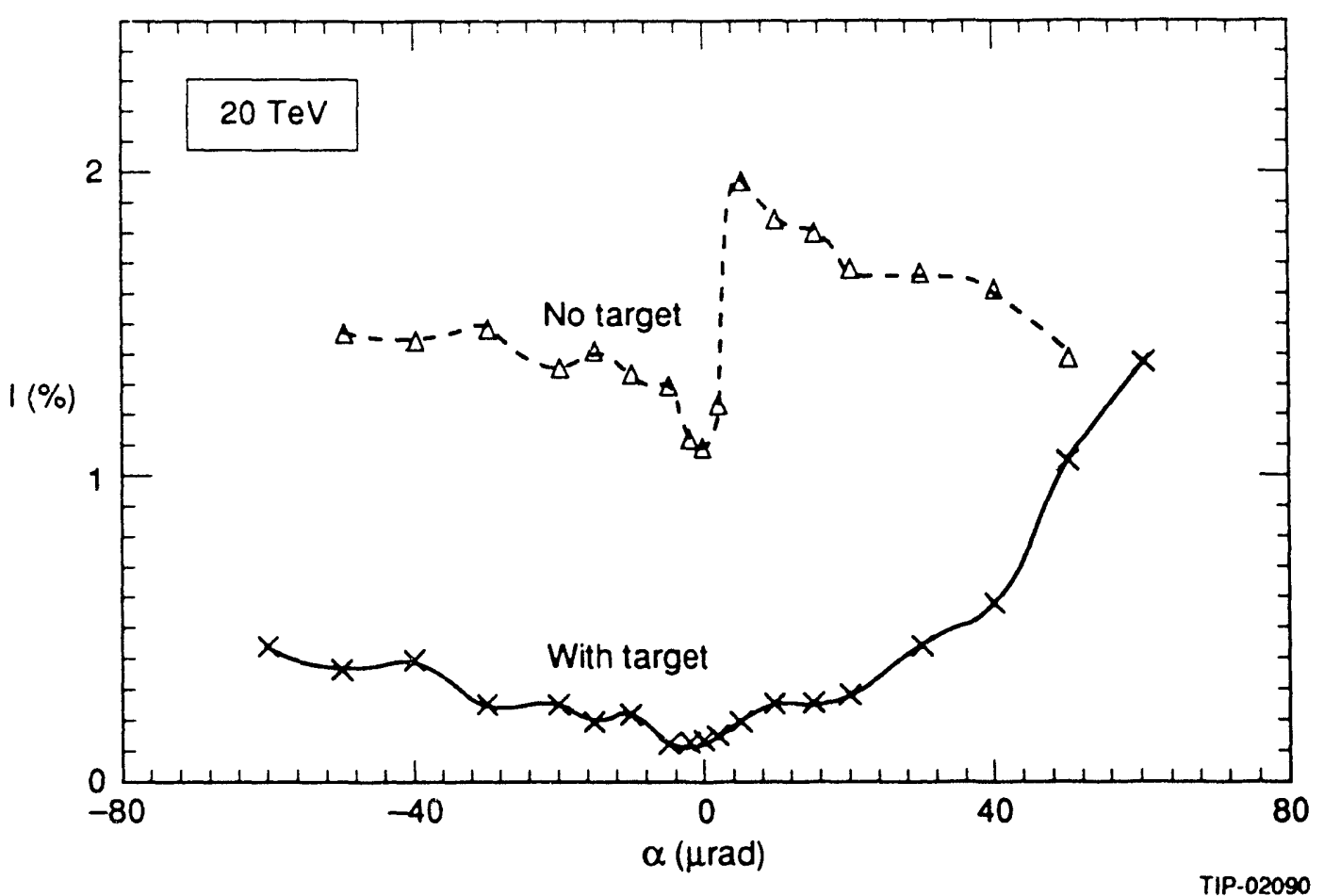

Figure 21. Total Beam Loss in the SSC Lattice Per One Proton Scraped by Tungsten Scraper Versus Scraper Alignment Angle.

\subsection{SCRAPING RATE}

With energy deposition data of the above section as input and taking the numbers of Table 6 as the limits, we obtain the maximum allowable number of protons per instantaneous spill for all cases under consideration (Table 8). The energy deposition density in a thin tungsten target is about $100 \mathrm{GeV} / \mathrm{g}$ per one $20-\mathrm{TeV}$ proton; this results in $10^{10}$ protons per fast spill, which causes destruction of limited region in the target. For a $2-\mathrm{TeV}$ beam this number is a few by $10^{11}$. To increase the ability of the system to accept larger beam intensities, we suggest making a target/scraper couple that is stepwise rotatable (see Figure 10). This spreads the beam over a larger area and helps to extend the system lifetime by simplifying the radiation damage problem. A single spill on the target at $20 \mathrm{TeV}$ results in the maximal dose in copper scraper equal to 30 Mrad. 
Table 8. Maximum Energy Deposition in the Scraper and Number of Protons in the Fast Spill Causing the Material Destruction.

\begin{tabular}{lllll}
\hline \hline$E(T e V)$ & Case & $\begin{array}{l}\text { Scraper } \\
\text { material }\end{array}$ & $\begin{array}{l}E_{\text {dep }} \\
(\mathrm{GeV} / \mathrm{g})\end{array}$ & $N_{\max }(\mathrm{ppp})$ \\
\hline \multirow{2}{*}{20} & 1 & $\mathrm{~W}$ & 4300 & $2.91 \times 10^{8}$ \\
& 1 & $\mathrm{Cu}$ & 1800 & $1.04 \times 10^{9}$ \\
& 1 & $\mathrm{C}$ & 730 & $1.11 \times 10^{10}$ \\
& 2 & $\mathrm{Cu}$ & 750 & $2.50 \times 10^{9}$ \\
& 3 & $\mathrm{Cu}$ & 420 & $4.46 \times 10^{9}$ \\
\hline 2 & 1 & $\mathrm{Cu}$ & 27 & $6.94 \times 10^{10}$ \\
& 2 & $\mathrm{Cu}$ & 10 & $1.88 \times 10^{11}$ \\
\hline
\end{tabular}

One can see that the maximum allowable number of protons per fast spill for the same conditions is in the ratio 1:3.6:38 for tungsten, copper and graphite, respectively. However, a 4m-long graphite scraper is impractical due to technical problems and a high yield of the outscattered protons. Having a thin target upstream from the scraper allows a little below $2.5 \times$ $10^{9}$ and $1.9 \times 10^{11}$ protons to be scraped instantaneously, without destruction of copper, at 20 $\mathrm{TeV}$ and $2 \mathrm{TeV}$, respectively. For slow-scraping ( $>1 \mathrm{~s}$ ) by a rotatable target/scraper that is provided with a good cooling system, the total number of intercepted protons might be allowed to be much higher.

Taking into account the beam loss scenario of Section 4.0 and the SSC design requirements, ${ }^{1}$ one must design the SSC scraper system to be compatible with the scraping rates listed in Table 9.

Table 9. Maximum Scraping Rate in the Collider to Design the Scraper System.

\begin{tabular}{ccccl}
\hline $\begin{array}{c}\text { Energy } \\
(\mathrm{TeV})\end{array}$ & $\begin{array}{c}\text { Fraction of the } \\
\text { total beam }(\%)\end{array}$ & $\begin{array}{c}\text { Rate } \\
(\mathrm{p} / \mathrm{s})\end{array}$ & Time & Comments \\
\hline 2 & 1 & $1.3 \times 10^{12}$ & $0.1-1 \mathrm{~s}$ & $\begin{array}{l}\text { Beginning of } \\
\text { acceleration }\end{array}$ \\
\hline 20 & 10 & $1.4 \times 10^{10}$ & $15 \mathrm{~min}$ & $\begin{array}{l}\text { Before collisions } \\
\text { ("peak") } \\
\text { Collisions } \\
\text { ("steady state") }\end{array}$ \\
\hline \hline
\end{tabular}


Shower particles escaping a scraper can cause a quench in the downstream superconducting magnets. The previous study ${ }^{2}$ and the present calculations give the following scraping rate for 20$\mathrm{TeV}$ protons, corresponding to quench level, for a 3-m-long copper scraper positioned a few meters upstream of a superconducting element: $(1-8) \times 10^{8} \mathrm{p} / \mathrm{s}$. This depends on the halo particle distribution and the distance between the scraper and a dipole. The quench level is equivalent to about $2000 \mathrm{Mrad}$ of annual dose and $\sim 400 \mathrm{~W} /$ dipole heat load to the cold mass. ${ }^{2}$ It is clear that such a situation is unacceptable for steady state operation in the arcs; therefore, the only place for the SSC scraper system is in the west or east utility straight sections.

\subsection{INTERCEPTING COLLIMATORS}

To trap protons outscattered from the scraper, one needs to have additional collimators installed downstream. The optimum positions for such collimators can be found from the following consideration. The trajectories of outscattered protons are described using the transfer matrix parameters:

$$
x=\sqrt{\beta / \beta_{O}}\left(\cos \Delta \psi+\alpha_{o} \sin \Delta \psi\right)\left(x_{o}+\Delta x\right)+\sqrt{\beta \beta_{O}}\left(x_{o}+\Delta x^{\prime}\right) \sin \Delta \psi,
$$

where $\alpha_{O}$ and $\beta_{O}$ are Twiss parameters at the scraper positions $s_{o}, \beta$ is a $\beta$ - function at a collimator position $s, x_{O}$ and $x_{o}{ }^{\prime}$ are proton phase coordinates at the entrance to scraper (see Figure 4$), \Delta x$ and $\Delta x^{\prime}$ are the increase of proton coordinates due to scattering in the scraper material, and $\Delta \psi$ is a phase advance between $s_{O}$ and $s$. Let us assume that $x_{o}=m \sigma_{O}=\mathrm{m} \sqrt{\varepsilon \beta_{o}}$, where $\varepsilon$ is a beam emittance, and $x_{c}=n \sigma=n \sqrt{\varepsilon \beta}$ is a distance from the beam axis to a collimator jaw. Here $n, m>0$ and $n>m$. Neglecting $\Delta x$ in the above equation one can easily obtain the interception condition by the collimator:

$$
\left|\Delta x^{\prime}\right|>\sqrt{\varepsilon / \beta_{O}} !( \pm n-m \cos \Delta \psi) / \sin \Delta \psi \mid
$$

where $+n$ means the collimator installed at the positive (as a scraper) coordinate $x=n \sigma$, and $-n$ is for $x=-n \sigma$.

The optimum scraper-collimator position is determined by the following expression:

$$
\Delta \psi= \pm \operatorname{arc} \cos (m / n)+\pi k
$$

which can be supplied with a condition $n-m>\left|\Delta \mathrm{p} / \mathrm{p}\left(\eta_{o} / \sigma_{0}-\eta_{/} \sigma\right)\right|+\delta$, where $k=0,1,2 \ldots, \eta_{0}$ and $\eta$ are dispersions at the scraper and collimator positions, respectively, and $\delta \sim 1$ is an indefiniteness in distance between the closed orbit centerline and the collimator inner edge. Figure 22 shows the regions of interceptions by the collimator of protons leaked out of the scraper, 
which have an increase of the angle equal to $\delta x^{\prime}$. The results are presented for various sets of $m$ and $n$, and for both signs of $n, n>0$, (solid curves) and $n<0$ (dashed curves). One sees that the smaller $n$ is, the more efficient is the interception. To trap the protons of both signs of $\delta x^{\prime}$, two collimators are needed.

The favorable condition is to have collimators at the same side of the beam as the scraper, i.e., $x>0$, which results in the following optimal phase advances: $\Delta \psi_{1}=20-40^{\circ}$ and $\Delta \psi_{2}=300$ $320^{\circ}$. This is also true for the vertical scraping, but with $\Delta \psi_{1} \sim 40^{\circ}$ and $\Delta \psi_{2}=140-160^{\circ}$. For the scraper and collimator positioned at the different sides of the beam $(n>0, m<0)$ and for $n-m<$ 1 , the optimal phase advance is about $160^{\circ}$ (see also References 3 and 6). As shown in Figure 22, fortunately there is no strong dependence of the interception efficiency on the phase advance for a range of $\Delta \psi$. This leaves freedom to vary collimator positions to match the other requirements (see Section 9.0).

\begin{tabular}{ll|}
$\triangle m=3, n=4$ & $\times m=6, n=9$ \\
$O m=6, n=7$ & $* m=3, n=15$
\end{tabular}

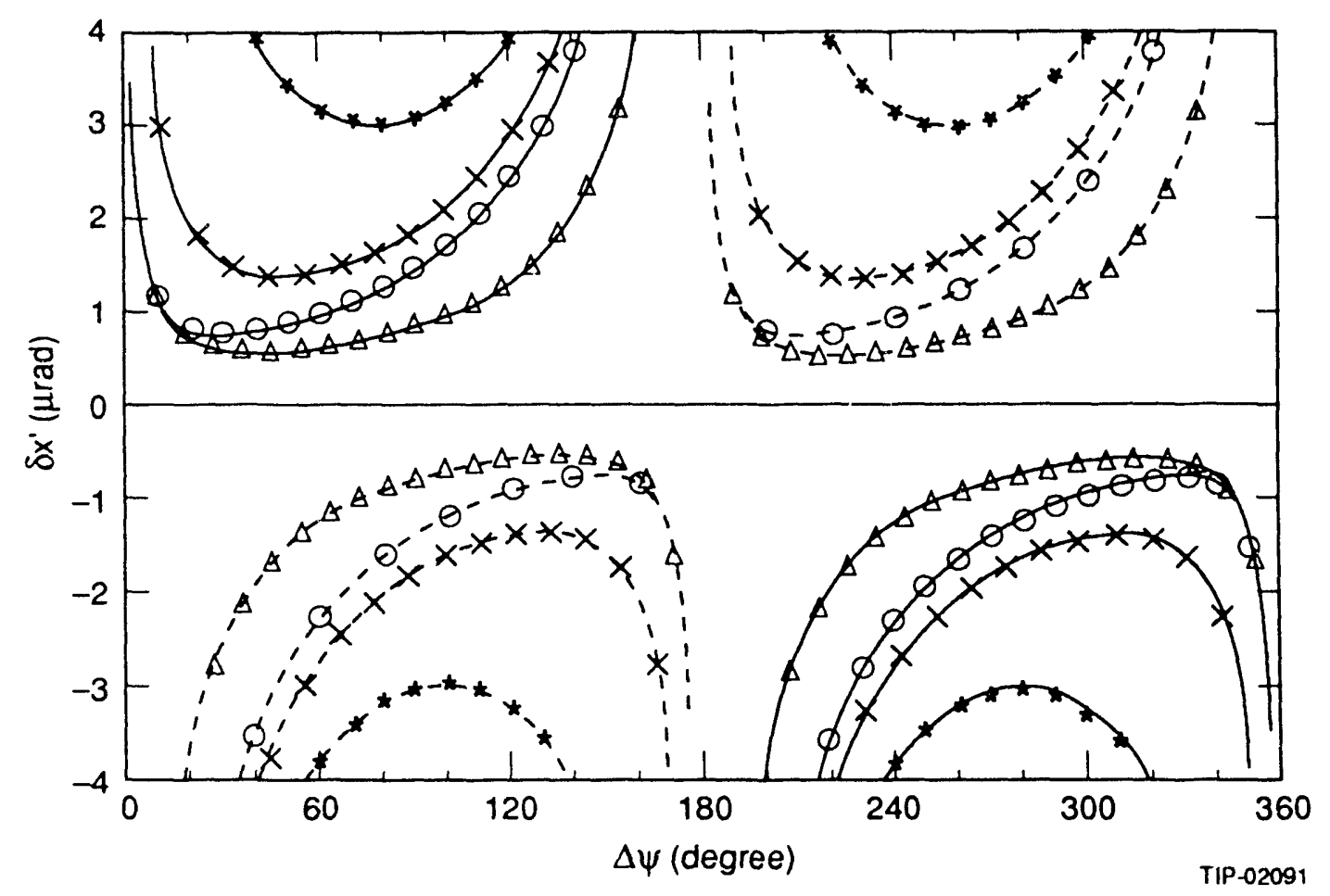

Figure 22. Efficiency of Interception by a Collimator Positioned at $n \sigma$ from the Beam Axis of Protons Leaked Out With Angle $\delta x^{\prime}$ of the Scraper, Positioned at $m \sigma$.

\subsection{SCRAPER SYSTEM IN THE EAST CLUSTER}

As mentioned in Section 5.0, to trap efficiently both off-momentum protons and particles with an increased amplitude, one needs to have a large $\beta$-function and non-zero dispersion at the scraper position. Figure 23 shows the dependence of total beam loss in the lattice $(\Delta p / p>0.01$ 
and emittance $>0.11 \pi \mathrm{mm}$-mrad) on $\beta$ for a system consisting of a $0.5-\mathrm{mm}$ tungsten target followed by an $80-\mathrm{cm}$ tungsten or $123-\mathrm{cm}$ copper scraper. A $\beta>1000 \mathrm{~m}$ would be desirable for the scraper system. The beam loss for the copper scraper is about twice that for the tungsten one at $\beta<1600 \mathrm{~m}$. The beam loss criteria for the whole machine have been checked (Figure 24), and it turns out there is no dependence of $I$ on momentum in the $0.001<\Delta p / p<0.01$ range at the given accelerator admittance. The value $0.11 \pi \mathrm{mm}$-mrad chosen above corresponds to aperture $\pm 17 \mathrm{~mm}$ at quadrupole QU2 with $\beta=2560 \mathrm{~m}$ in the east utility (or $\pm 5 \mathrm{~mm}$ in the arcs with $\beta=305 \mathrm{~m}$ ).

The non-zero dispersion option was first investigated for the west utility straight section, as in Reference 2. Unfortunately, there is not enough room there, and an interference with the injection system is a problem. To avoid this, the SSC9B lattice ${ }^{7}$ with an empty one-half standard cell in the region between dispersion suppressor and the utility has been explored. The $\beta$-function there can be made as high as $250 \mathrm{~m}$ with a dispersion $\eta=2.5 \mathrm{~m}$. Given this, only an inefficient, very simple system, consisting of one scraper and one intercepting collimator downstream, can be installed there. A few full-scale Monte Carlo calculations have been done to optimize the scheme. Even in the best case, the resulting beam loss in the lattice is as high as $20 \%$ per one proton trapped with a target/scraper system, and energy deposition in the downstream superconducting elements (Figure 25) exceeds the quench level at only a few by $10^{8}$ $\mathrm{p} / \mathrm{s}$ scraping rate. (Remember that this corresponds to an extremely high accumulated dose and about $400 \mathrm{~W}$ heat load to the cold mass.)

So finally the East Cluster has been chosen as a place for the whole SSC scraper system. The SSC10F lattice, which allows installation of a very effective scraper system for the whole SSC cycle, has been calculated by Al Garren. ${ }^{7}$ At the position appropriate for the scraper, $\beta_{x}=1200 \mathrm{~m}$ and dispersion $\eta=-4 \mathrm{~m}$. The schematic view of the east utility is shown in Figure 26, where $s$ is a distance to the element upstream from the beginning of the straight section (QSF) and is equal to $s=s(\mathrm{SYNCH})-1350 \mathrm{~m}$. The rest of the paper deals with this structure.

The optimum values of the proposed scraper system parameters are listed in Tables 10 and 11. The main results of our studies are presented below. The dogleg structure consists of three simple bump-magnets BM1-BM3 with an aperture of about $40 \mathrm{~mm}$; it provides a complete interception of neutral and low-energy charged particles out of the scrapers. Moreover, such a scheme offers a chance to later trap the off-momentum protons by adding the transverse angle $\sim \phi$ $\Delta p / p$, where $\phi$ is a bump-bending angle. 


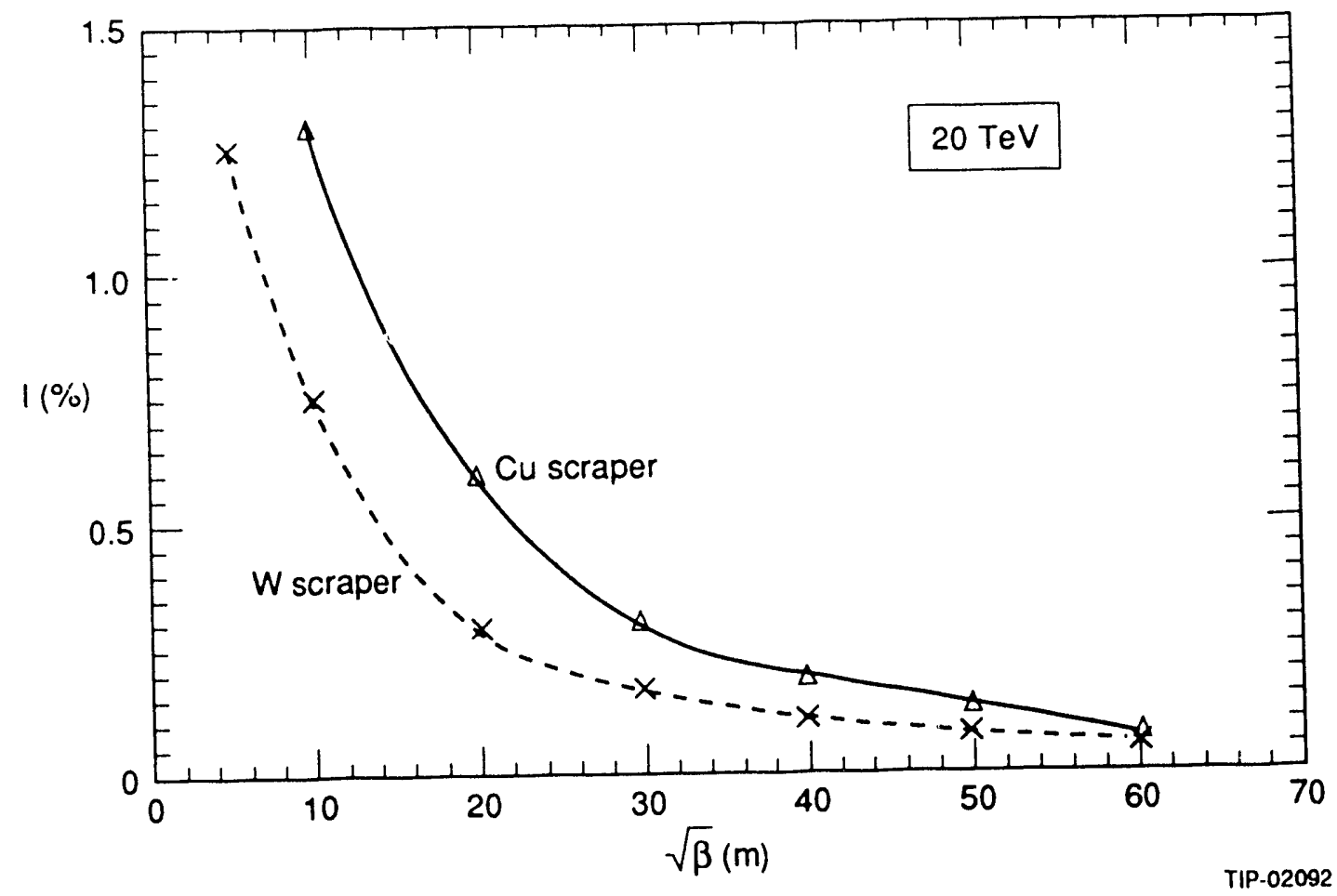

Figure 23. Total Beam Loss in the SSC Lattice Per One 20-TeV Proton Scraped by Tungsten or Copper Scraper Versus $\beta$-Function at the Scraper Position.

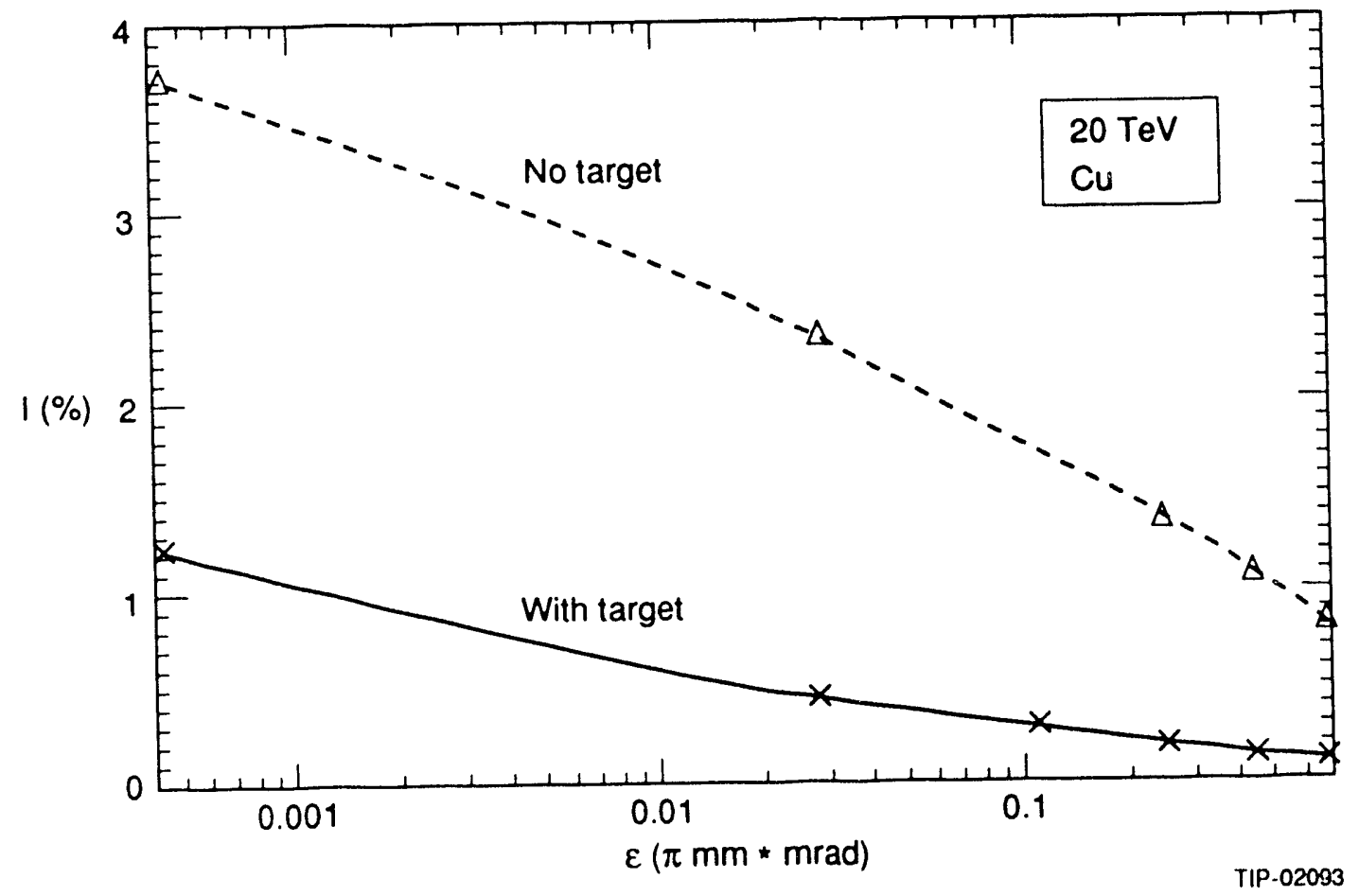

Figure 24. Total Beam Loss in the SSC Lattice Per One 20-TeV Proton Scraped by Copper Scraper With and Without Tungsten Target as a Function of the Accelerator Admittance. 


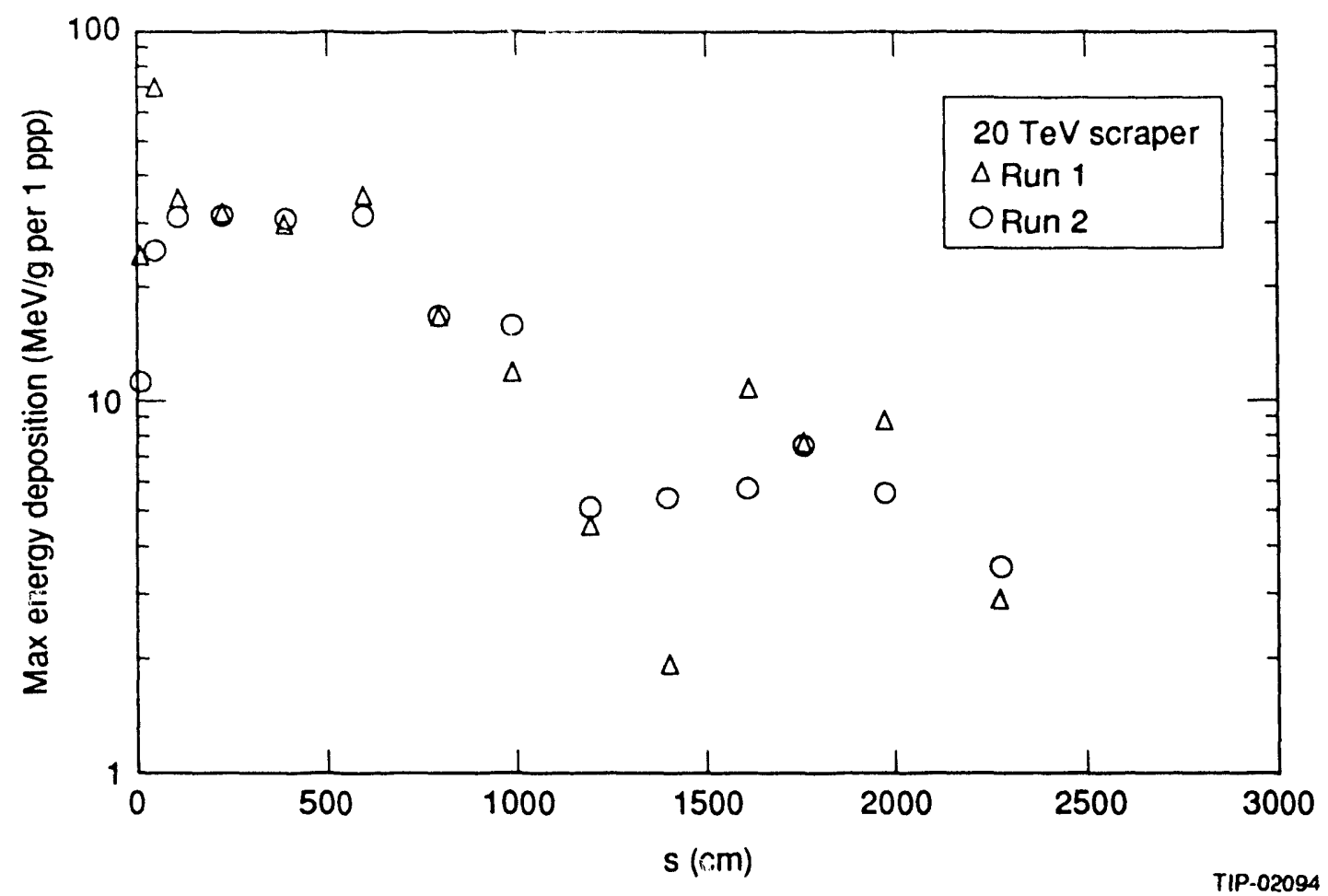

Figure 25. Maximum Energy Deposition (Per One Scraped Proton) in the Superconducting Coils of Two Dipoles $7 \mathrm{~m}$ Downstream of the $3 \mathrm{~m}$ Copper Scraper (With a Target) With Additional Collimator With ID = $34 \mathrm{~mm}$, Length $=3 \mathrm{~m}$ (run 1) and ID $=20 \mathrm{~mm}$, Length $=5 \mathrm{~m}$ (Run 2) Just Before the First Dipole.

Table 10. Scraper System Parameters: Target Scrapers and Collimators.

\begin{tabular}{ccccccc}
\hline No. & Element & $S(\mathrm{~m})$ & $L(\mathrm{~m})$ & Material & $x(\mathrm{~mm})$ & $y(\mathrm{~mm})$ \\
\hline 1 & TAR-X & 476 & 0.0005 & $W$ & $+(.80-3)$ & \\
2 & SCR-X & 476 & 0.8 & $W$ & $+(.85-3)$ & \pm 20 \\
& & & $(1.2)$ & $(\mathrm{Cu})$ & & \\
3 & TAR-Y & 480 & 0.0005 & $W$ & $+(.60-3)$ & \\
4 & SCR-Y & 480 & 0.8 & $W$ & \pm 20 & $+(.65-3)$ \\
& & & $(1.2)$ & $(\mathrm{Cu})$ & & \\
5 & C1/C2 & 923 & $2-3$ & $\mathrm{Fe}$ & $+(.5-10)$ & $+(.5-10)$ \\
& & & & & $-(5-10)$ & $-(5-10)$ \\
6 & $\mathrm{C} 3 / \mathrm{C} 4$ & 1270 & $2-3$ & $\mathrm{Fe}$ & $+(.5-10)$ & $+(.5-10)$ \\
\hline \hline
\end{tabular}




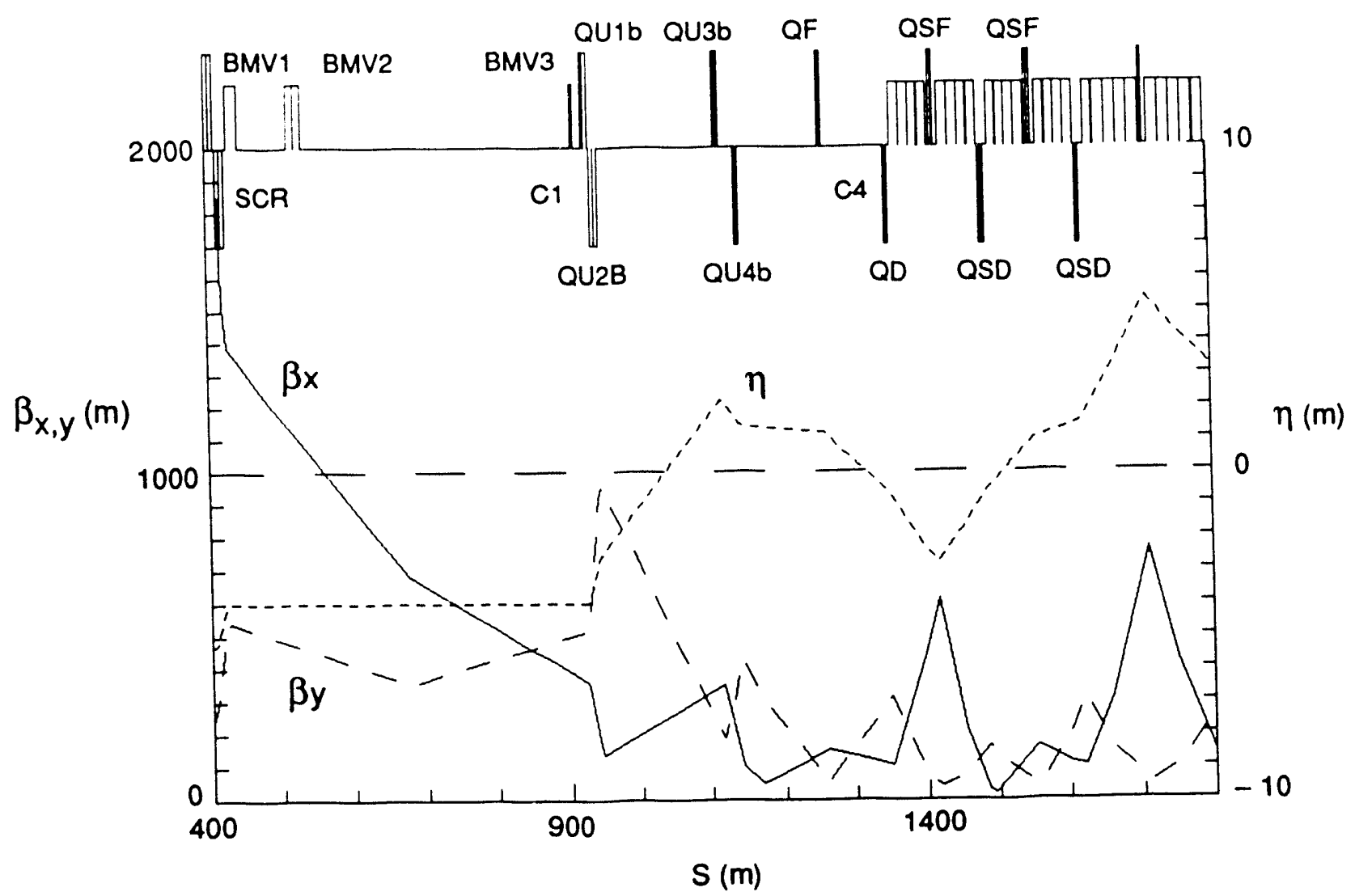

Figure 26. Lattice Functions and Schematic Magnet Layout for an East Utility Straight Section Scraper System.

Table 11. Scraper System Parameters: Dogleg Magnets.

\begin{tabular}{ccccc}
\hline No. & Element & $S(\mathrm{~m})$ & $L(\mathrm{~m})$ & $\mathrm{B}(\mathrm{kG})$ \\
\hline \multicolumn{5}{c}{ Asymmetric Dogleg } \\
\hline 1 & $\mathrm{BM} 1$ & 426.5 & 15 & -20 \\
2 & $\mathrm{BM} 2$ & 521.0 & 20 & +20 \\
3 & $\mathrm{BM3}$ & 916.5 & 5 & -20 \\
\hline \multicolumn{6}{c}{ Dogleg with Lambertson } \\
\hline 1 & $\mathrm{BM} 1$ & 428.5 & 27.5 & -17.1 \\
2 & $\mathrm{BM} 2$ & 628.0 & 94.0 & 10.0 \\
3 & $\mathrm{BM3}$ & 894.0 & 27.5 & -17.1 \\
\hline \hline
\end{tabular}


Figures 27-31 show the calculated trajectories of protons outscattered of the scraper. Particles downstream of the scraper and dogleg can be intercepted by two sets of collimators:

1. $\mathrm{C} 1$ (horizontal, $\Delta \psi_{1}=40^{\circ}$ ) and $\mathrm{C} 2$ (vertical, $\Delta \psi_{2}=65^{\circ}$ ) just upstream of QU1b; $n=m+0.6+\delta(\sim m+1.6$ at $20 \mathrm{TeV})$.

2. C3 (horizontal, $\Delta \psi_{1}=300^{\circ}$ ) and $\mathrm{C} 4$ (vertical, $\Delta \psi_{2}=150^{\circ}$ ) downstream of $\mathrm{QF}$ quadrupole; $n=m+1.4+\delta(\sim m+2.4$ at $20 \mathrm{TeV})$.

Without a dogleg system, there is no way to trap the secondary particles with $\alpha \sim 0$ in the straight section: most of these are lost in the very first superconducting dipoles downstream of the utility (Figure 27).

Two possible configurations for the dogleg have been studied (Table 11):

1. a simple, inexpensive asymmetric system dedicated only to scraper system functions, or

2. a dogleg with a Lambertson magnet that matches the requirements of the SFT experiment, which can be used both for a significant increase in scraping efficiency and for slow extraction of the beam via the use of a bent crystal.

It turns out that although the second system is a better catcher of secondaries, the long-range beam loss in the lattice is almost the same. So, the final choice should be made taking into account all other considerations (cost, SFT experiment schedule, etc.). Results below are presented for the simple first dogleg system.

Three schemes for the beam gymnastics have been checked:

1. a horizontal dogleg away from the ring center (Figure 29);

2. a horizontal dogleg toward the ring center (Figure 30);

3. a vertical dogleg (Figure 31).

The interception efficiency for all three cases is very similar, although case (b) leaves more flexibility because the collimators are installed at the same side as the scraper $(m, n>0)$. To be compatible with the dogleg system with a Lambertson magnet, all the results below are given for case (a). Figures 32-38 present the results of the intercepting collimators $\mathrm{C} 1-\mathrm{C} 4$ efficiency studies. Distributions of protons to be lost in the machine in coordinates and angles are shown in Figures 32 and 33 for the collimator $\mathrm{C} 1 / \mathrm{C} 2$ position, and the phase-space portraits of these are presented in Figures 34 and 35 for both the collimator pairs. Closing of $\mathrm{C} 1$ collimator jaws up to $x= \pm 5 \mathrm{~mm}$ results in the cut of the above distribution (Figure 36). Dependence of the total beam loss in the machine on the distance of collimators $\mathrm{C} 1$ and $\mathrm{C} 2$ jaws from the beam center $\mathrm{D}$ is shown in Figure 37 for horizontal plane scraping. Results are given for simultaneous symmetrical movement of both jaws in the horizontal (C1) and vertical (C2) planes. The similar 


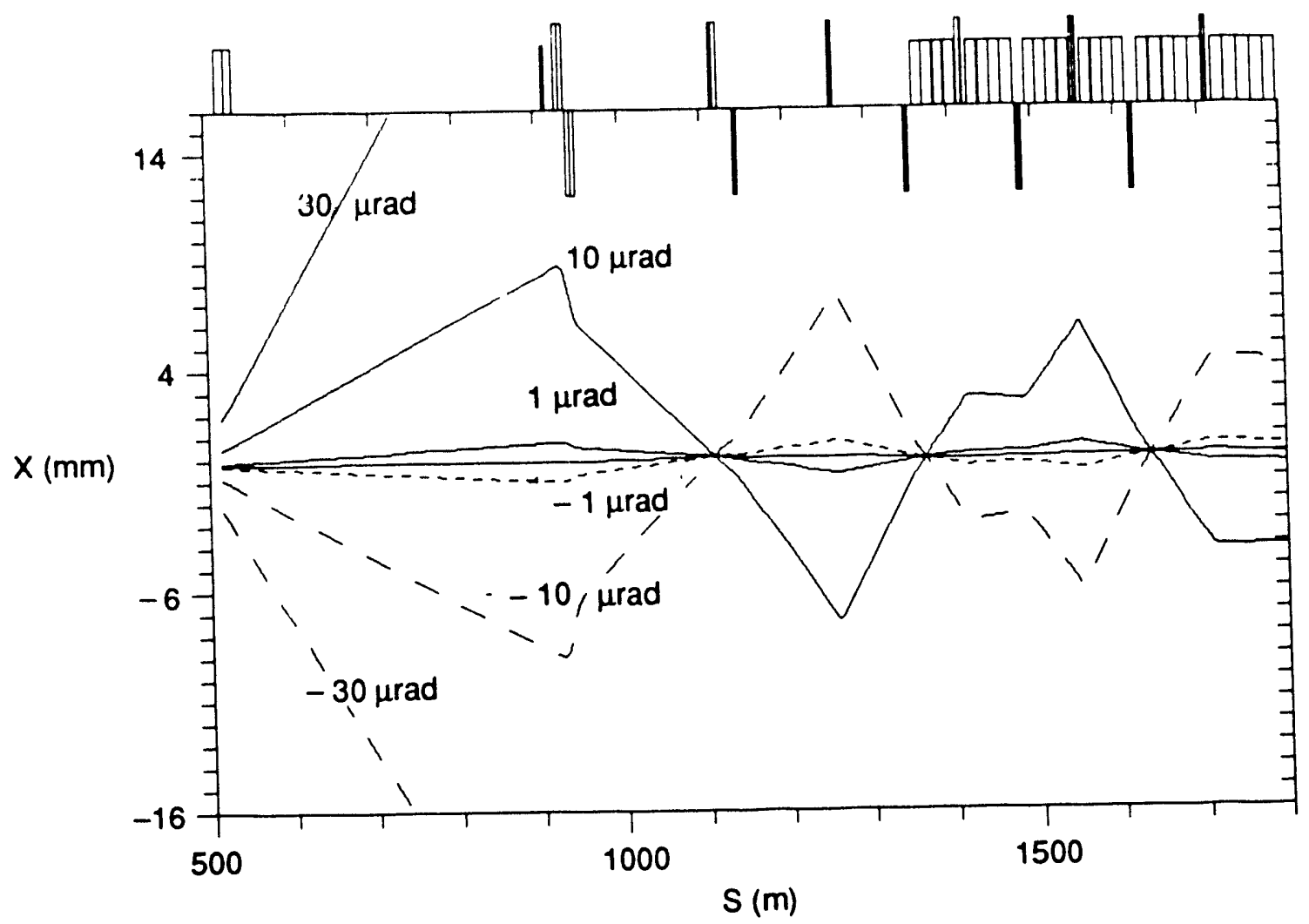

Figure 27. Trajectories of Protons Elastically Scattered Out of Scraper.

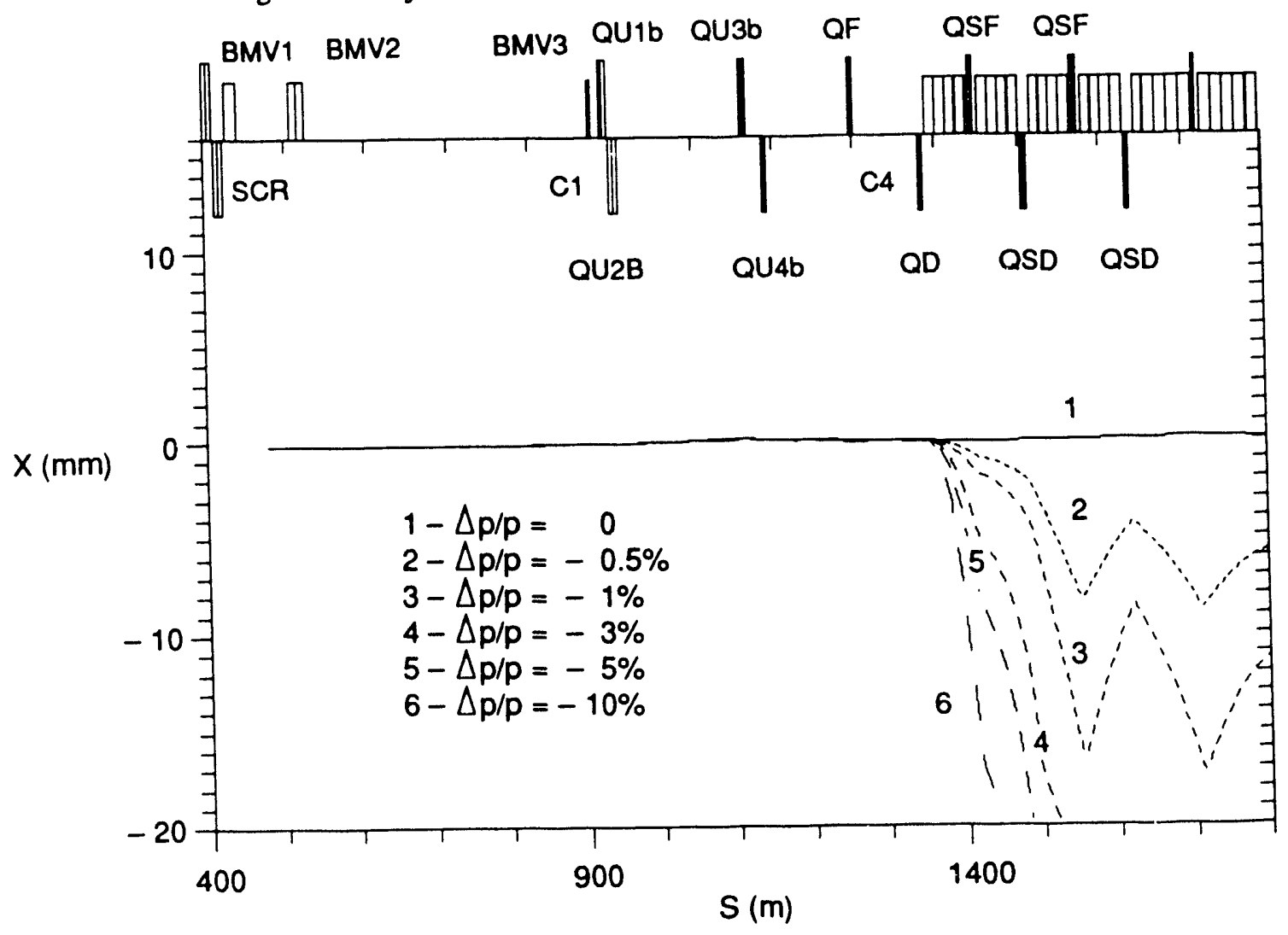

Figure 28. Trajectories of Protons Which Exit the Scraper With Various Momentum Change in the East Utility Straight Section. 


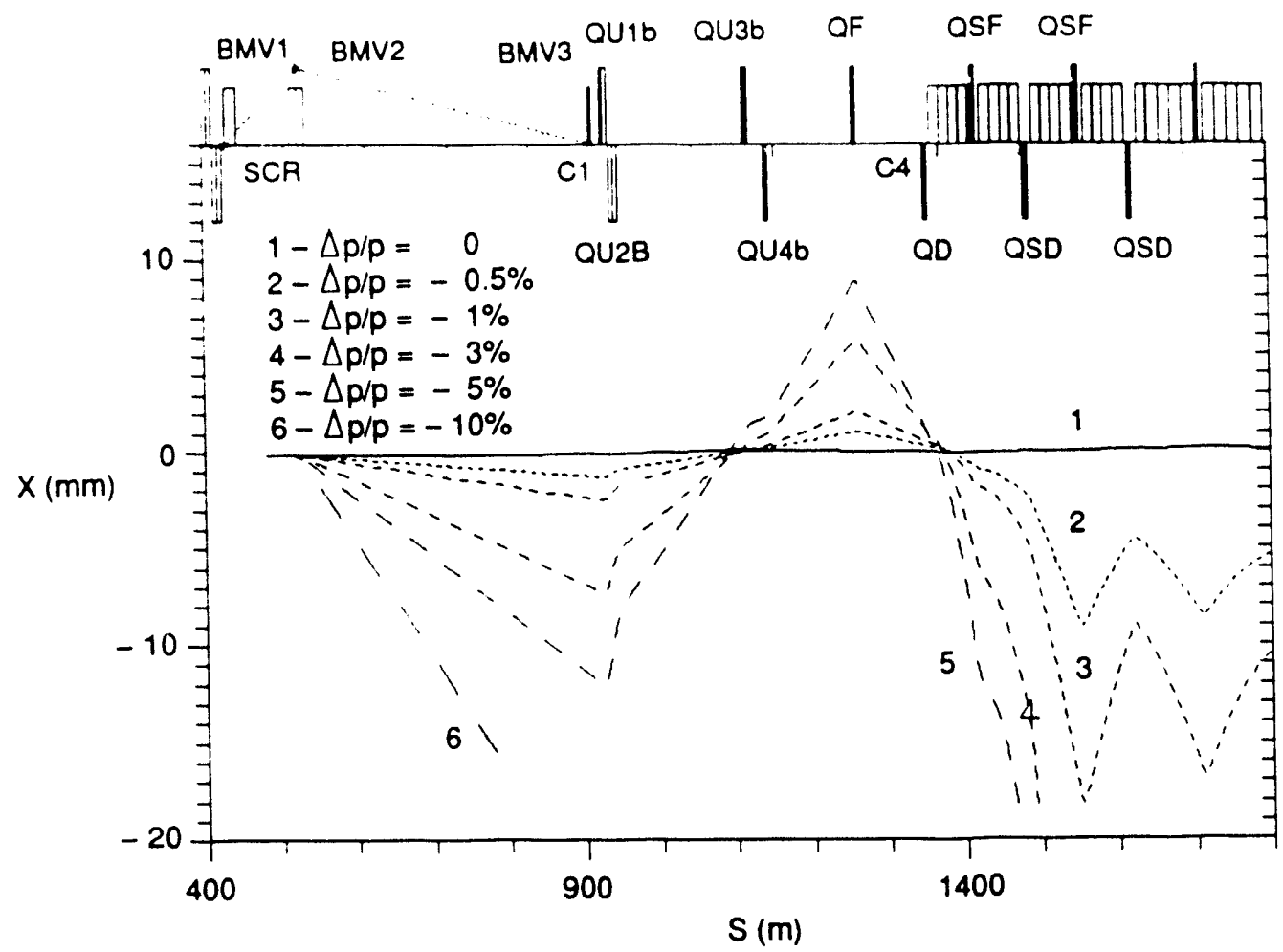

Figure 29. Trajectories of Protons Which Exit the Scraper With Various Momentum Change in the East Utility Straight Section, With a Horizontal Dogleg Out of the Ring Center.

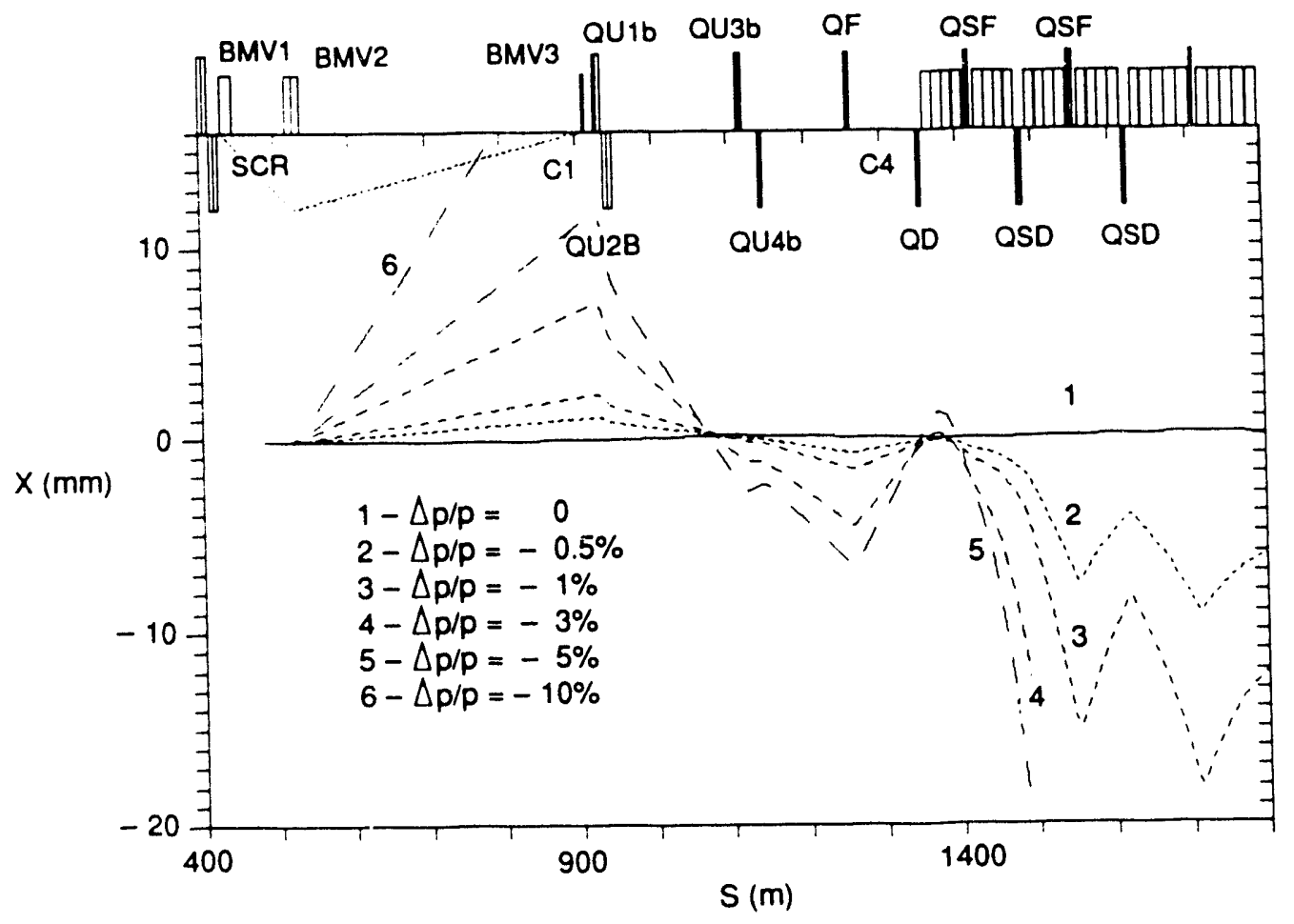

Figure 30. Trajectories of Protons Which Exit the Scraper With Various Momentum Change in the East Utility Straight Section, With a Horizontal Dogleg to the Ring Center. 


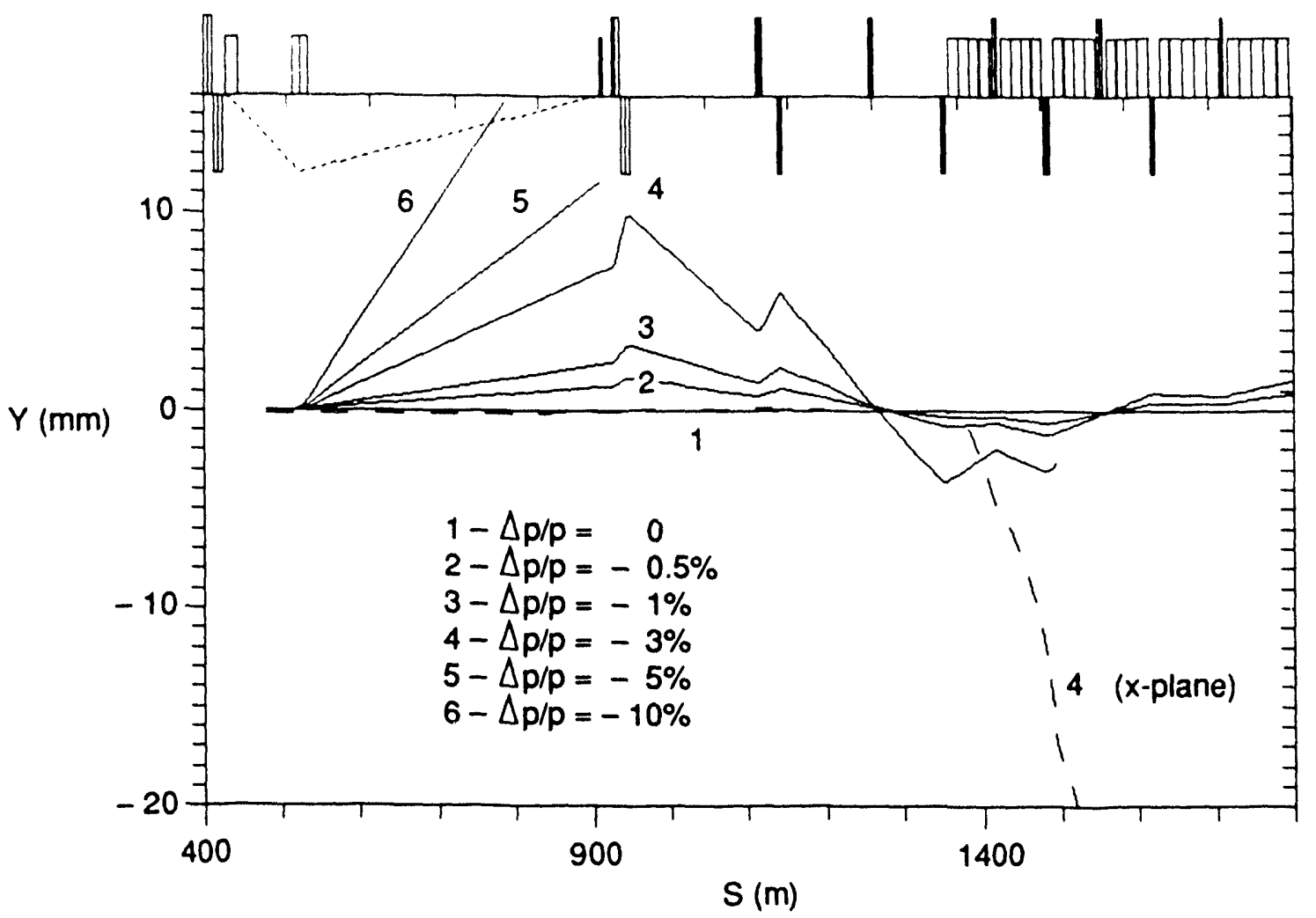

Figure 31. Trajectories of Protons Which Exit the Scraper With Various Momentum Change in the East Utility Straight Section, With a Vertical Dogleg.

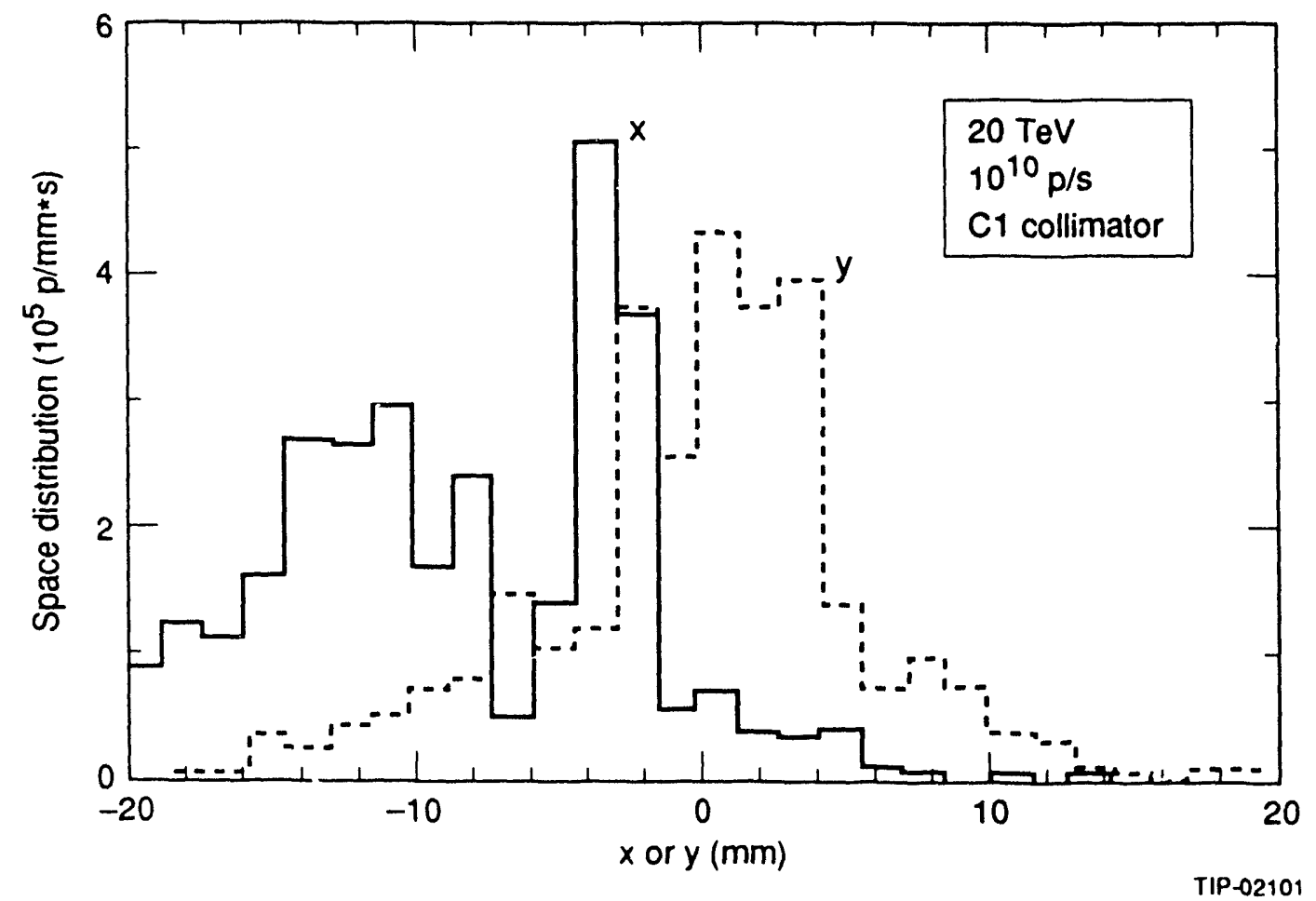

Figure 32. Horizontal and Vertical Distributions of Protons to be Lost on the Machine Aperture, at Collimator C1 Position. 


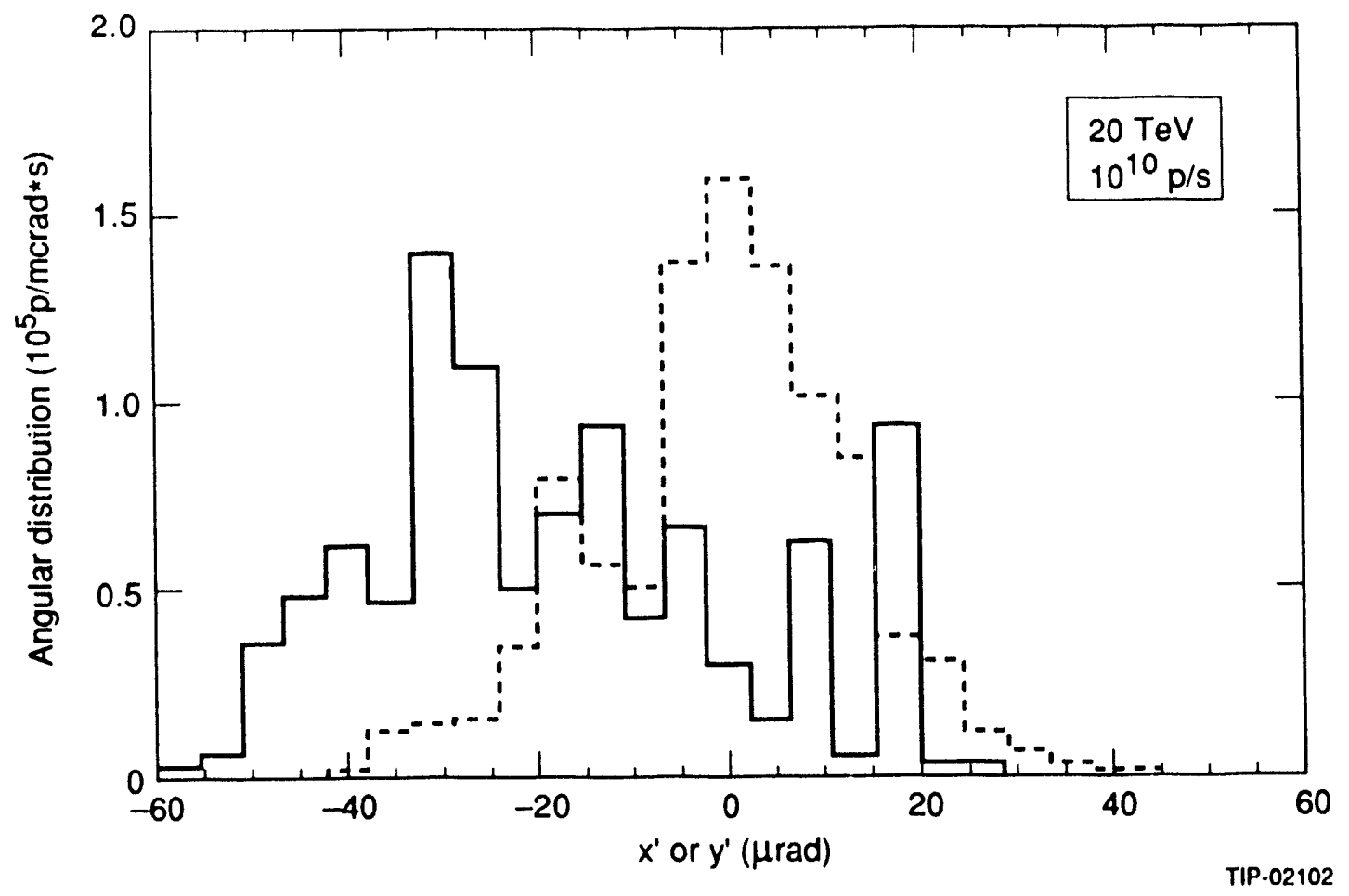

Figure 33. Angular Distributions of Protons to be Lost on the Machine Aperture, at Collimator C1 Position.

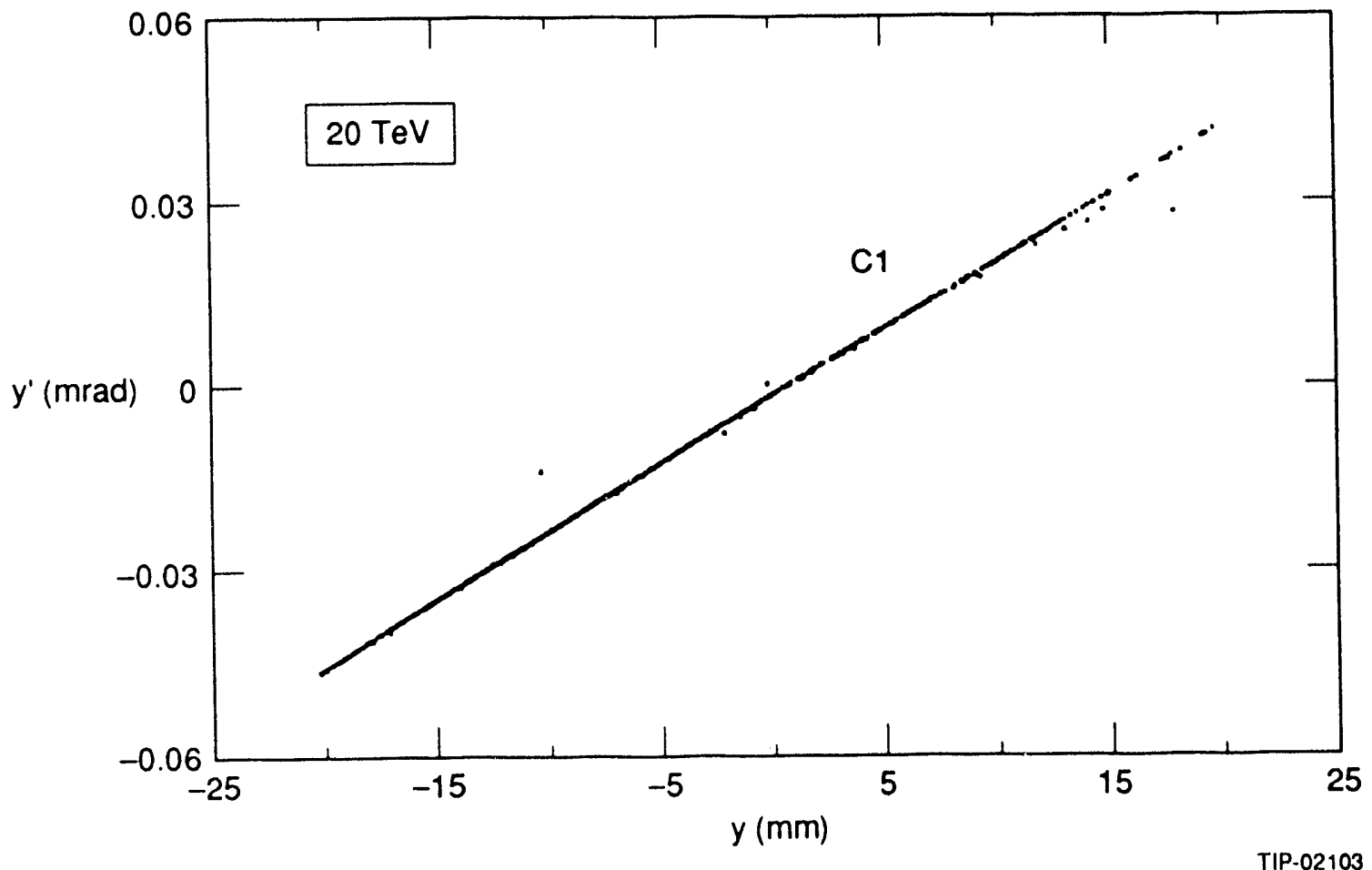

Figure 34. Vertical Plane Phase Space Portrait for Protons to be Lost in the Machine, at Collimator C1/C2 Position. 


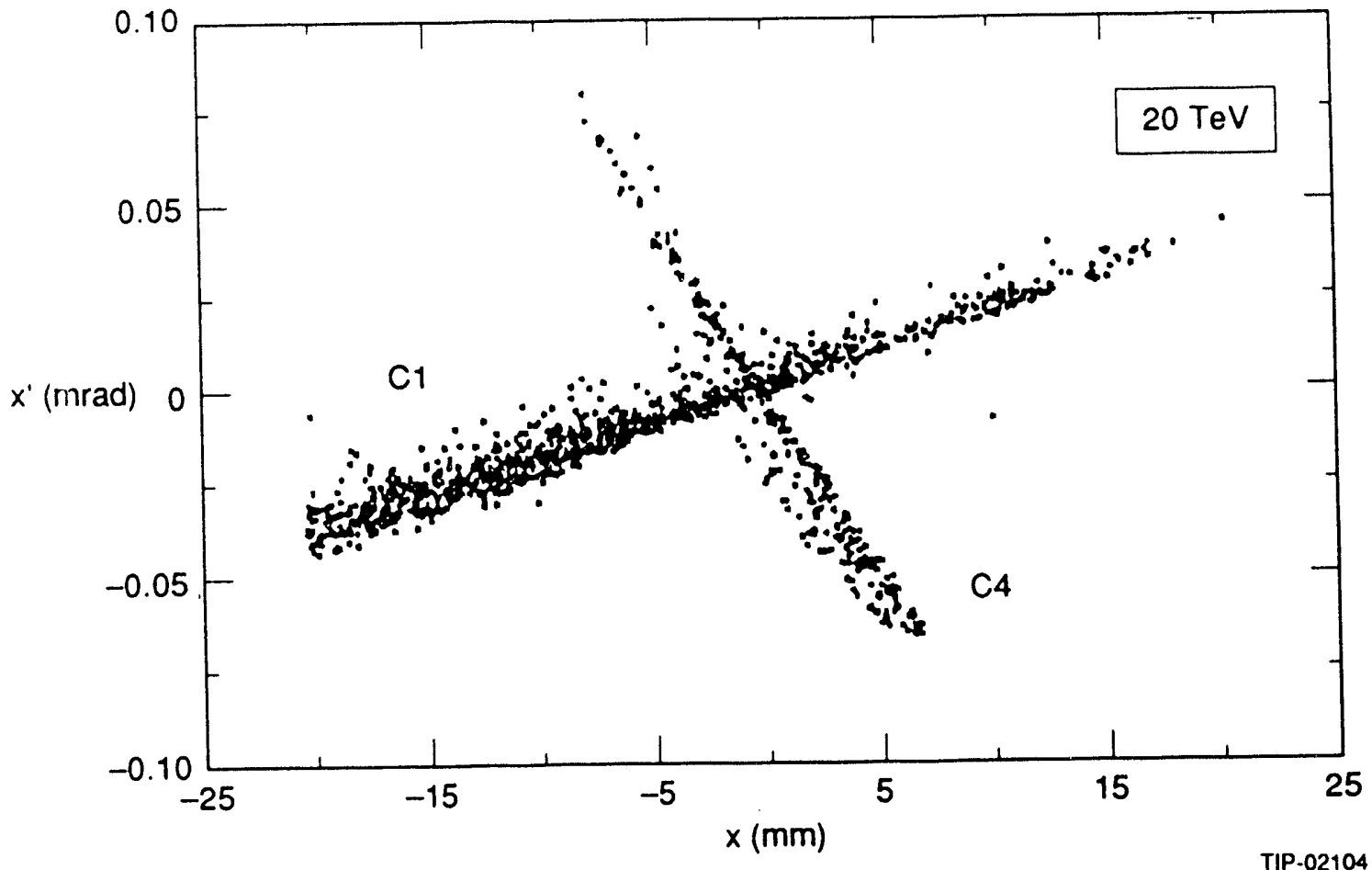

Figure 35. Phase Space Portrait in Horizontal Plane of Protons to be Lost in the Machine, at Collimators C1/C2 and $\mathrm{C} 3 / \mathrm{C} 4$ Positions.

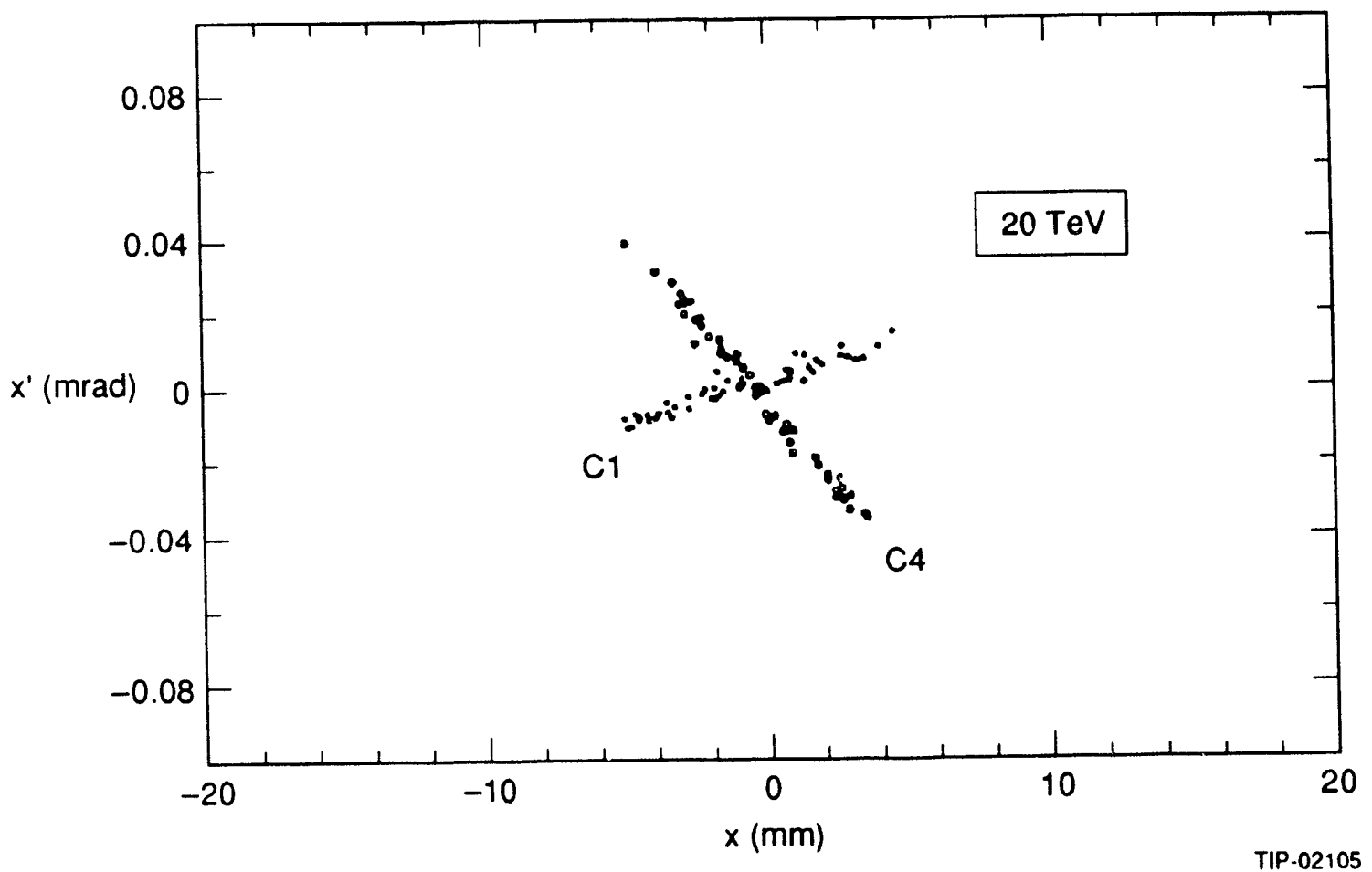

Figure 36. Phase Space Portrait in Horizontal Plane of Protons to be Lost in the Machine, at Collimators $\mathrm{C} 1 / \mathrm{C} 2$ and $\mathrm{C} 3 / \mathrm{C} 4$ Positions, With Collimator C1 Aperture Equal to $\pm 5 \mathrm{~mm}$. 


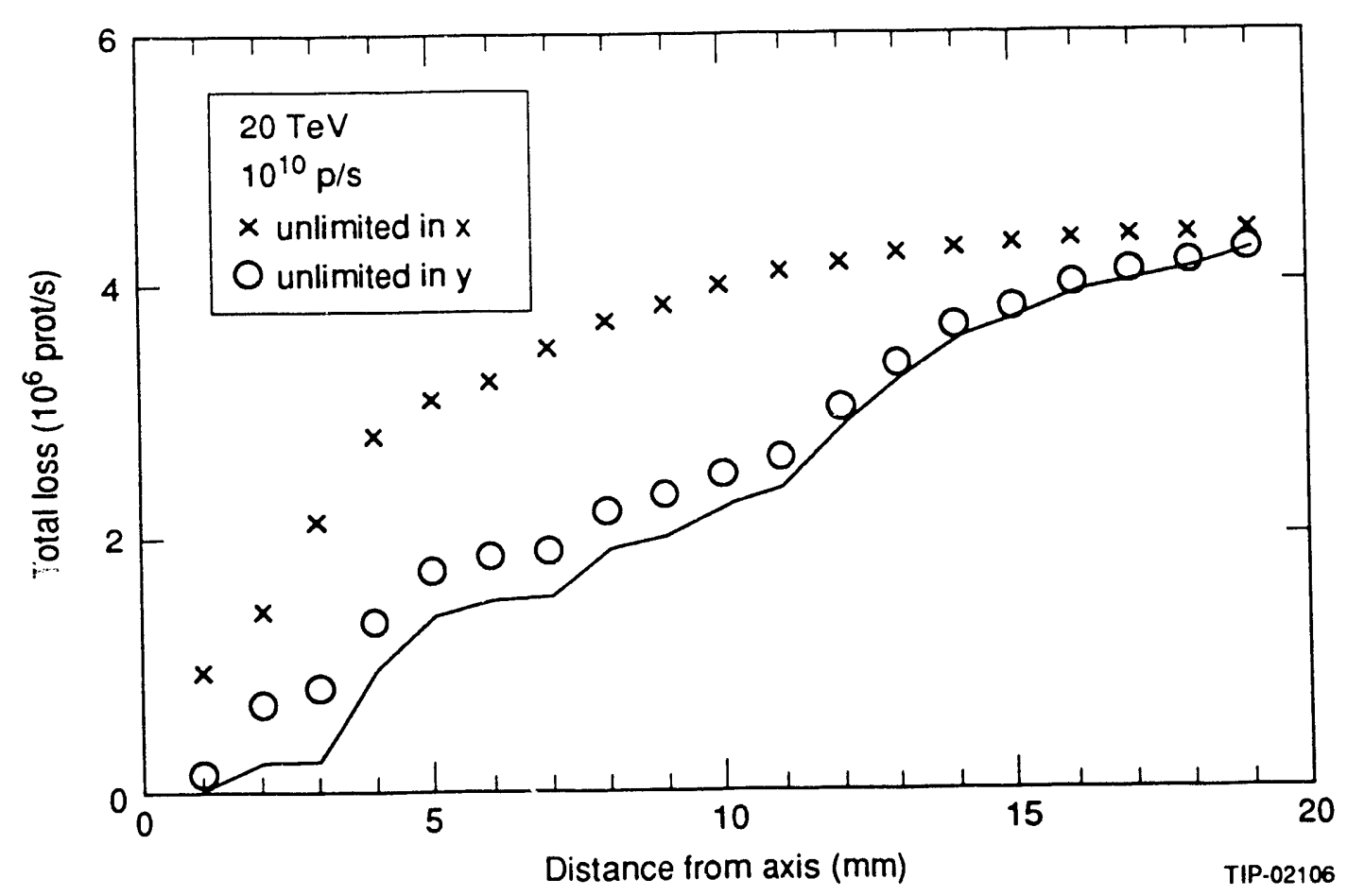

Figure 37. Total Beam Loss in the SSC Lauirs for $10^{10}$ Protons Per Second Scraping Rate at Scraper SCR-X (the First 15 min at Full Energy) Versus $\mathrm{C} 1 / \mathrm{C} 2$ Aperture.

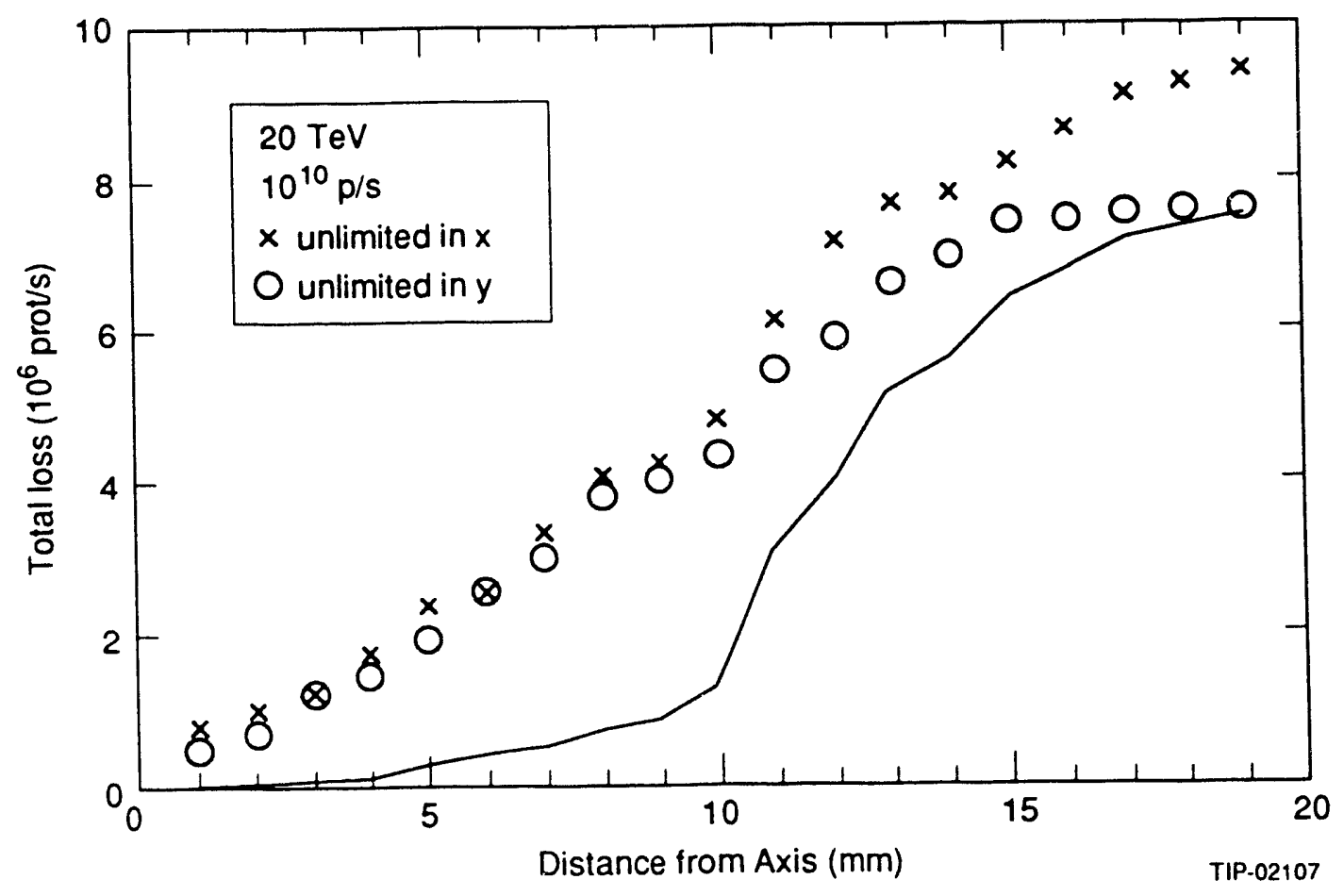

Figure 38. Total Beam Loss in the SSC Latuice for $10^{10}$ Protons Per Second Scraping Rate at Vertical Scraper SCR-Y (the First 15 min at Full Energy) Versus C1/C2 Aperture. 
dependence is seen for the vertical scraping (Figure 38). Note that at $D>10 \mathrm{~mm}$ outscattered protons with large $\Delta p / p$ determine the integral beam loss. Protons with the larger amplitude dominate for smaller $D$.

The calculated beam loss density in the utility straight section and in the first downstream lattice elements are shown in Figures 39-41 for the following cases: without beam gymnastics, with a simple dogleg system, and with additional collimators. Results are given for the peak scraping rate $10^{10} \mathrm{p} / \mathrm{s}$. One sees that the proposed measures clean up the straight section and reduce beam loss in the "hottest" lattice elements by almost 10 times with the bump magnet system (Figure 40) and about $\sim 100$ times with the additional collimators C1/C2 and C3/C4 (Figure 41).

Details of the beam loss distribution in the first superconducting dipoles and quadrupoles with and without protective measures are shown in Figure 42. Results of the full-scale energy deposition calculations in the "hottest" superconducting elements, taking into account both the above beam loss and the secondaries produced at collimators during the additional beam cleaning procedure, are presented in Figures 43 and 44 . The maximum energy deposition density in the coils and the additional heat load to the cold mass are well below the tolerable levels, ${ }^{2}$ even at the peak scraping rate.

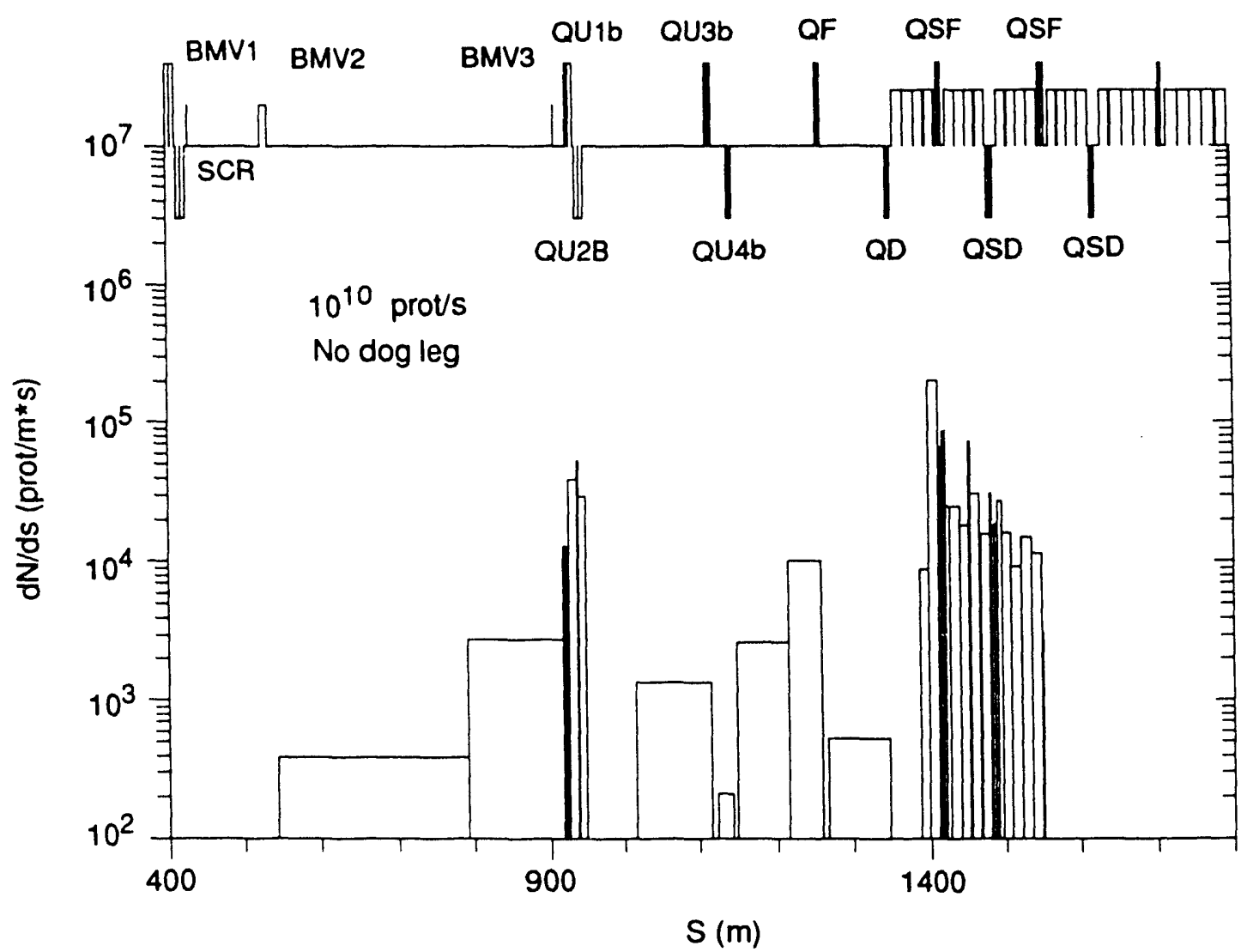

Figure 39. Beam Loss Density Distribution in the East Utility Downstream of the Scrapers for a Scraping Rate of $10^{10}$ Protons Per Second. 
It was found that the protective efficiency of the above system at injection energy is lower compared to the full energy. But with a lower energy deposition at $2 \mathrm{TeV}$ and higher tolerable levels in the superconducting coils, there is about the same safety factor even at the assumed injection scraping rate of $1.3 .10^{12}$ protons per $0.1-1$ second (Table 9).

We suggest the following simple scheme of short bump magnets (Table 12), which allows control of the beam displacement at the scraper position in the regions $\pm 3 \mathrm{~mm}$ and $\pm 15 \mu \mathrm{rad}$.

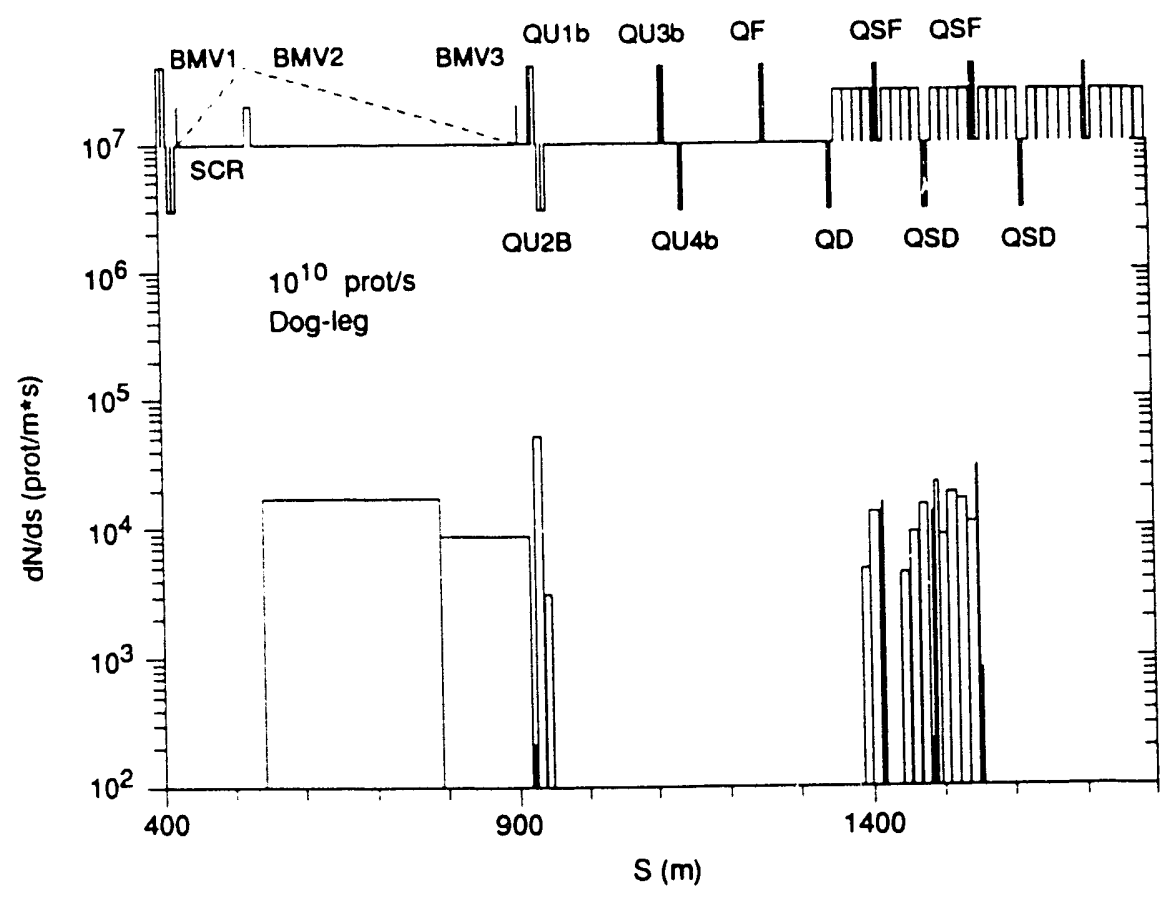

Figure 40. Beam Loss Density Distribution in the East Utility Downstream of the Scrapers for a Scraping Rate of $10^{10}$ Protons Per Second, With the Bump Magnet System.

Table 12. Scraper System Parameters: Lead Up Magnets.

\begin{tabular}{ccccc}
\hline No. & Element & $S(\mathrm{~m})$ & $L(\mathrm{~m})$ & $\mathrm{B}(\mathrm{kG})$ \\
\hline 1 & $\mathrm{BH} 1$ & 213.5 & 1 & 9 \\
2 & $\mathrm{BV} 1$ & 240.5 & 1 & 9 \\
3 & $\mathrm{BH} 2$ & 260.5 & 1 & 6 \\
4 & $\mathrm{BV} 2$ & 320.5 & 1 & 6 \\
5 & $\mathrm{BV3}$ & 880.0 & 1.5 & 10 \\
6 & $\mathrm{BV} 4$ & 969.4 & 1 & 6 \\
7 & $\mathrm{BH3}$ & 1057.2 & 2 & 10 \\
8 & $\mathrm{BH} 4$ & 1109.2 & 1 & 6 \\
\hline \hline
\end{tabular}




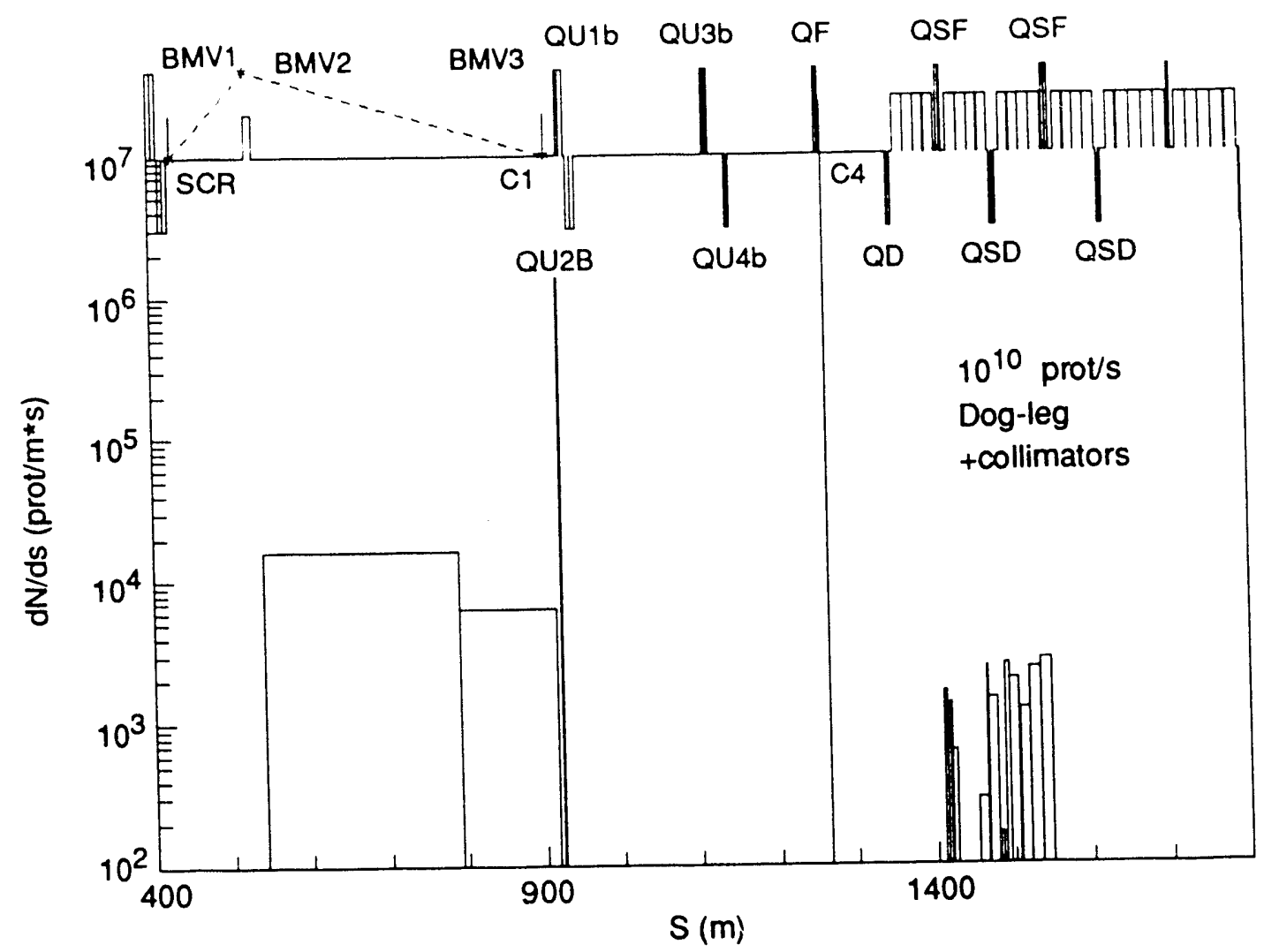

Figure 41. Beam Loss Density Distribution in the East Utility Downstream of the Scrapers for a Scraping Rate of $10^{10}$ Protons Per Second, With the Bump Magnet System and With the Additional Collimators.

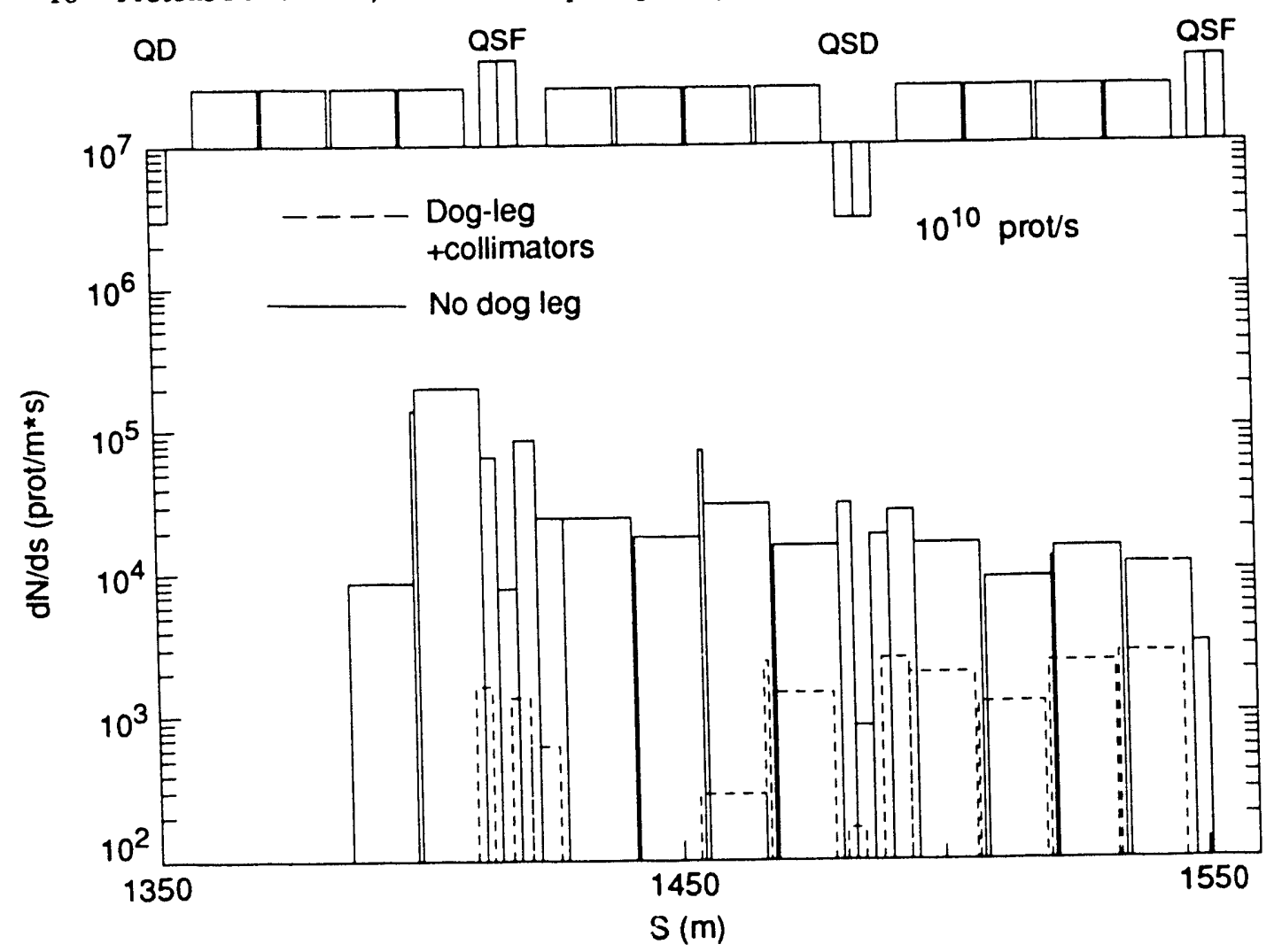

Figure 42. Beam Loss Densiiy Distribution in the Dispersion Suppressors. 


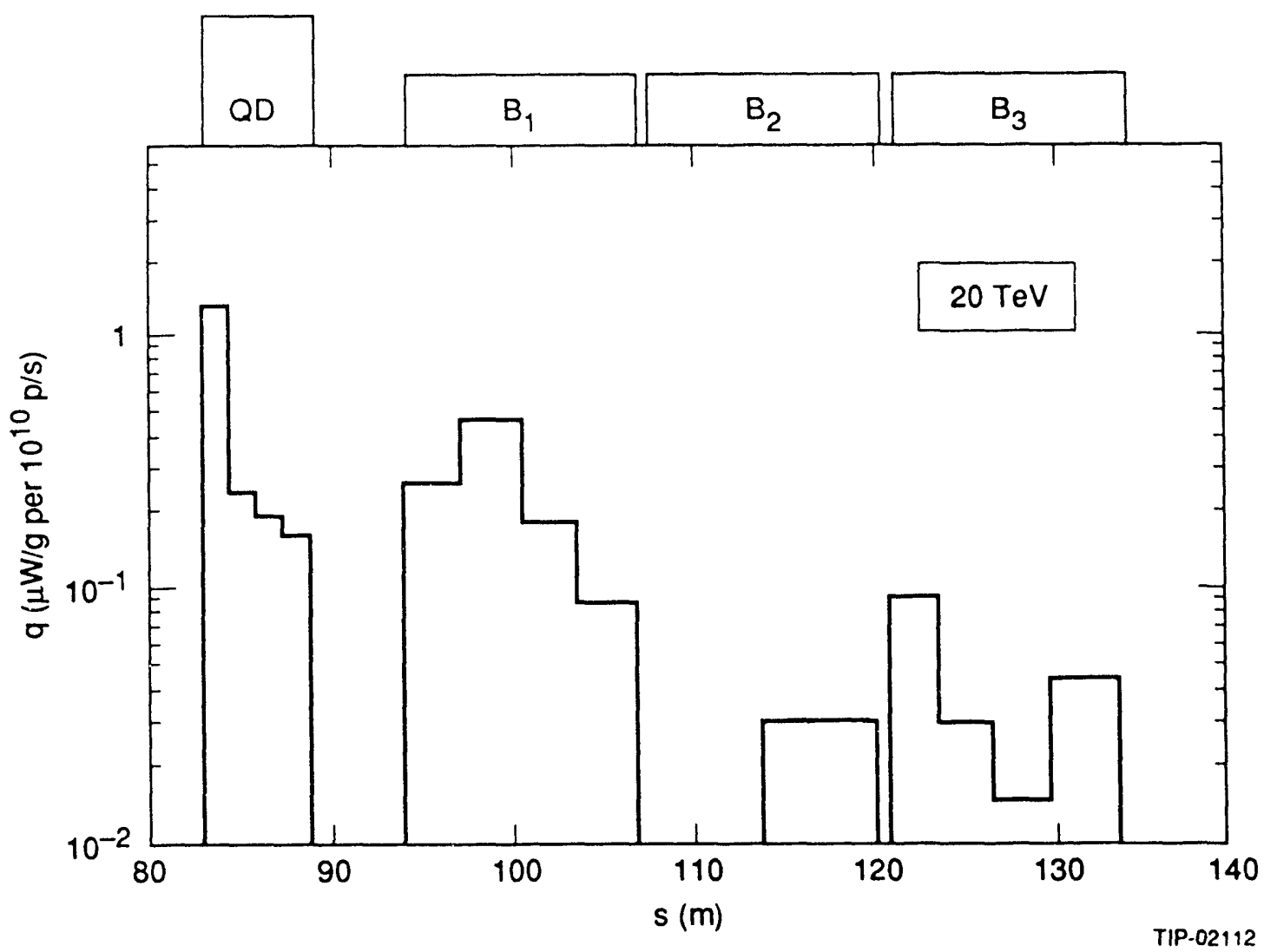

Figure 43. Maximum Energy Deposition Density in the Superconducting Coils of the Dispersion Suppressor Elements as a Function of Distance from Collimator C3/C4.

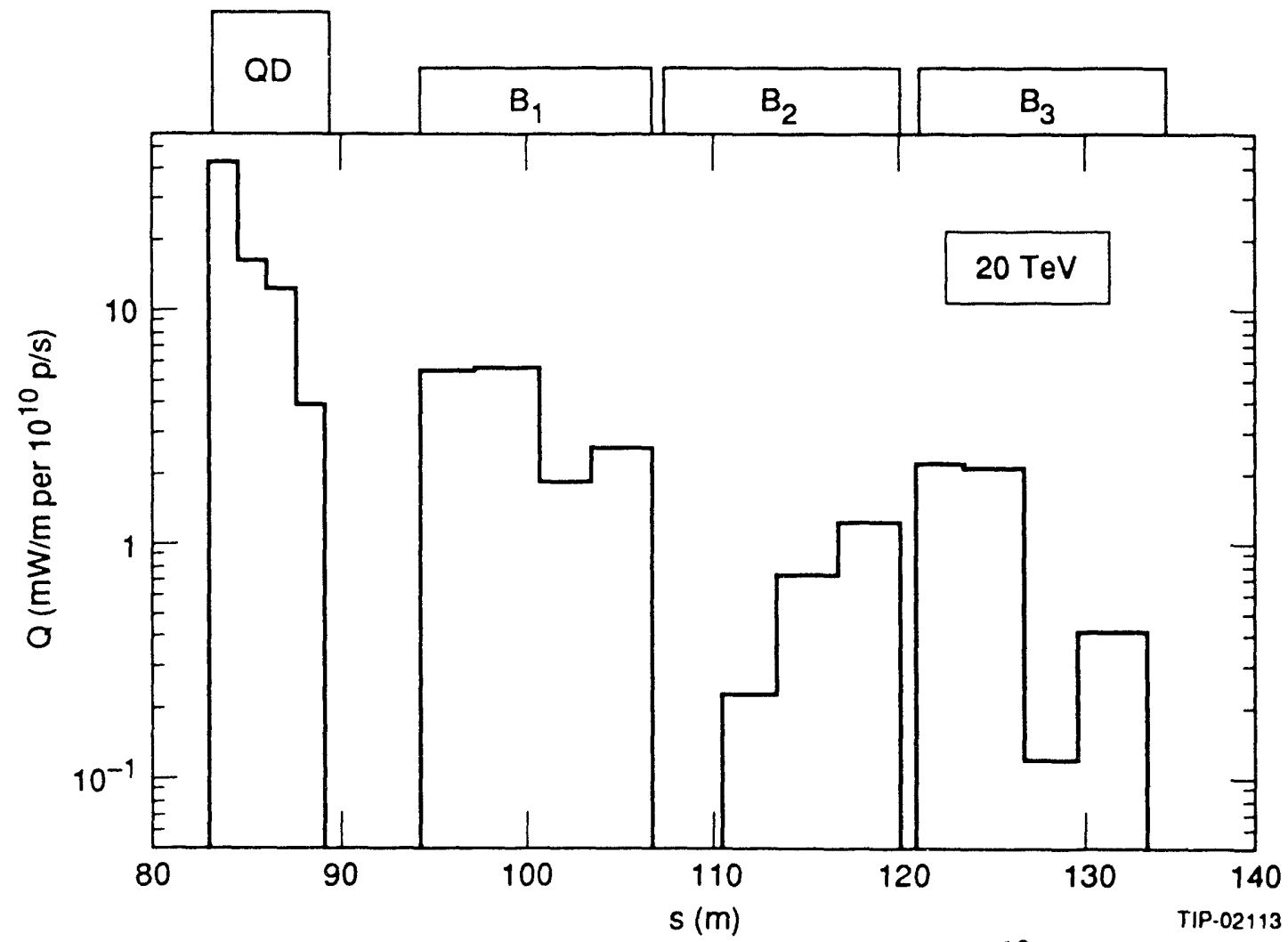

Figure 44. Power Deposited Per $1 \mathrm{~m}$ of the Dispersion Suppressors for $10^{10} \mathrm{p} / \mathrm{s}$ Scraping Rate. 


\subsection{SCRAPER: COOLING, SHIELDING, RADIOACTIVATION}

As pointed out above, the scraper itself has to be designed to be compatible with a high scraping rate and corresponding hard radiation environment. Material integrity is influenced by maximum energy deposition, longitudinal and lateral distributions of which are presented in Figures 45 and 46 for all the parameters found in the previous section. The maximum is reached at a $25-\mathrm{cm}$ depth for copper and at about $17 \mathrm{~cm}$ for tungsten scrapers for the considered case with a 0.5-mm tungsten target. In the transverse direction, for the same case, the maximum occurs at a distance of about $0.1 \mathrm{~mm}$ from the inner edge. The total beam energy absorbed at $20 \mathrm{TeV}$ in such a scraper and in the first few centimeters of the surrounding steel (Figure 10) is shown in Figure 47 as a function of scraper length and is needed to determine parameters of the cooling system.

Energy deposition and accumulated dose in the "hottest" regions are given in Table 13 for the horizontal scraper, the bump magnets of the dogleg system, and two superconducting quadrupoles just downstream of bump magnet BM3. Calculated results are presented for both scraping scenarios (see Table 9). The average power deposited in the warm components is reasonably small, but the maximum dose in the motionless scraper is extremely high, necessitating its stepwise rotation (Figure 10), as stressed above. Energy deposition in the superconducting quadrupoles in the proposed scheme is well below the tolerable levels.

Table 13. Maximum Energy Deposition Rate $q$, Deposited Power $P$, and Annual Dose $D$ in the Scraper System for "Peak" $\left(1.4 \times 10^{10} \mathrm{p} / \mathrm{s}\right)$ and "Steady State" $\left(1.6 \times 10^{8} \mathrm{p} / \mathrm{s}\right)$ Scraping Rates.

\begin{tabular}{ccc|cc}
\hline \hline Element & \multicolumn{2}{c|}{ Peak } & \multicolumn{2}{c}{ Steady state } \\
& $q(\mathrm{~mW} / \mathrm{g})$ & $P(\mathrm{~W})$ & $P(W)$ & $D(\mathrm{Mrad} / \mathrm{yr})$ \\
\hline SCR & & 14910 & 171.00 & $4.7 \times 10^{7}$ \\
BM2 & 9.18 & 3800.00 & 43.000 & 227 \\
BM3 & 0.004 & 0.45 & 0.005 & 0.100 \\
QU1b & 0.005 & 0.49 & 0.006 & 0.122 \\
QU2b & 0.015 & 1.80 & 0.021 & 0.376 \\
\hline \hline
\end{tabular}

To prevent ground water radioactivation and to reduce the residual activity dose rate, each scraper must be surrounded with an appropriate shield (Figure 10). The overall steel shield length is calculated to be $3 \mathrm{~m}$ and the thickness $d=40 \mathrm{~cm}$ or $75 \mathrm{~cm}$ in order to reduce a gamma dose rate to $100 \mathrm{mrem} / \mathrm{hr}$ or $10 \mathrm{mrem} / \mathrm{hr}$, respectively, and to have no trouble with ground water activation. 


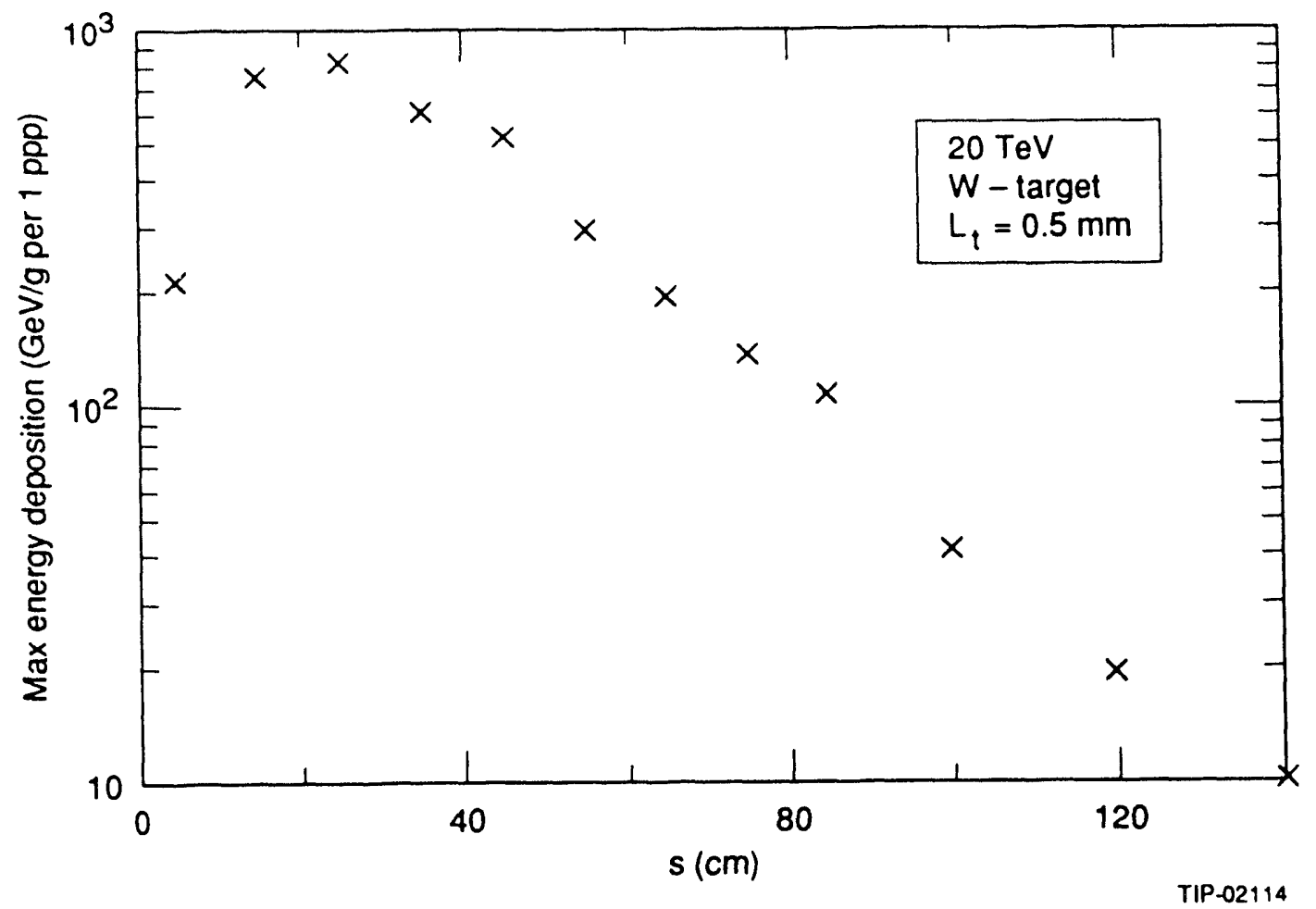

Figure 45. Longitudinal Distribution of Maximum Energy Deposition Density in the Copper Scraper for Chosen Parameters of the Scraper System.

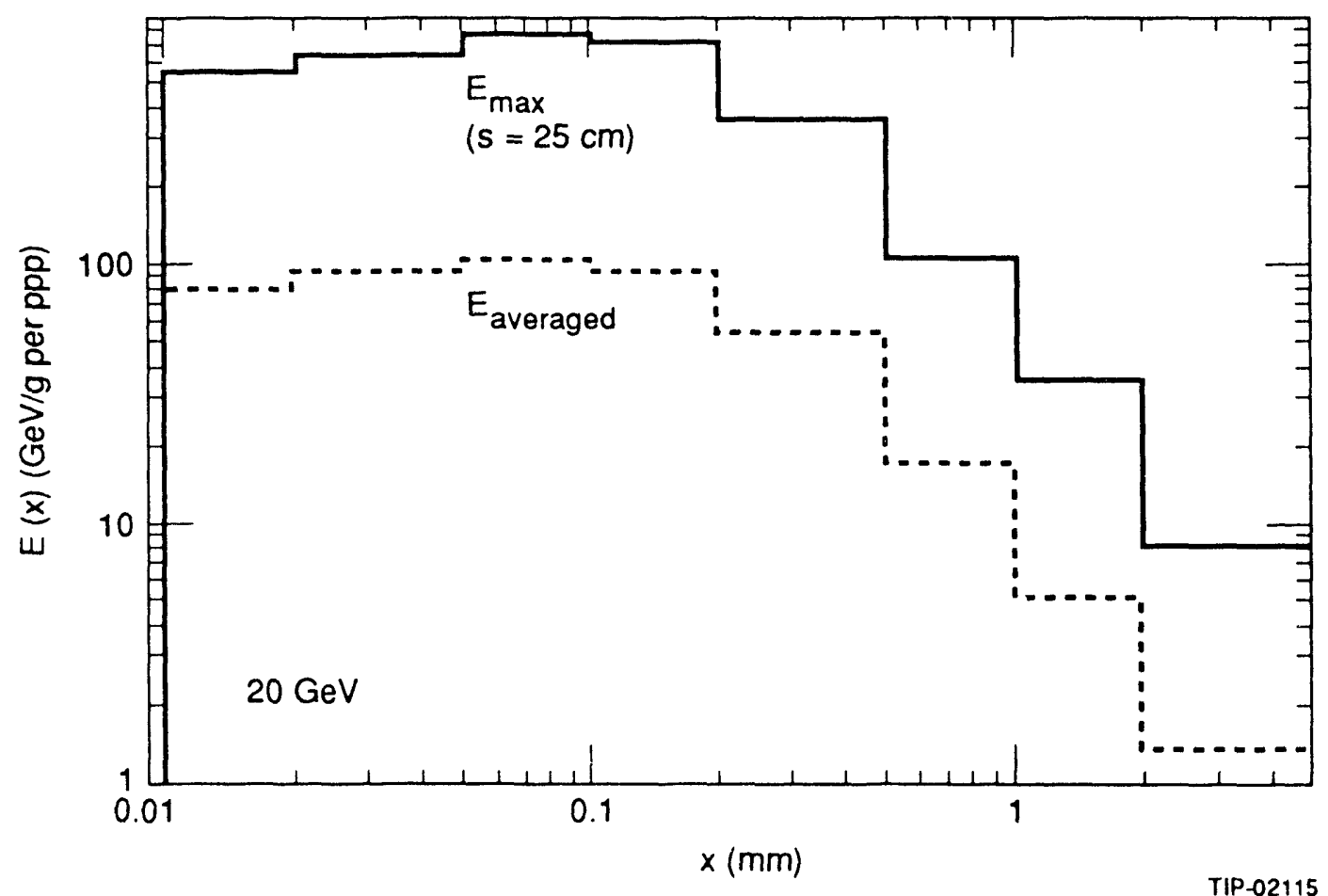

Figure 46. Lateral Distribution of Energy Deposition in the Copper Scraper at Shower Maximum and Averaged Over $120 \mathrm{~cm}$ of Length. 


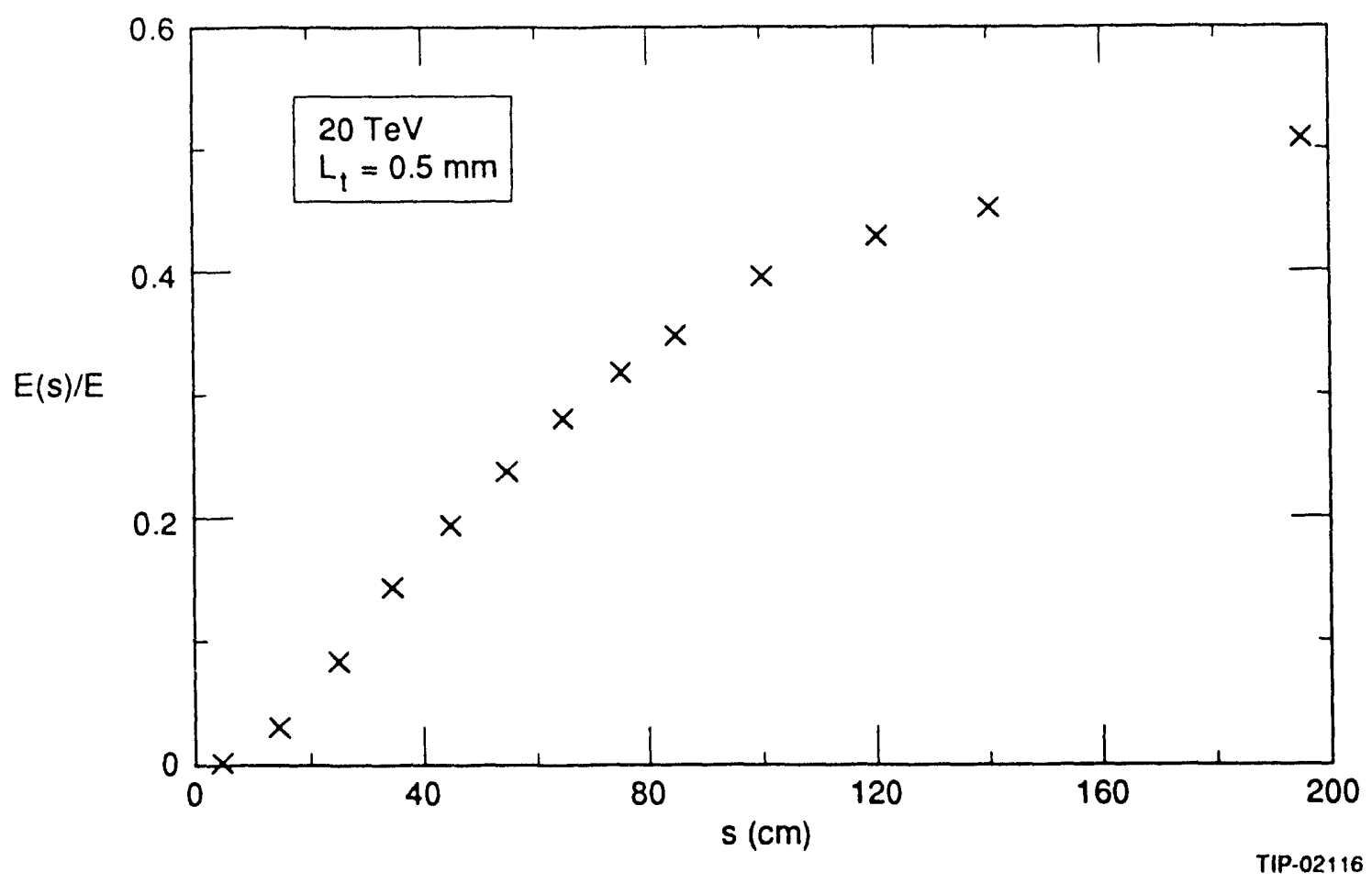

Figure 47. Cumulative Distribution of Energy Absorbed in the Copper Scraper.

\subsection{BEAM HALO IN IP}

As stressed above, the second major goal of the scraper system, after protection of the superconducting magnets, is formation of the transverse emittance required for the physics experiments with a minimal halo at the interaction points and maintenance of this condition throughout the entire collider run. The system proposed in this paper copes with this problem rather well. For the purely diffusion processes, the amplitude increase speed is about $5 \mathrm{\sigma} / \mathrm{s}$ (see Section 5.0), or $5 / 3000 \sigma$ per turn. Inasmuch as the mean lifetime of the halo protons after the first pass through the scraper target is equal to 20-30 turns (Figure 20), this means the maximal halo increase does not exceed $0.05 \sigma / \mathrm{s}$; the rest is intercepted by the scraper.

The results of a numeric simulation of the halo in the interaction region resulting from the scraping of just 30 halo protons are shown in Figures 48-50. The data are presented for the scraper system parameters chosen above with a $0.5-\mathrm{mm}$ tungsten target positioned at $x_{O}=6 \sigma$ of the beam centerline. We see from Figure 50 how efficient the scraping is in removing the halo. It was shown in the previous sections that there are very important advantages to introducing a target in the scraper system. But the amplitude increase due to scatterings in the target results in the spreading of the halo in the region very close to $x_{O}$ (Figure 50). So depending on the experimental requirements (high- or low- $\mathrm{P}_{\mathrm{t}}$ physics), the target-scraper offset can be reduced from 
$50 \mu \mathrm{m}$ up to a few $\mu \mathrm{m}$ or even to zero, after the beam scraping at injection, acceleration and the first $15 \mathrm{~min}$ of collisions. The halo above a certain distance ( $7 \sigma$ in Figure 50 ) is completely removed by controlling the collimators $\mathrm{Cl}-\mathrm{C} 4$.

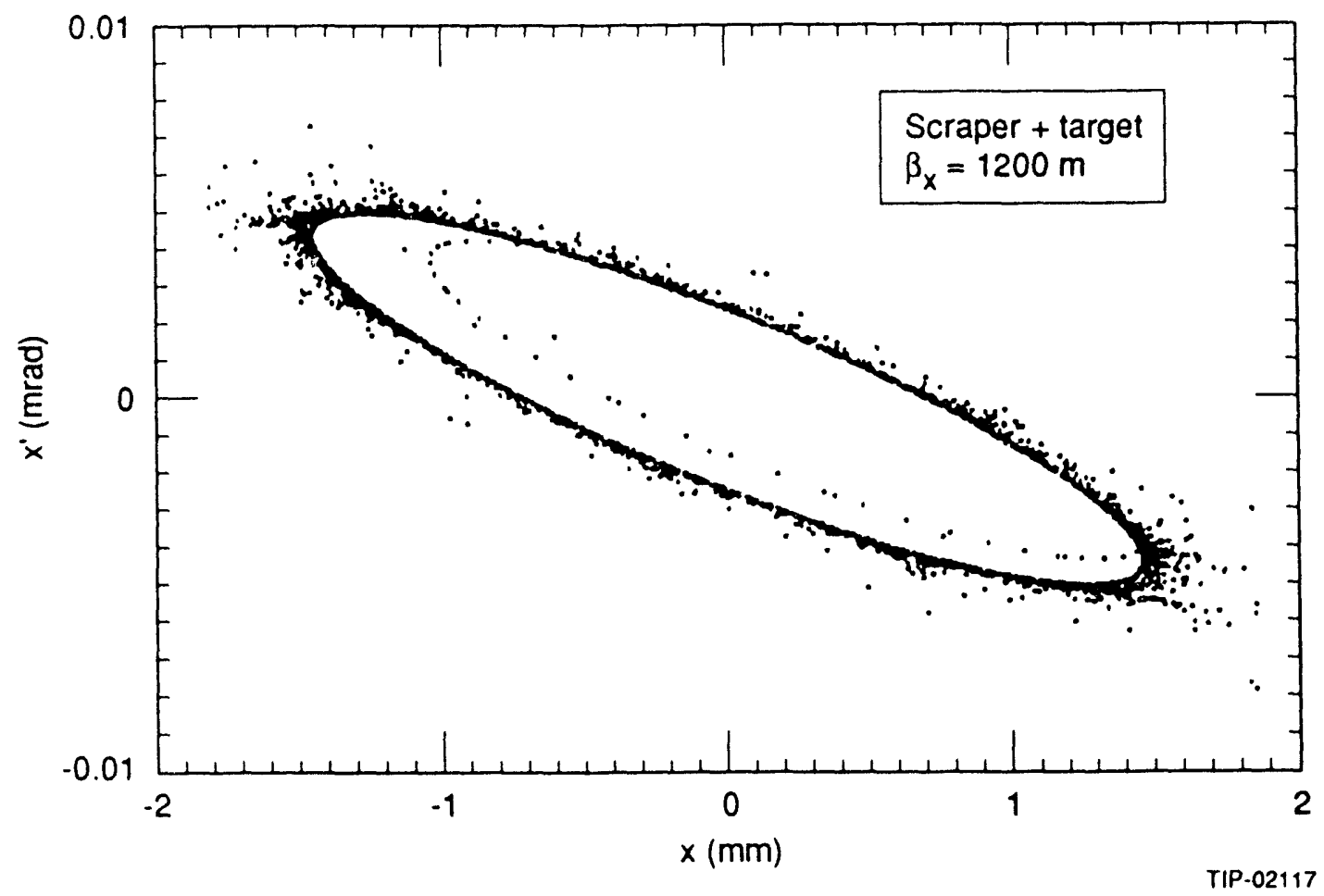

Figure 48. Phase Space Portrait of the Outscattered Protons at the Entrance to the Scraper System. 


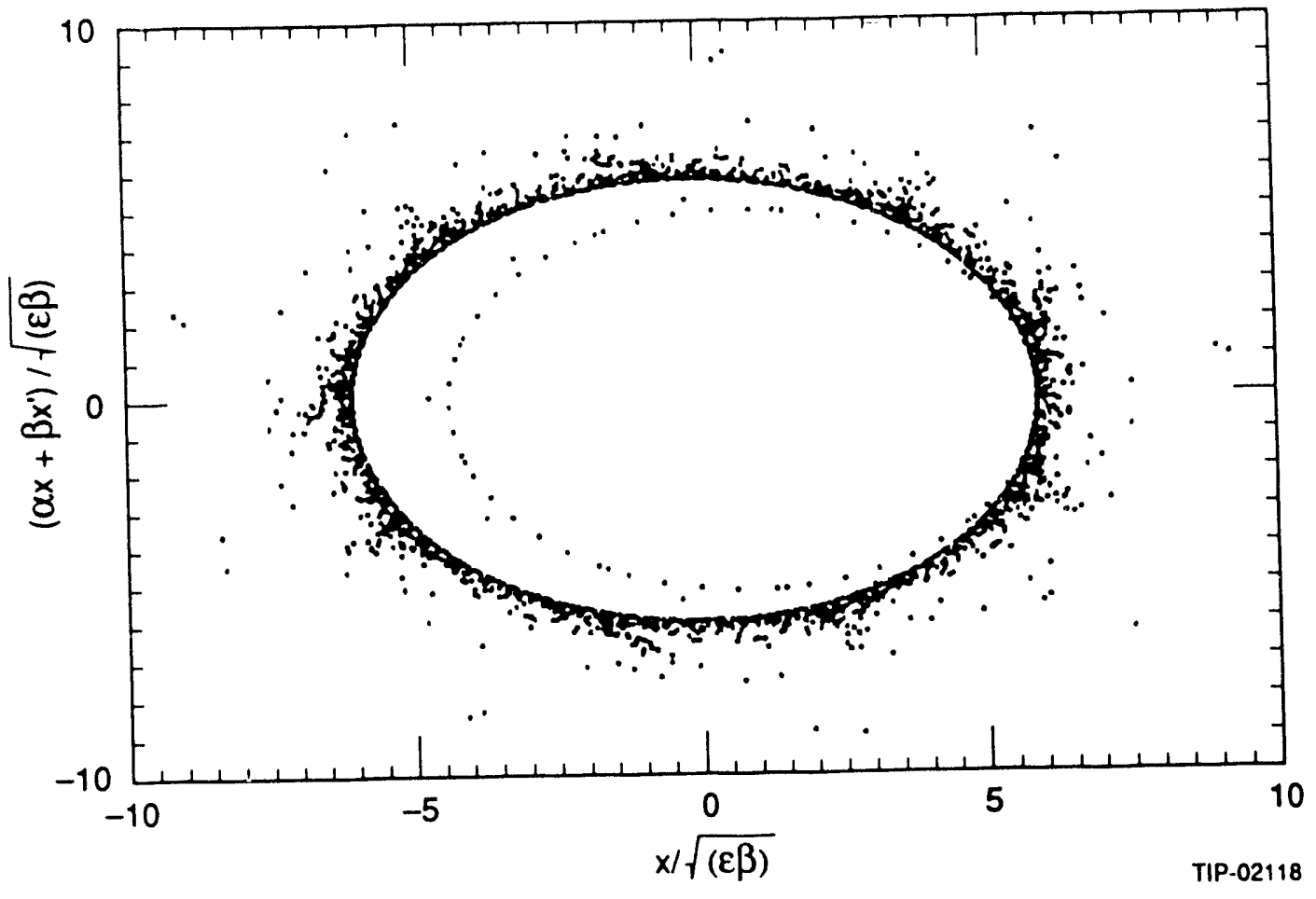

Figure 49. Normalized Phase Space Portrait of Protons Outscattered from the Scraper. 


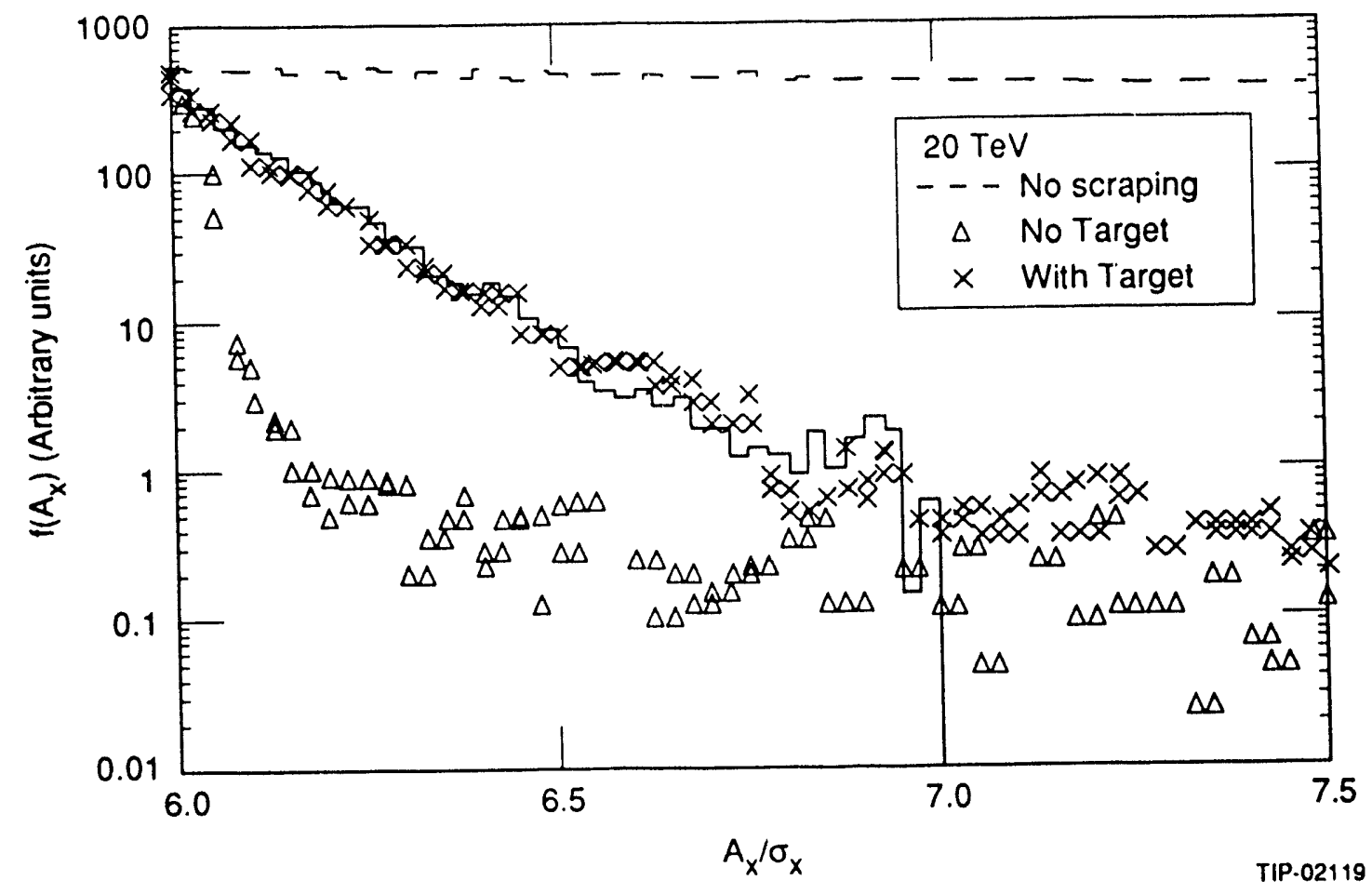

Figure 50. Distribution of Halo Particles With No Scraping, With Single Scraper, With Target/Scraper Couple, and With Collimators $\mathrm{C} 1$ and $\mathrm{C} 3$ Installed Additionally at $7 \sigma$ from the Beam Centerline (histogram). 


\section{ACKNOWLEDGEMENTS}

We are very grateful to Helen Edwards, Dave Ritson, Brett Parker, R. Soundranayagam, and Karen Earley for their useful comments. 


\section{REFERENCES}

1. “SSC Design Report," SSCL-SR-1056, SSC Laboratory (1990).

2. I.S. Baishev, A.I. Drozhdin, and N.V. Mokhov, "Beam Loss and Radiation Effects in the SSC Lattice Elements," SSCL-306, SSC Laboratory (1990).

3. R. Soundranayagam, N.V. Mokhov, M.A. Maslov, and I.A. Yazynin, "The SSC Collider Beam Halo Scraper System," paper presented at Particle Accelerator Conference, San Francisco (1991).

4. Letter of D. Ritson and R. Stiening, SSC Laboratory, March 8, 1991.

5. S. Pruss, Fermilab, private communication.

6. LHC Design Report, Chapter 9, CERN (1991).

7. A. Garren, SYNCH.

8. N.V. Mokhov, Sov. J. Part. Nucl., vol. 18 (5), pp. $408-426$ (1987).

9. A.N. Kalinovsky, N.V. Mokhov, and Yu.P. Nikitin, "Passage of High Energy Particles Through Matter," AIP, New York (1989).

10. I.S. Baishev, M.A. Maslov, and N.V. Mokhov, Proc. VIII All-Union Conference on Charged Particle Accelerators, Dubna, vol. 2, 167 (1983).

11. I.S. Baishev, Preprint IHEP 87-149, Protvino (1987).

12. N.V. Mokhov, "The MARS 10 Code System," FN-509, Fermilab (1989).

13. A.I. Drozhdin, M. Harrison, and N.V. Mokhov, FN-418, Fermilab (1985).

14. A.I. Drozhdin, M.A. Maslov, N.V. Mokhov et al., Proc. X All-Union Conference on Charged Particle Accelerators, Dubna, vol. 2, 278 (1987).

15. M.A. Maslov, N.V. Mokhov, C.T. Murphy, and I.A.Yazynin, SSC Note SSCL-429 (1991). 

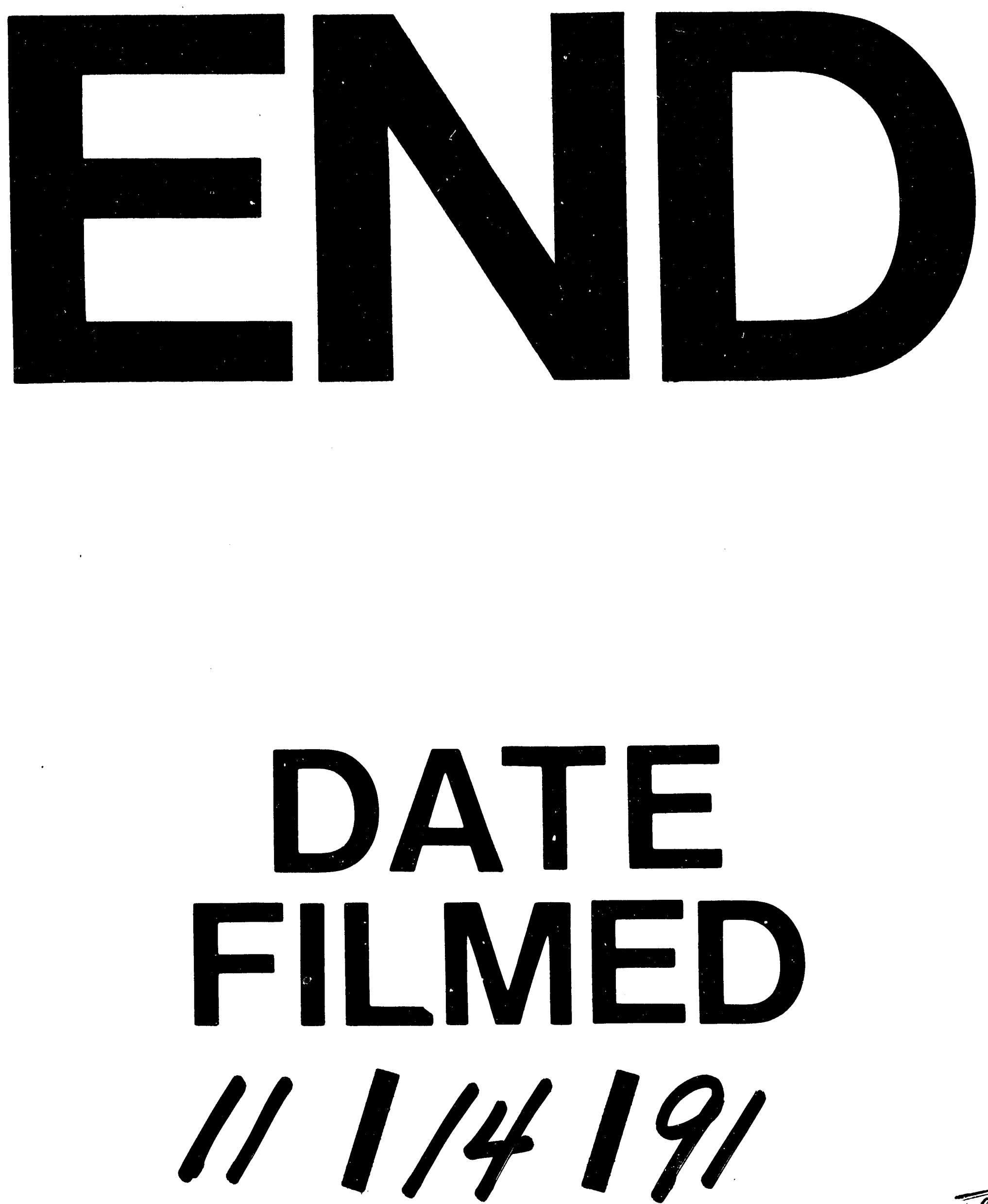

$I_{1}$ 